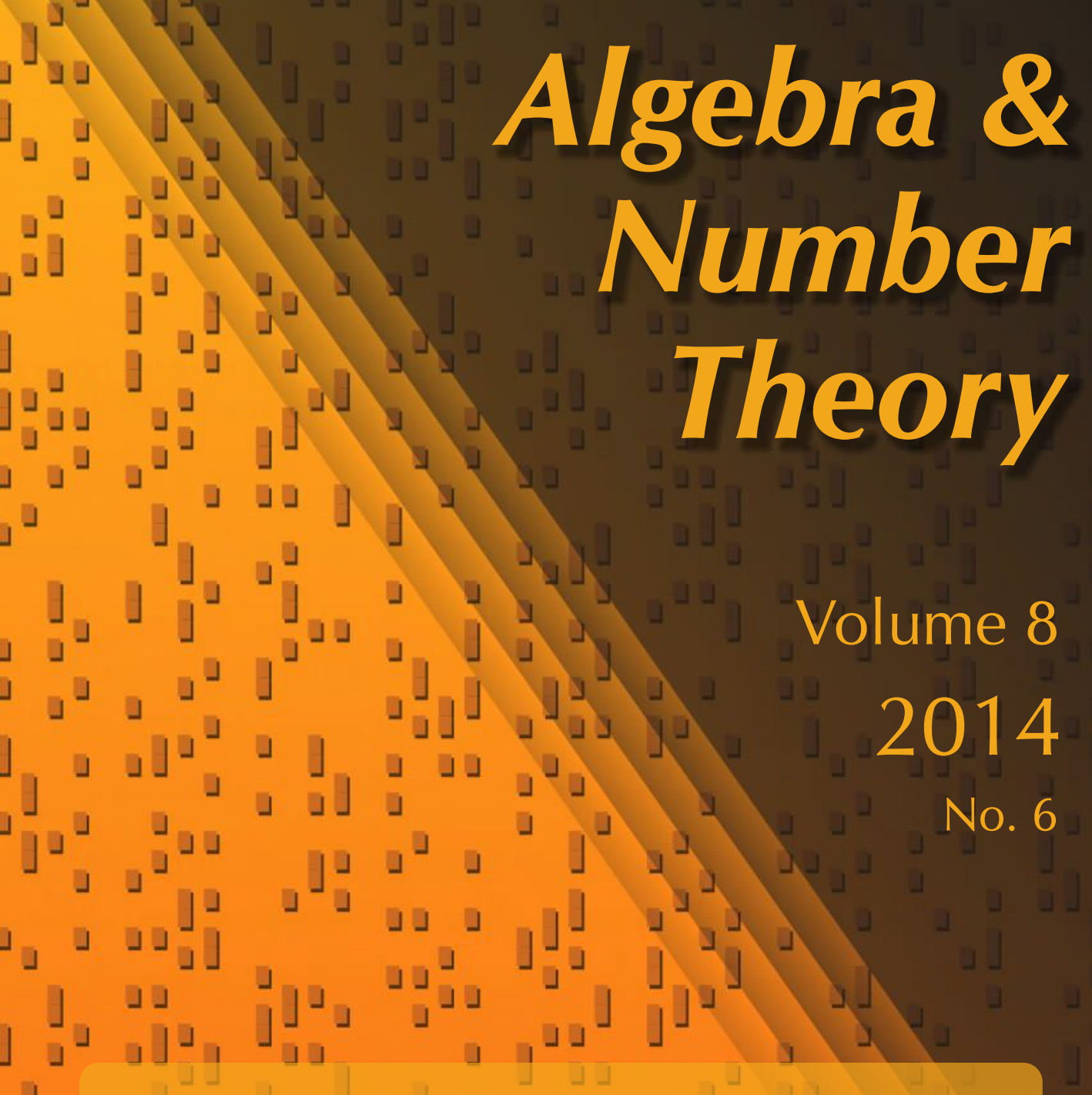




\title{
Locally analytic representations and sheaves on the Bruhat-Tits building
}

\author{
Deepam Patel, Tobias Schmidt and Matthias Strauch
}

Let $L$ be a finite field extension of $\mathbb{Q}_{p}$ and let $G$ be the group of $L$-rational points of a split connected reductive group over $L$. We view $G$ as a locally $L$-analytic group with Lie algebra $\mathfrak{g}$. The purpose of this work is to propose a construction which extends the localization of smooth $G$-representations of P. Schneider and $\mathrm{U}$. Stuhler to the case of locally analytic $G$-representations. We define a functor from admissible locally analytic $G$-representations with prescribed infinitesimal character to a category of equivariant sheaves on the Bruhat-Tits building of $G$. For smooth representations, the corresponding sheaves are closely related to the sheaves of Schneider and Stuhler. The functor is also compatible, in a certain sense, with the localization of $\mathfrak{g}$-modules on the flag variety by A. Beilinson and J. Bernstein.

1. Introduction 1366

2. Distribution algebras and locally analytic representations 1372

3. Completed skew group rings 1378

4. Sheaves on the Bruhat-Tits building and smooth representation $\$ 380$

5. Sheaves on the flag variety and Lie algebra representations 1387

6. Berkovich analytifications 1391

7. A sheaf of "distribution operators" on the building 1404

8. From representations to sheaves 1424

9. Comparison with the Schneider-Stuhler construction 1430

10. Compatibility with the Beilinson-Bernstein localization 1433

11. A class of examples

Appendix: Analyticity of group actions near points on the building 1439

Acknowledgments $\quad 1442$

References 1443

M. Strauch would like to acknowledge the support of the National Science Foundation (award numbers DMS-0902103 and DMS-1202303).

MSC2010: primary 22E50; secondary 20G25, 20G05, 32C38, 11S37, 13N10.

Keywords: locally analytic representations, Bruhat-Tits, buildings, sheaves. 


\section{Introduction}

Let $L$ be a finite field extension of the field $\mathbb{Q}_{p}$ of $p$-adic numbers. Let $\boldsymbol{G}$ be a connected split reductive group over $L$ and $\boldsymbol{B} \subset \boldsymbol{G}$ a Borel subgroup defined over $L$. Let $\boldsymbol{T} \subset \boldsymbol{G}$ be a maximal torus contained in $\boldsymbol{B}$. Let $G:=\boldsymbol{G}(L), T:=\boldsymbol{T}(L)$ denote the groups of rational points, viewed as locally $L$-analytic groups. Let $\mathfrak{g}$ and $t$ be the corresponding Lie algebras.

The purpose of this work is to propose a construction which extends the localization theory for smooth $G$-representations of P. Schneider and U. Stuhler [1997] to the case of locally analytic $G$-representations. In more concrete terms, we define an exact functor from admissible locally analytic $G$-representations with prescribed infinitesimal character to a category of equivariant sheaves on the BruhatTits building of $G$. The functor is also compatible, in a certain sense, with the localization theory for $\mathfrak{g}$-modules on the flag variety of $\boldsymbol{G}$ by A. Beilinson and J. Bernstein [1981], and J.-L. Brylinski and M. Kashiwara [1980; 1981].

To give more details, let $\mathscr{B}$ be the (semisimple) Bruhat-Tits building of $G$. The torus $\boldsymbol{T}$ determines an apartment $A$ in $\mathscr{B}$. We fix a fundamental chamber $\mathscr{C} \subset A$ and a special vertex $x_{0} \in \overline{\mathscr{C}}$, which will be used as an origin for the affine space $A$. In [Schneider and Stuhler 1997] the authors consider, for any facet $F \subset \mathscr{B}$, a well-behaved filtration

$$
P_{F} \supset U_{F}^{(0)} \supset U_{F}^{(1)} \supset \cdots
$$

of the pointwise stabilizer $P_{F}$ of $F$ in $G$ by open pro- $p$ subgroups $U_{F}^{(e)}$. For any point $z \in \mathscr{B}$, one sets $U_{z}^{(e)}:=U_{F}^{(e)}$, where $F$ is the unique facet containing $z$. It forms a fundamental system of neighborhoods of $1 \in P_{z}$, where $P_{z}$ is the stabilizer of $z$. Let from now on $e \geq 0$ be a fixed number (called a level [loc. cit.]).

Using the groups $U_{z}^{(e)}$, Schneider and Stuhler [1997, Section IV] defined an exact functor

$$
V \mapsto \underset{\approx}{V}
$$

from smooth complex $G$-representations to sheaves of complex vector spaces on $\mathscr{B}$. The stalk of the sheaf $\underset{\sim}{V}$ at a point $z$ is given by the coinvariants $V_{U_{z}^{(e)}}$ and the restriction of $\underset{\approx}{V}$ to a facet $F \subset \mathscr{B}$ equals the constant sheaf with fiber $V_{U_{F}^{(e)}}$. The functor $V \mapsto \underset{\approx}{V}$ has particularly good properties when restricted to the subcategory of representations generated by their $U_{x_{0}}^{(e)}$-fixed vectors. It is a major tool in the proof of the Zelevinsky conjecture [loc. cit.].

From now on we fix a complete discretely valued field extension $K$ of $L$. The functor $V \mapsto \underset{\approx}{V}$ can be defined in exactly the same way for smooth $G$-representations on $K$-vector spaces, and produces sheaves of $K$-vector spaces on $\mathscr{B}$. The naive extension of the functor $V \mapsto \underset{\approx}{V}$ to locally analytic representations, by taking coinvariants as above, does not have good properties. For instance, applying this 
procedure to an irreducible finite-dimensional algebraic representation, which is not the trivial representation, produces the zero sheaf. Moreover, if we aim at a picture which is related to the localization theory of $\mathfrak{g}$-modules, then localizing an irreducible algebraic representation should give a line bundle.

We consider the variety of Borel subgroups

$$
X=\boldsymbol{G} / \boldsymbol{B}
$$

of $\boldsymbol{G}$. We let $\mathcal{O}_{X}$ be its structure sheaf and $\mathcal{D}_{X}$ be its sheaf of differential operators. Deriving the left regular action of $\boldsymbol{G}$ on $X$ yields an algebra homomorphism

$$
\alpha: \underline{U}(\mathfrak{g}) \rightarrow \mathcal{D}_{X}
$$

where the source refers to the constant sheaf on $X$ with fiber equal to the universal enveloping algebra $U(\mathfrak{g})$ of $\mathfrak{g}$. Let $Z(\mathfrak{g})$ be the center of the ring $U(\mathfrak{g})$.

The torus $\boldsymbol{T}$ determines a root system. Let $\rho$ be half the sum over the positive roots with respect to $\boldsymbol{B}$. For any algebraic character $\chi-\rho$ of the torus $\boldsymbol{T}$ we have the sheaf $\mathcal{D}_{\chi}$ of differential endomorphisms of the line bundle on $X$ associated with $\chi-\rho$. Any trivialization of the line bundle induces a local isomorphism between $\mathcal{D}_{\chi}$ and $\mathcal{D}_{X}$, and we have $\mathcal{D}_{\rho}=\mathcal{D}_{X}$. More generally, if $\chi-\rho$ is an arbitrary character of $\mathfrak{t}$ there is a sheaf of so-called twisted differential operators $\mathcal{D}_{\chi}$ on $X$. As in the former case, it comes equipped with a morphism $\mathcal{O}_{X} \hookrightarrow \mathcal{D}_{\chi}$ which is locally isomorphic to the canonical morphism $\mathcal{O}_{X} \hookrightarrow \mathcal{D}_{X}$. Moreover, there is an algebra homomorphism $\underline{U}(\mathfrak{g}) \rightarrow \mathcal{D}_{\chi}$ locally isomorphic to $\alpha$. The sheaf $\mathcal{D}_{\chi}$ was first introduced in [Beilinson and Bernstein 1981] as a certain quotient sheaf of the skew tensor product algebra $\mathcal{O}_{X} \# U(\mathfrak{g})$, where we use \# to indicate that the multiplication on the tensor product $\mathcal{O}_{X} \otimes U(\mathfrak{g})$ involves the action of $U(\mathfrak{g})$ on $\mathcal{O}_{X}$.

Let $\chi$ be a character of $\mathfrak{t}$. Let $\theta$ be the character of $Z(\mathfrak{g})$ associated with $\chi$ via the classical Harish-Chandra homomorphism. The above map factors via a homomorphism

$$
\underline{U}(\mathfrak{g})_{\theta} \rightarrow \mathcal{D}_{\chi}
$$

where $U(\mathfrak{g})_{\theta}=U(\mathfrak{g}) \otimes_{Z(\mathfrak{g}), \theta} L$. If $\chi$ is dominant and regular, a version of the localization theorem due to Beilinson and Bernstein asserts that the functor

$$
\Delta_{\chi}: M \mapsto \mathcal{D}_{\chi} \otimes_{\underline{U}(\mathfrak{g})_{\theta}} \underline{M}
$$

is an equivalence of categories between the (left) $U(\mathfrak{g})_{\theta}$-modules and the (left) $\mathcal{D}_{\chi}$-modules which are quasicoherent as $\mathcal{O}_{X}$-modules. The underlined objects refer to the associated constant sheaves on $X$. We remark that a seminal application of this theorem (or rather its complex version) leads to a proof of the Kazhdan-Lusztig multiplicity conjecture [Beilinson and Bernstein 1981; Brylinski and Kashiwara 1980; 1981]. 
The starting point of our work is a result of V. Berkovich [Berkovich 1990; Rémy et al. 2010] according to which the building $\mathscr{B}$ may be viewed as a locally closed subspace

$$
\mathscr{B} \hookrightarrow X^{\text {an }}
$$

of the Berkovich analytification $X^{\text {an }}$ of $X$. This makes it possible to "compare" sheaves on $\mathscr{B}$ and $X^{\text {an }}$ in various ways. Most of what has been said above about the scheme $X$ extends to the analytic space $X^{\text {an }}$. In particular, there is an analytic version $\mathcal{D}_{\chi}^{\text {an }}$ of $\mathcal{D}_{\chi}$ and an analytic version $\Delta_{\chi}(\cdot)^{\text {an }}$ of the functor $\Delta_{\chi}$ (Section 6).

For technical reasons we have to assume at some point in this paper that $L=\mathbb{Q}_{p}$, with $p>2$ an odd prime. (However, we have no doubts that our results eventually extend to general $L$ and $p$ ). To describe our proposed locally analytic "localization functor" under this assumption we let $D(G)$ be the algebra of $K$-valued locally analytic distributions on $G$. It naturally contains $U(\mathfrak{g})$. Recall that the category of admissible locally analytic $G$-representations over $K$ (in the sense of Schneider and J. Teitelbaum [2003]) is antiequivalent to a full abelian subcategory of the (left) $D(G)$-modules, the so-called coadmissible modules. A similar result holds over any compact open subgroup $U_{z}^{(e)}$.

From now on we fix a central character

$$
\theta: Z\left(\mathfrak{g}_{K}\right) \rightarrow K
$$

and a toral character $\chi \in \mathfrak{t}_{K}^{*}$ associated to $\theta$ via the classical Harish-Chandra homomorphism. For example, the character $\chi=\rho$ corresponds to the trivial infinitesimal character $\theta_{0}$ with $\operatorname{ker} \theta_{0}=Z\left(\mathfrak{g}_{K}\right) \cap U\left(\mathfrak{g}_{K}\right) \mathfrak{g}_{K}$. The ring $Z\left(\mathfrak{g}_{K}\right)$ lies in the center of the ring $D(G)$ [Schneider and Teitelbaum 2002, Proposition 3.7], so that we may consider the central reduction

$$
D(G)_{\theta}:=D(G) \otimes_{Z\left(\mathfrak{g}_{K}\right), \theta} K .
$$

We propose to study the abelian category of (left) $D(G)_{\theta}$-modules which are coadmissible over $D(G)$. As remarked above it is antiequivalent to the category of admissible locally analytic $G$-representations over $K$ with infinitesimal character $\theta$. We emphasize that any topologically irreducible admissible locally analytic $G$-representation admits, up to a finite extension of $K$, an infinitesimal character [Dospinescu and Schraen 2013, Corollary 3.10].

To start with, consider a point $z \in \mathscr{B}$. The group $U_{z}^{(e)}$ carries a natural $p$-valuation in the sense of M. Lazard [1965, III.2.1]. According to the general locally analytic theory [Schneider and Teitelbaum 2003, Section 4], this induces a family of norms $\|\cdot\|_{r}$ on the distribution algebra $D\left(U_{z}^{(e)}\right)$ for $r \in\left[r_{0}, 1\right)$, where $r_{0}:=p^{-1}$. We let $D_{r}\left(U_{z}^{(e)}\right)$ be the corresponding completion of $D\left(U_{z}^{(e)}\right)$ and $D_{r}\left(U_{z}^{(e)}\right)_{\theta}$ its central reduction. In Section 8.2 we introduce sheaves of distribution algebras $\underline{D}_{r}$ and $\underline{D}_{r, \theta}$ 
on $\mathscr{B}$ with stalks

$$
\left(\underline{D}_{r}\right)_{z}=D_{r}\left(U_{z}^{(e)}\right), \quad\left(\underline{D}_{r, \theta}\right)_{z}=D_{r}\left(U_{z}^{(e)}\right)_{\theta}
$$

for all points $z \in \mathscr{B}$. The inclusions $U(\mathfrak{g}) \subset D_{r}\left(U_{z}^{(e)}\right)$ sheafify to a morphism $\underline{U}\left(\mathfrak{g}_{K}\right)_{\theta} \rightarrow \underline{D}_{r, \theta}$. Similarly, for any coadmissible $D(G)_{\theta}$-module $M$ we consider a $\underline{D}_{r, \theta}$-module $\underline{M}_{r}$ on $\mathscr{B}$ having stalks

$$
\left(\underline{M}_{r}\right)_{z}=D_{r}\left(U_{z}^{(e)}\right)_{\theta} \otimes_{D\left(U_{z}^{(e)}\right)_{\theta}} M
$$

for all points $z \in \mathscr{B}$. The formation of $\underline{M}_{r}$ is functorial in $M$. The sheaves $\underline{D}_{r, \theta}$, $\underline{M}_{r}$ are constructible and will formally replace the constant sheaves appearing in the definition of the functors $\Delta_{\chi}, \Delta_{\chi}^{\mathrm{an}} \cdot{ }^{1}$

Consider the restriction of the structure sheaf of $X^{\text {an }}$ to $\mathscr{B}$, i.e.,

$$
\mathcal{O}_{\mathscr{B}}=\mathcal{O}_{X} \text { an }\left.\right|_{\mathscr{B}} .
$$

We then define a sheaf of noncommutative rings $\mathscr{D}_{r, \chi}$ on $\mathscr{B}$ which is also a module over $\mathcal{O}_{\mathscr{B}}$ and which is vaguely reminiscent of a "sheaf of twisted differential operators". It has a natural $G$-equivariant structure. It depends on the level $e$, but, following the usage of Schneider and Stuhler [1997, Section IV.1], we suppress this in our notation. More importantly, it depends on the "radius" $r$, which is genuine to the locally analytic situation and is related to a choice of completed distribution algebra $D_{r}\left(U_{z}^{(e)}\right)$ at each point $z \in \mathscr{B}$. Completely analogous to constructing $\mathcal{D}_{\chi}$ out of the skew tensor product algebra $\mathcal{O}_{X} \# U\left(\mathfrak{g}_{K}\right)$ (cf. [Beilinson and Bernstein 1981]) we obtain the sheaf $\mathscr{D}_{r, \chi}$ out of a skew tensor product algebra of the form $\mathcal{O}_{\mathscr{B}} \# \underline{D_{r}}$.

To describe the sheaf $\mathscr{D}_{r, \chi}$ we observe first that, for any point $z \in \mathscr{B}$, the inclusion $U_{z}^{(e)} \subset P_{z}$ implies that there is a locally analytic $U_{z}^{(e)}$-action on the analytic stalk $\mathcal{O}_{\mathscr{B}, z}$. We therefore have the corresponding skew group ring $\mathcal{O}_{\mathscr{B}, z} \# U_{z}^{(e)}$ as well as the skew enveloping algebra $\mathcal{O}_{\mathscr{B}, z} \# U(\mathfrak{g})$, familiar objects from noncommutative ring theory [McConnell and Robson 1987]. In Section 3 and in Sections 6.3 and 6.4, we explain how the completed tensor product

$$
\mathcal{O}_{\mathscr{B}, z} \hat{\otimes}_{L} D_{r}\left(U_{z}^{(e)}\right)
$$

can be endowed with a unique structure of a topological $K$-algebra such that the $\mathcal{O}_{\mathscr{B}, z}$-linear maps

$$
\mathcal{O}_{\mathscr{B}, z} \# U_{z}^{(e)} \rightarrow \mathcal{O}_{\mathscr{B}, z} \hat{\otimes}_{L} D_{r}\left(U_{z}^{(e)}\right), \quad \mathcal{O}_{\mathscr{B}, z} \# U(\mathfrak{g}) \rightarrow \mathcal{O}_{\mathscr{B}, z} \hat{\otimes}_{L} D_{r}\left(U_{z}^{(e)}\right),
$$

${ }^{1} \mathrm{We}$ assume from now on that $e$ is sufficiently large (later in the paper we require $e>e_{\mathrm{st}}$, where $e_{\mathrm{st}}$ is defined in Lemma 6.2.6) and that the radius $r$ is equal to $\sqrt[p]{1 / p}$ for some $m \geq 0$; see Lemma 7.4.7. 
induced by $U_{z}^{(e)} \subset D\left(U_{z}^{(e)}\right)^{\times}$and $U(\mathfrak{g}) \subset D\left(U_{z}^{(e)}\right)$ respectively, become ring homomorphisms. To emphasize this skew multiplication we denote the target of the two maps in (1.1.2) by $\mathcal{O}_{\mathscr{B}, z} \# D_{r}\left(U_{z}^{(e)}\right)$, keeping in mind that there is a completed tensor product involved. This process leads to a sheaf of $K$-algebras $\mathcal{O}_{\mathscr{B}} \# \underline{D}_{r}$ on $\mathscr{B}$ with stalks

$$
\left(\mathcal{O}_{\mathscr{B}} \# \underline{D}_{r}\right)_{z}=\mathcal{O}_{\mathscr{B}, z} \# D_{r}\left(U_{z}^{(e)}\right)
$$

at points $z \in \mathscr{B}$. It comes equipped with a morphism $\mathcal{O}_{\mathscr{B}} \# U(\mathfrak{g}) \rightarrow \mathcal{O}_{\mathscr{B}} \# \underline{D_{r}}$ giving back the second map in (1.1.2) at a point $z \in \mathscr{B}$.

To generalize the formalism of twisting to this new situation we proceed similarly to [Beilinson and Bernstein 1981]. Let $\mathcal{T}_{X}$ an be the tangent sheaf of $X^{\text {an }}$ and let $\alpha^{\text {an }}: \mathfrak{g} \rightarrow \mathcal{T}_{X}$ an be the analytification of the map $\left.\alpha\right|_{\mathfrak{g}}$. There is the sheaf of $L$-Lie algebras

$$
\mathfrak{b}^{\text {o,an }}:=\operatorname{ker}\left(\mathcal{O}_{X} \text { an } \otimes_{L} \mathfrak{g} \stackrel{\alpha^{\text {an }}}{\rightarrow} \mathcal{T}_{X} \text { an }\right) .
$$

The inclusion $\boldsymbol{T} \subset \boldsymbol{B}$ induces an isomorphism of Lie algebras

$$
\mathcal{O}_{X} \text { an } \otimes_{L} \mathfrak{t} \stackrel{\sim}{\longrightarrow} \mathfrak{b}^{\circ, \text { an }} /\left[\mathfrak{b}^{\circ, \text { an }}, \mathfrak{b}^{\circ, \text { an }}\right] .
$$

We have thus an obvious $\mathcal{O}_{X}$ an-linear extension of the character $\chi-\rho$ of $\mathfrak{t}_{K}$ to $\mathfrak{b}^{\circ, \text { an }} \otimes_{L} K$. Its kernel, restricted to the building $\mathscr{B}$, generates a two-sided ideal $\mathscr{I}_{\chi}^{\text {an }}$ in $\mathcal{O}_{\mathscr{B}} \# \underline{D}_{r}$ and we set

$$
\mathscr{D}_{r, \chi}:=\left(\mathcal{O}_{\mathscr{B}} \# \underline{D}_{r}\right) / \mathscr{I}_{\chi}^{\text {an }} .
$$

Let $\mathcal{D}_{\mathscr{B}, \chi}^{\text {an }}$ denote the restriction of $\mathcal{D}_{\chi}^{\text {an }}$ to the building $\mathscr{B}$. The sheaf $\mathscr{D}_{r, \chi}$ comes with an algebra homomorphism $\mathcal{D}_{\mathscr{B}, \chi}^{\text {an }} \rightarrow \mathscr{D}_{r, \chi}$ induced from the inclusion $\mathcal{O}_{\mathscr{B}} \# U\left(\mathfrak{g}_{K}\right) \rightarrow \mathcal{O}_{\mathscr{B}} \# \underline{D}_{r}$. Most importantly, the canonical morphism $\underline{D}_{r} \rightarrow \mathcal{O}_{\mathscr{B}} \# \underline{D}_{r}$ induces a canonical morphism $\underline{D}_{r, \theta} \rightarrow \mathscr{D}_{r, \chi}$ making the diagram

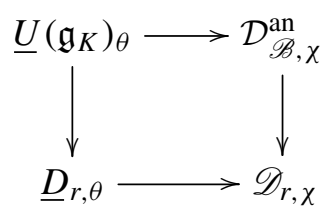

commutative. In this situation we prove that

$$
M \mapsto \mathscr{L}_{r, \chi}(M):=\mathscr{D}_{r, \chi} \otimes_{\underline{D}_{r, \theta}} \underline{M}_{r}
$$

is an exact covariant functor from coadmissible $D(G)_{\theta}$ modules into $G$-equivariant (left) $\mathscr{D}_{r, \chi}$-modules. The stalk of the sheaf $\mathscr{L}_{r, \chi}(M)$ at a point $z \in \mathscr{B}$ with residue field $\kappa(z)$ equals the $(\chi-\rho)$-coinvariants of the $\mathfrak{t}_{K}$-module

$$
\left(\kappa(z) \hat{\otimes}_{L} \underline{M}_{r, z}\right) / \mathfrak{n}_{\pi(z)}\left(\kappa(z) \hat{\otimes}_{L} \underline{M}_{r, z}\right)
$$


as it should [Beilinson and Bernstein 1981]. Here, $\mathfrak{n}_{\pi(z)}$ equals the nilpotent radical of the Borel subalgebra of $\kappa(z) \otimes_{L} \mathfrak{g}$ defined by the point $\pi(z) \in X$, where $\pi: X^{\text {an }} \rightarrow X$ is the canonical map. We tentatively call $\mathscr{L}_{r, \chi}$ a locally analytic "localization functor". We suppress the dependence of $\mathscr{L}_{r, \chi}$ on the level $e$ in our notation.

We prove the following compatibilities with the Schneider-Stuhler and the Beilinson-Bernstein localizations. Suppose first that the coadmissible module $M$ is associated to a smooth $G$-representation $V$. Since $\mathfrak{g} M=0$ it has infinitesimal character $\theta=\theta_{0}$ and the natural choice of twisting is therefore $\chi=\rho$. We establish a canonical isomorphism (Theorem 9.2.5) of $\mathcal{O}_{\mathscr{B}}$-modules

$$
\mathscr{L}_{r_{0}, \rho}(M) \stackrel{\sim}{\rightarrow} \mathcal{O}_{\mathscr{B}} \otimes_{L} \underset{\approx}{\check{V}}
$$

where $\check{V}$ is the smooth dual of $V$ and $\underset{\approx}{\check{V}}$ the sheaf associated to $\check{V}$ by SchneiderStuhler. The isomorphism is natural in $M$.

Secondly, suppose the coadmissible module $M$ is associated to a finite dimensional algebraic $G$-representation. The functor $\Delta_{\chi}(\cdot)^{\text {an }}$ may be applied to its underlying $\mathfrak{g}$-module and gives a $\mathcal{D}_{\chi}^{\text {an }}$-module on $X^{\text {an }}$ and then, via restriction, a $\mathcal{D}_{\mathscr{B}, \chi}^{\mathrm{a}}$-module $\Delta_{\chi}(M)_{\mathscr{B}}^{\mathrm{an}}$ on $\mathscr{B}$. We prove (Theorem 10.1.1) that there is a number $r(M) \in\left[r_{0}, 1\right)$ which is intrinsic to $M$ and a canonical isomorphism of $\mathcal{D}_{\mathscr{B}, \chi}^{\text {an }}$-modules

$$
\mathscr{L}_{r, \chi}(M) \stackrel{\sim}{\longrightarrow} \Delta_{\chi}(M)_{\mathscr{B}}^{\mathrm{an}}
$$

for $r \geq r(M)$. The isomorphism is natural in $M$.

As a class of examples we finally investigate the localizations of locally analytic representations in the image of the functor $\mathcal{F}_{B}^{G}$ introduced by S. Orlik and investigated in [Orlik and Strauch 2010a]. The image of $\mathcal{F}_{B}^{G}$ comprises a wide class of interesting representations and contains all principal series representations as well as all locally algebraic representations (e.g., tensor products of smooth with algebraic representations).

This paper is the first of a series of papers whose aim is to develop a localization theory for locally analytic representations. Here we only make a first step in this direction, focusing on the building and merging the theory of Schneider and Stuhler with the theory of Beilinson and Bernstein, resp. Brylinski and Kashiwara. One approach to get a more complete picture would be to extend the construction given here to a compactification $\overline{\mathscr{B}}$ of the building. The compactification which one would take here is, of course, the closure of $\mathscr{B}$ in $X^{\text {an }}$. Moreover, for intended applications like functorial resolutions and the computation of Ext groups, one has to develop a "homological theory", in analogy to [Schneider and Stuhler 1997, Section II]. However, the sheaves produced in this way (using a compactification) would still have too many global sections. For instance, the space of global sections 
would be a module for the ring of meromorphic functions on $X^{\text {an }}$ with poles outside $\overline{\mathscr{B}}$, and this is a very large ring. The aim would be to produce sheaves whose global sections give back the $D(G)$-module one started with. In [Patel et al. 2013] we explore an approach (in the case of GL(2)) which is based on the use of (a family of) semistable formal models $\mathfrak{X}$ of $X^{\text {an }}$, and we replace $\mathcal{O}_{\mathscr{B}}$ by the pullback of $\mathcal{O}_{\mathfrak{X}} \otimes L$ via the specialization map $X^{\text {an }} \rightarrow \mathfrak{X}$, and the rôle of $\mathscr{D}_{r, \chi}$ is played by arithmetic logarithmic differential operators. In this regard we want to mention related works by C. Noot-Huyghe [2009], and K. Ardakov and S. Wadsley [2013]. While Noot-Huyghe studies localizations of arithmetic $\mathscr{D}$-modules on smooth formal models of $X$, Ardakov and Wadsley define and study localizations of representations of Iwasawa algebras on smooth models. Our present paper is in some sense complementary to these papers, as our focus is on noncompact groups.

Despite the many aspects (like compactifications, homological theory, relation with formal models) that still have to be explored, given the many technical details that one has to take care of we thought it worthwhile to give an account of the constructions as developed up to this point.

Notation. Let $p$ be an odd prime. Let $L / \mathbb{Q}_{p}$ be a finite extension and $K \subseteq \mathbb{C}_{p}$ a complete discretely valued extension of $L$. The absolute value $|\cdot|$ on $\mathbb{C}_{p}$ is normalized by $|p|=p^{-1}$. Let $o_{L} \subset L$ be the ring of integers and $\varpi_{L} \in o_{L}$ a uniformizer. We denote by $v_{L}$ always the normalized $p$-adic valuation on $L$, i.e., $v_{L}(\varpi)=1$. Let $n$ and $e\left(L / \mathbb{Q}_{p}\right)$ be the degree and the ramification index of the extension $L / \mathbb{Q}_{p}$ respectively. Similarly, $o_{K} \subset K$ denotes the integers in $K$ and $\varpi_{K} \in o_{K}$ a uniformizer. Let $k:=o_{K} /\left(\varpi_{K}\right)$ denote the residue field of $K$.

The letter $\boldsymbol{G}$ always denotes a connected reductive linear algebraic group over $L$ which is split over $L$ and $G=\boldsymbol{G}(L)$ denotes its group of rational points.

\section{Distribution algebras and locally analytic representations}

For notions and notation from nonarchimedean functional analysis we refer to [Schneider 2002]. If not indicated otherwise, topological tensor products of locally convex vector spaces are always taken with respect to the projective tensor product topology.

2.1. Distribution algebras. In this section we recall some definitions and results about algebras of distributions attached to locally analytic groups [Schneider and Teitelbaum 2002; 2003]. We consider a locally $L$-analytic group $H$ and denote by $C^{\text {an }}(H, K)$ the locally convex $K$-vector space of locally $L$-analytic functions on $H$ as defined in [Schneider and Teitelbaum 2002]. The strong dual

$$
D(H, K):=C^{\mathrm{an}}(H, K)_{b}^{\prime}
$$


is the algebra of $K$-valued locally analytic distributions on $H$ where the multiplication is given by the usual convolution product. This multiplication is separately continuous. However, if $H$ is compact, then $D(H, K)$ is a $K$-Fréchet algebra. The algebra $D(H, K)$ comes equipped with a continuous $K$-algebra homomorphism

$$
\Delta: D(H, K) \rightarrow D(H, K) \hat{\otimes}_{K, \iota} D(H, K)
$$

which has all the usual properties of a comultiplication [Schneider and Teitelbaum 2005, Section 3 and Appendix]. Here $\iota$ refers to the (complete) inductive tensor product. $^{2}$ If $H$ is compact, then $D(H, K)$ is a Fréchet space and the inductive and projective tensor product topology on the right-hand side coincide [Schneider 2002, 17.6]. Of course, $\Delta\left(\delta_{h}\right)=\delta_{h} \otimes \delta_{h}$ for $h \in H$.

The universal enveloping algebra $U(\mathfrak{h})$ of the Lie algebra $\mathfrak{h}:=\operatorname{Lie}(H)$ of $H$ acts naturally on $C^{\text {an }}(H, K)$. On elements $\mathfrak{x} \in \mathfrak{h}$ this action is given by

$$
(\mathfrak{x} f)(h)=\left.\frac{d}{d t}\left(t \mapsto f\left(\exp _{H}(-t \mathfrak{x}) h\right)\right)\right|_{t=0},
$$

where $\exp _{H}: \mathfrak{h}-\rightarrow H$ denotes the exponential map of $H$, defined in a small neighborhood of 0 in $\mathfrak{h}$. This gives rise to an embedding of $U(\mathfrak{h})_{K}:=U(\mathfrak{h}) \otimes_{L} K$ into $D(H, K)$ via

$$
U(\mathfrak{h})_{K} \hookrightarrow D(H, K), \quad \mathfrak{x} \mapsto(f \mapsto(\mathfrak{x} f)(1)) .
$$

Here $\mathfrak{x} \mapsto \mathfrak{x}$ is the unique antiautomorphism of the $K$-algebra $U(\mathfrak{h})_{K}$ that induces multiplication by -1 on $\mathfrak{h}$. The comultiplication $\Delta$ restricted to $U(\mathfrak{g})_{K}$ gives the usual comultiplication of the Hopf algebra $U(\mathfrak{g})_{K}$, i.e., $\Delta(\mathfrak{x})=\mathfrak{x} \otimes 1+1 \otimes \mathfrak{x}$ for all $\mathfrak{x} \in \mathfrak{h}$.

\subsection{Norms and completions of distribution algebras.}

2.2.1. $p$-valuations. Let $H$ be a compact locally $\mathbb{Q}_{p}$-analytic group. Recall (see [Lazard 1965]) that a $p$-valuation $\omega$ on $H$ is a real-valued function $\omega: H \backslash\{1\} \rightarrow$ $(1 /(p-1), \infty) \subset \mathbb{R}$ satisfying

(i) $\omega\left(g h^{-1}\right) \geq \min (\omega(g), \omega(h))$,

(ii) $\omega\left(g^{-1} h^{-1} g h\right) \geq \omega(g)+\omega(h)$,

(iii) $\omega\left(g^{p}\right)=\omega(g)+1$,

for all $g, h \in H$. As usual one puts $\omega(1):=\infty$ and interprets the above inequalities in the obvious sense if a term $\omega(1)$ occurs.

Let $\omega$ be a $p$-valuation on $H$. It follows from [loc. cit., III.3.1.3/7/9] that the topology on $H$ is defined by $\omega$ [loc. cit., II.1.1.5] and $H$ is a pro- $p$ group. Moreover,

${ }^{2}$ This is the only exception to our general convention to only consider the projective tensor product topology. 
there is a topological generating system $h_{1}, \ldots, h_{d}$ of $H$ such that the map

$$
\mathbb{Z}_{p}^{d} \rightarrow H, \quad\left(a_{1}, \ldots, a_{d}\right) \mapsto h_{1}^{a_{1}} \cdots h_{d}^{a_{d}}
$$

is well-defined and a homeomorphism. Moreover,

$$
\omega\left(h_{1}^{a_{1}} \ldots h_{d}^{a_{d}}\right)=\min \left\{\omega\left(h_{i}\right)+v_{p}\left(a_{i}\right) \mid i=1, \ldots, d\right\},
$$

where $v_{p}$ denotes the $p$-adic valuation on $\mathbb{Z}_{p}$. The sequence $\left(h_{1}, \ldots, h_{d}\right)$ is called a $p$-basis (or an ordered basis; see [Schneider and Teitelbaum 2003, Section 4]) of the $p$-valued group $(H, \omega)$.

Finally, a $p$-valued group $(H, \omega)$ is called $p$-saturated if any $g \in H$ such that $\omega(g)>p /(p-1)$ is a $p$-th power in $H$.

2.2.2. The canonical p-valuation on uniform pro-p groups. We recall some definitions and results about pro- $p$ groups [Dixon et al. 1999, Chapters 3 and 4] in the case $p \neq 2$. In this subsection $H$ will be a pro- $p$ group which is equipped with its topology of a profinite group. Then $H$ is called powerful if $H / H^{p}$ is abelian. Here, $H^{p}$ is the closure of the subgroup generated by the $p$-th powers of its elements. If $H$ is topologically finitely generated one can show that the subgroup $H^{p}$ is open and hence automatically closed. The lower $p$-series $\left(P_{i}(H)\right)_{i \geq 1}$ of an arbitrary pro- $p$ group $H$ is defined inductively by

$$
P_{1}(H):=H, \quad P_{i+1}(H):=\overline{P_{i}(H)^{p}\left[P_{i}(H), H\right]} .
$$

If $H$ is topologically finitely generated, then the groups $P_{i}(H)$ are all open in $H$ and form a fundamental system of neighborhoods of 1 [loc. cit., Proposition 1.16]. A pro- $p$ group $H$ is called uniform if it is topologically finitely generated, powerful and its lower $p$-series satisfies $\left(H: P_{2}(H)\right)=\left(P_{i}(H): P_{i+1}(H)\right)$ for all $i \geq 1$. If $H$ is a topologically finitely generated powerful pro- $p$ group then $P_{i}(H)$ is a uniform pro- $p$ group for all sufficiently large $i$ [loc. cit., 4.2]. Moreover, any compact $\mathbb{Q}_{p}$-analytic group contains an open normal uniform pro- $p$ subgroup [loc. cit., 8.34]. According to [loc. cit., Theorem 9.10], any uniform pro- $p$ group $H$ determines a powerful $\mathbb{Z}_{p^{-}}$ Lie algebra $\mathcal{L}(H){ }^{3}$ Now let $H$ be a uniform pro- $p$ group. It carries a distinguished $p$-valuation $\omega^{\text {can }}$ which is associated to the lower $p$-series and which we call the canonical $p$-valuation. For $h \neq 1$, it is defined by $\omega^{\text {can }}(h)=\max \left\{i \geq 1: h \in P_{i}(H)\right\}$.

2.2.3. Norms arising from $p$-valuations. In this section we let $H$ be a compact $\mathbb{Q}_{p}$-analytic group endowed with a $p$-valuation $\omega$ that has rational values. For convenience of the reader we briefly recall [Schneider and Teitelbaum 2003, Section 4] the construction of a suitable family of submultiplicative norms $\|\cdot\|_{r}, r \in[1 / p, 1)$ on the algebra $D(H, K)$.

\footnotetext{
${ }^{3}$ The adjective powerful refers here to the property $[\mathcal{L}(H), \mathcal{L}(H)] \subseteq p \mathcal{L}(H)$.
} 
Let $h_{1}, \ldots, h_{d}$ be an ordered basis for $(H, \omega)$. The homeomorphism $\psi: \mathbb{Z}_{p}^{d} \simeq H$ given by $\left(a_{1}, \ldots, a_{d}\right) \mapsto h_{1}^{a_{1}} \cdots h_{d}^{a_{d}}$ is a global chart for the $\mathbb{Q}_{p}$-analytic manifold $H$. By functoriality of $C^{\text {an }}(\cdot, K)$ it induces an isomorphism

$$
\psi^{*}: C^{\mathrm{an}}(H, K) \stackrel{\sim}{\longrightarrow} C^{\mathrm{an}}\left(\mathbb{Z}_{p}^{d}, K\right)
$$

of topological $K$-vector spaces. Using Mahler expansions [Lazard 1965, III.1.2.4] we may express elements of $C\left(\mathbb{Z}_{p}^{d}, K\right)$, the space of continuous $K$-valued functions on $\mathbb{Z}_{p}^{d}$, as series $f(x)=\sum_{\alpha \in \mathbb{N}_{0}^{d}} c_{\alpha}\left(\begin{array}{l}x \\ \alpha\end{array}\right)$, where $c_{\alpha} \in K$ and $\left(\begin{array}{l}x \\ \alpha\end{array}\right)=\left(\begin{array}{l}x_{1} \\ \alpha_{1}\end{array}\right) \cdots\left(\begin{array}{l}x_{d} \\ \alpha_{d}\end{array}\right)$ for $x=\left(x_{1}, \ldots, x_{d}\right) \in \mathbb{Z}_{p}^{d}$ and multi-indices $\alpha=\left(\alpha_{1}, \ldots, \alpha_{d}\right) \in \mathbb{N}_{0}^{d}$. Further, we have $\left|c_{\alpha}\right| \rightarrow 0$ as $|\alpha|=\alpha_{1}+\cdots+\alpha_{d} \rightarrow \infty$. A continuous function $f \in C\left(\mathbb{Z}_{p}^{d}, K\right)$ is locally analytic if and only if $\left|c_{\alpha}\right| r^{|\alpha|} \rightarrow 0$ for some real number $r>1$ [loc. cit., III.1.3.9].

Put $b_{i}:=h_{i}-1 \in \mathbb{Z}[H]$ and $\boldsymbol{b}^{\alpha}:=b_{1}^{\alpha_{1}} \cdots b_{d}^{\alpha_{d}}$ for $\alpha \in \mathbb{N}_{0}^{d}$. Identifying group elements with Dirac distributions induces a $K$-algebra embedding $K[H] \hookrightarrow D(H, K)$, $h \mapsto \delta_{h}$. In the light of the dual isomorphism $\psi_{*}: D\left(\mathbb{Z}_{p}^{d}, K\right) \stackrel{\sim}{\rightarrow} D(H, K)$ we see that any $\delta \in D(H, K)$ has a unique convergent expansion $\delta=\sum_{\alpha \in \mathbb{N}_{0}^{d}} d_{\alpha} b^{\alpha}$ with $d_{\alpha} \in K$ such that the set $\left\{\left|d_{\alpha}\right| r^{|\alpha|}\right\}_{\alpha}$ is bounded for all $0<r<1$. Conversely, any such series is convergent in $D(H, K)$. By construction the value $\delta(f) \in K$ of such a series on a function $f \in C^{\text {an }}(H, K)$ equals $\delta(f)=\sum_{\alpha} d_{\alpha} c_{\alpha}$, where $c_{\alpha}$ are the Mahler coefficients of $\psi^{*}(f)$.

To take the original $p$-valuation $\omega$ into account we define $\tau \alpha:=\sum_{i} \omega\left(h_{i}\right) \alpha_{i}$ for $\alpha \in \mathbb{N}_{0}^{d}$. The family of norms $\|\cdot\|_{r}, 0<r<1$, on $D(H, K)$ defined on a series $\delta$ as above via $\|\delta\|_{r}:=\sup _{\alpha}\left|d_{\alpha}\right| r^{\tau \alpha}$ defines the Fréchet topology on $D(H, K)$. Let $D_{r}(H, K)$ denote the norm completion of $D(H, K)$ with respect to $\|\cdot\|_{r}$. Thus we obtain

$$
D_{r}(H, K)=\left\{\sum_{\alpha \in \mathbb{N}_{0}^{d}} d_{\alpha} b^{\alpha}\left|d_{\alpha} \in K, \lim _{|\alpha| \rightarrow \infty}\right| d_{\alpha} \mid r^{\tau \alpha}=0\right\} .
$$

There is an obvious norm-decreasing linear map $D_{r^{\prime}}(H, K) \rightarrow D_{r}(H, K)$ whenever $r \leq r^{\prime}$.

The norms $\|\cdot\|_{r}$ belonging to the subfamily $\frac{1}{p} \leq r<1$ are submultiplicative [loc. cit., Proposition 4.2] and do not depend on the choice of ordered basis [loc. cit., before Theorem 4.11]. In particular, each $D_{r}(H, K)$ is a $K$-Banach algebra in this case. If we equip the projective $\operatorname{limit}_{\lim _{r}} D_{r}(H, K)$ with the projective limit topology the natural map

$$
D(H, K) \stackrel{\sim}{\longrightarrow} \lim _{r} D_{r}(H, K)
$$

is an isomorphism of topological $K$-algebras. Finally, it is easy to see that the comultiplication $\Delta$ completes to continuous "comultiplications" 


$$
\Delta_{r}: D_{r}(H, K) \rightarrow D_{r}(H, K) \hat{\otimes}_{K} D_{r}(H, K)
$$

for any $r$ in the above range. We make two final remarks in case $H$ is a uniform pro- $p$ group and $\omega$ is its canonical $p$-valuation; see Section 2.2.2. In this case each group $P_{m}(H), m \geq 0$ is a uniform pro- $p$ group.

(i) For $r=\frac{1}{p}$ there is a canonical isomorphism between $D_{1 / p}\left(H, \mathbb{Q}_{p}\right)$ and the $p$-adic completion (with $p$ inverted) of the universal enveloping algebra of the $\mathbb{Z}_{p}$-Lie algebra $\frac{1}{p} \mathcal{L}(H)$ [Ardakov and Wadsley 2013, Theorem 10.4, Remark 10.5(c)].

(ii) Let

$$
r_{m}:=\sqrt[p]{1 / p}
$$

for $m \geq 0$. In particular, $r_{0}=1 / p$. Since $P_{m+1}(H)$ is uniform pro- $p$ we may consider the corresponding $\|\cdot\|_{r_{0}}$ - norm on its distribution algebra $D\left(P_{m+1}(H)\right)$. In this situation the ring extension $D\left(P_{m+1}(H)\right) \subset D(H)$ completes in the $\|\cdot\|_{r_{m}}$-norm topology on $D(H)$ to a ring extension

$$
D_{r_{0}}\left(P_{m+1}(H)\right) \subset D_{r_{m}}(H)
$$

and $D_{r_{m}}(H)$ is a finite and free (left or right) module over $\left.D_{r_{0}}\left(P_{m+1}(H)\right)\right)$ with basis given by any system of coset representatives for the finite group $H / P_{m+1}(H)$ [Schmidt 2013, Lemma 5.11].

2.3. Coadmissible modules. We keep all notations from the preceding section but suppose that the $p$-valuation $\omega$ on $H$ satisfies additionally

(HYP) $(H, \omega)$ is $p$-saturated and the ordered basis $h_{1}, \ldots, h_{d}$ of $H$ satisfies $\omega\left(h_{i}\right)+\omega\left(h_{j}\right)>p /(p-1)$ for any $1 \leq i \neq j \leq d$.

Remark. This implies that $H$ is a uniform pro- $p$ group. Conversely, the canonical $p$-valuation on a uniform pro- $p$ group ( $p$ arbitrary) satisfies (HYP). For both statements we refer to [Schneider and Teitelbaum 2003, Remark before Lemma 4.4] and [Schmidt 2008, Proposition 2.1].

Suppose in the following $r \in\left(p^{-1}, 1\right)$ and $r \in p^{\mathbb{Q}}$. In this case the norm $\|\cdot\|_{r}$ on $D_{r}(H, K)$ is multiplicative and $D_{r}(H, K)$ is a (left and right) noetherian integral domain [Schneider and Teitelbaum 2003, Theorem 4.5]. For two numbers $r \leq r^{\prime}$ in the given range the ring homomorphism

$$
D_{r^{\prime}}(H, K) \rightarrow D_{r}(H, K)
$$

makes the target a flat (left or right) module over the source [Schneider and Teitelbaum 2003, Theorem 4.9]. The above isomorphism $D(H, K) \stackrel{\sim}{\longrightarrow} \lim _{r} D_{r}(H, K)$ realizes therefore a Fréchet-Stein structure on $D(H, K)$ in the sense of [loc. cit., Section 3]. The latter allows one to define a well-behaved abelian full subcategory $\mathcal{C}_{H}$ 
of the (left) $D(H, K)$-modules, the so-called coadmissible modules. By definition, an abstract (left) $D(H, K)$-module $M$ is coadmissible if for all $r$ in the given range

(i) $M_{r}:=D_{r}(H, K) \otimes_{D(H, K)} M$ is finitely generated over $D_{r}(H, K)$,

(ii) the natural map $M \stackrel{\sim}{\longrightarrow} \lim _{r} M_{r}$ is an isomorphism.

The projective system $\left\{M_{r}\right\}_{r}$ is sometimes called the coherent sheaf associated to $M$. To give an example, any finitely presented $D(H, K)$-module is coadmissible.

More generally, for any compact locally $L$-analytic group $H$ the $\operatorname{ring} D(H, K)$ has the structure of a Fréchet-Stein algebra [loc. cit., Theorem 5.1]. In particular, we may define the notion of a coadmissible module over $D(H, K)$ for any compact $L$-analytic group in a similar manner. For a general locally $L$-analytic group $G$, a $D(G, K)$-module $M$ is coadmissible if it is coadmissible as a $D(H, K)$-module for every compact open subgroup $H \subset G$. It follows from [loc. cit.] that it is sufficient to check this for a single compact open subgroup.

2.4. Locally analytic representations. A topological abelian group $M$ which is a (left) module over a topological ring $R$ is a separately continuous (left) module, if the map $R \times M \rightarrow M$ giving the action is separately continuous. Any separately continuous bilinear map between Fréchet spaces is jointly continuous [Bourbaki 1987, III.30, Corollary 1].

After this preliminary remark, we recall some facts about locally analytic representations. A $K$-vector space $V$ which equals a locally convex inductive limit $V=\lim _{\rightarrow} \in \mathbb{N} V_{n}$ over a countable system of $K$-Banach spaces $V_{n}$, where the transition maps $\vec{V}_{n}^{n \in \mathbb{N}} \rightarrow V_{n+1}$ are injective compact linear maps is called a vector space of compact type. We recall that such a space is Hausdorff, complete, bornological and reflexive [Schneider and Teitelbaum 2002, Theorem 1.1]. Its strong dual $V_{b}^{\prime}$ is a nuclear Fréchet space satisfying $V_{b}^{\prime}=\lim _{\leftarrow}\left(V_{n}\right)_{b}^{\prime}$. We will make frequent use of the following property of such spaces.

Proposition 2.4.1. Let $W$ be a $K$-Banach space. The continuous linear map

$$
\pi: \underline{\lim } V_{n} \hat{\otimes}_{K} W \rightarrow\left(\underline{\lim } V_{n}\right) \hat{\otimes}_{K} W
$$

is bijective and the source of $\pi$ is Hausdorff. Here, the target of $\pi$ equals the Hausdorff completion of the projective tensor product $\left(\stackrel{\left.\lim V_{n}\right)}{\rightarrow} \otimes_{K} W\right.$.

Proof. The first assertion follows from [Schneider and Teitelbaum 2002, Proposition 1.5] together with [Schneider 2002, Corollary 18.8]. Since the target of $\pi$ is Hausdorff, the second assertion follows from [Schneider 2002, 4.6].

Now let $H$ be a locally $L$-analytic group, $V$ a Hausdorff locally convex $K$-vector space and $\rho: H \rightarrow \operatorname{GL}(V)$ a homomorphism. Then $V$ (or the pair $(V, \rho))$ is called a locally analytic representation of $H$ if the topological $K$-vector space $V$ is barrelled, 
each $h \in H$ acts $K$-linearly and continuously on $V$, and the orbit maps $\rho_{v}: H \rightarrow V$, $h \mapsto \rho(h)(v)$ are locally analytic maps for all $v \in V$ [Schneider and Teitelbaum 2002, Section 3]. If $V$ is of compact type, then the contragredient $H$-action on its strong dual $V_{b}^{\prime}$ extends to a separately continuous left $D(H, K)$-module on a nuclear Fréchet space.

In this way the functor $V \mapsto V_{b}^{\prime}$ induces an antiequivalence of categories between locally analytic $H$-representations on $K$-vector spaces of compact type (with continuous linear $H$-maps as morphisms) and separately continuous $D(H, K)$-modules on nuclear Fréchet spaces (with continuous $D(H, K)$-module maps as morphisms).

A locally analytic $H$-representation $V$ is said to be admissible if $V_{b}^{\prime}$ is a coadmissible $D(H, K)$-module. The above functor restricts to an antiequivalence between the corresponding categories of admissible locally analytic representations and coadmissible $D(H, K)$-modules.

\section{Completed skew group rings}

In this section we will describe a general method of completing certain skew group rings. We recall our general convention that in this paper we only consider the completed tensor product of locally convex vector spaces with respect to the projective tensor product topology. ${ }^{4}$

3.1. Preliminaries. Let $H$ be a compact locally $L$-analytic group and let $A$ be a locally convex $L$-algebra equipped with a locally analytic $H$-representation $\rho$ : $H \rightarrow \mathrm{GL}(A)$ by automorphisms of $L$-algebras. The $H$-action on $A$ extends to $D(H, L)$ and makes $A$ a separately continuous $D(H, L)$-module [Schneider and Teitelbaum 2002, Proposition 3.2]. On the other hand, $D(H, L)$ is a topological module over itself via left multiplication. The completion $A \hat{\otimes}_{L} D(H, L)$ is thus a separately continuous $D(H, L) \hat{\otimes}_{L} D(H, L)$-module. We view it as a separately continuous $D(H, L)$-module by restricting scalars via the comultiplication $\Delta$. This allows us to define the $L$-bilinear map

$$
\left(A \otimes_{L} D(H, L)\right) \times\left(A \hat{\otimes}_{L} D(H, L)\right) \rightarrow A \hat{\otimes}_{L} D(H, L)
$$

given by $\left(\sum_{i} f_{i} \otimes \delta_{i}, b\right) \mapsto \sum_{i} f_{i} \cdot \delta_{i}(b)$. We consider the product topology on the source. In view of the separate continuity of all operations involved together with [Schneider 2002, Lemma 17.1] this map is separately continuous. Since the target is complete it extends in a bilinear and separately continuous manner to the completion of the source. In other words, $A \hat{\otimes}_{L} D(H, L)$ becomes a separately continuous $L$-algebra. Of course, $A \hat{\otimes}_{L} D(H, K)$ is then a separately continuous $K$-algebra. To emphasize its skew multiplication we denote it in the following

\footnotetext{
${ }^{4}$ The only exception occurred in Section 2.1 .
} 
by

$$
A \#_{L} D(H, K)
$$

or even by $A \# D(H, K)$. This should not cause confusion. However, one has to keep in mind that there is a completed tensor product involved. If $A$ is a Fréchet algebra, then the multiplication on $A \# D(H, K)$ is jointly continuous, i.e., $A \# D(H, K)$ is a topological algebra in the usual sense.

\subsection{Skew group rings, skew enveloping algebras and their completions.}

3.2.1. Using the action $\rho$ we may form the abstract skew group ring $A \# H$ [McConnell and Robson 1987, 1.5.4]. We remind the reader that it equals the free left $A$-module with elements of $H$ as a basis and with multiplication defined by $(a g) \cdot(b h):=a(\rho(g)(b)) g h$ for any $a, b \in A$ and $g, h \in H$. Each element of $A \# H$ has a unique expression as $\sum_{h \in H} a_{h} h$ with $a_{h}=0$ for all but finitely many $h \in H$. Evidently, $A \# H$ contains $H$ as a subgroup of its group of units and $A$ as a subring. Furthermore, the inclusion $L[H] \subseteq D(H, L)$ gives rise to an $A$-linear map

$$
A \# H=A \otimes_{L} L[H] \rightarrow A \# D(H, L) .
$$

On the other hand, let $\mathfrak{h}:=\operatorname{Lie}(H)$. Differentiating the locally analytic action $\rho$ gives a homomorphism of $L$-Lie algebras $\alpha: \mathfrak{h} \rightarrow \operatorname{Der}_{L}(A)$ into the $L$-derivations of the algebra $A$ making the diagram

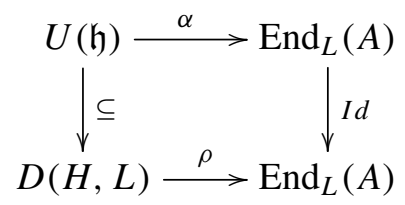

commutative [Schneider and Teitelbaum 2002, 3.1]. We may therefore form the skew enveloping algebra $A \# U(\mathfrak{h})$ [McConnell and Robson 1987, 1.7.10]. We recall that this is an $L$-algebra whose underlying $L$-vector space equals the tensor product $A \otimes_{L} U(\mathfrak{h})$. The multiplication is defined by

$$
\left(f_{1} \otimes \mathfrak{x}_{1}\right) \cdot\left(f_{2} \otimes \mathfrak{x}_{2}\right)=\left(f_{1} \alpha\left(\mathfrak{x}_{1}\right)\left(f_{2}\right)\right) \otimes \mathfrak{x}_{2}+\left(f_{1} f_{2}\right) \otimes\left(\mathfrak{x}_{1} \mathfrak{x}_{2}\right),
$$

for $f_{i} \otimes \mathfrak{x}_{i} \in A \otimes_{L} \mathfrak{h}$. Also, the inclusion $U(\mathfrak{h}) \subseteq D(H, L)$ induces an $A$-linear map

$$
A \# U(\mathfrak{h}) \rightarrow A \# D(H, L) .
$$

Proposition 3.2.4. The A-linear maps (3.2.2) and (3.2.3) are L-algebra homomorphisms. The first of these maps has dense image.

Proof. The first statement follows from the identities

(i) $\left(1 \hat{\otimes} \delta_{g}\right) \cdot(f \hat{\otimes} 1)=(\rho(g)(f)) \hat{\otimes} \delta_{g} \quad$ for any $g \in H, f \in A$,

(ii) $(1 \hat{\otimes} \mathfrak{x}) \cdot(f \hat{\otimes} 1)=(\alpha(\mathfrak{x})(f)) \hat{\otimes} 1+f \hat{\otimes} \mathfrak{x} \quad$ for any $\mathfrak{x} \in \mathfrak{h}, f \in A$ 
in $A \hat{\otimes}_{L} D(H, L)$. In turn these identities follow from $\Delta\left(\delta_{g}\right)=\delta_{g} \hat{\otimes} \delta_{g}$ and $\Delta(\mathfrak{x})=\mathfrak{x} \hat{\otimes} 1+1 \hat{\otimes} \mathfrak{x}$. The final statement follows from [Schneider and Teitelbaum 2002, Lemma 3.1].

3.2.5. In this paragraph we assume that $L=\mathbb{Q}_{p}$ and that the compact locally $\mathbb{Q}_{p^{-}}$ analytic group $H$ is endowed with a $p$-valuation $\omega$. Recall from Section 2.2.3 that $r_{0}:=p^{-1}$. Consider the norm completion $D_{r}(H, L)$ for some arbitrary but fixed $r \in\left[r_{0}, 1\right)$. Let us assume for a moment that the natural map $D(H, L) \rightarrow D_{r}(H, L)$ satisfies the following hypothesis:

( $\star$ ) The separately continuous $D(H, L)$-module structure of $A$ extends to a separately continuous $D_{r}(H, L)$-module structure.

If we replace in the above discussion the comultiplication $\Delta$ by its completion $\Delta_{r}$ we obtain in an entirely analogous manner a completion $A \hat{\otimes}_{L} D_{r}(H, K)$ of the skew group ring $A \# H$, base changed to $K$. It satisfies mutatis mutandis the statement of the preceding proposition. As before we will often abbreviate it by $A \# D_{r}(H, K)$.

\section{Sheaves on the Bruhat-Tits building and smooth representations}

\subsection{Filtrations of stabilizer subgroups.}

4.1.1. Let $\boldsymbol{T}$ be a maximal $L$-split torus in $\boldsymbol{G}$. Let $X^{*}(\boldsymbol{T})$ resp. $X_{*}(\boldsymbol{T})$ be the group of algebraic characters resp. cocharacters of $\boldsymbol{T}$. Let $\Phi=\Phi(\boldsymbol{G}, \boldsymbol{T}) \subset X^{*}(\boldsymbol{T})$ denote the root system determined by the adjoint action of $\boldsymbol{T}$ on the Lie algebra of $\boldsymbol{G}$. Let $W$ denote the corresponding Weyl group. For each $\alpha \in \Phi$ we have the unipotent root subgroup $\boldsymbol{U}_{\alpha} \subseteq \boldsymbol{G}$. Since $\boldsymbol{G}$ is split the choice of a Chevalley basis determines a system of $L$-group isomorphisms

$$
x_{\alpha}: \mathbb{G}_{a} \stackrel{\sim}{\longrightarrow} \boldsymbol{U}_{\alpha}
$$

for each $\alpha \in \Phi$ (an épinglage) satisfying Chevalley's commutation relations [1955, p. 27]. Let $X_{*}(\boldsymbol{C})$ denote the group of $L$-algebraic cocharacters of the connected center $\boldsymbol{C}$ of $\boldsymbol{G}$.

We denote by $G, T, U_{\alpha}$ the groups of $L$-rational points of $\boldsymbol{G}, \boldsymbol{T}, \boldsymbol{U}_{\alpha}(\alpha \in \Phi)$ respectively. Recall the normalized $p$-adic valuation $v_{L}$ on $L$, i.e., $v_{L}(\varpi)=1$. For $\alpha \in \Phi$ we denote by $\left(U_{\alpha, r}\right)_{r \in \mathbb{R}}$ the filtration of $U_{\alpha}$ arising from the valuation $v_{L}$ on $L$ via the isomorphism $x_{\alpha}$. It is an exhaustive and separated discrete filtration by subgroups. Put $U_{\alpha, \infty}:=\{1\}$.

4.1.2. Let $\mathscr{B}=\mathscr{B}(G)$ be the semisimple Bruhat-Tits building of $G$. The torus $\boldsymbol{T}$ determines an apartment $A$ in $\mathscr{B}$. Recall that a point $z$ in the Coxeter complex $A$ is called special if for any direction of wall there is a wall of $A$ actually passing 
through $z$ [Bruhat and Tits 1972, 1.3.7]. As in [Cartier 1979, 3.5] we choose once and for all a special vertex $x_{0}$ in $A$ and a chamber $\mathscr{C} \subset A$ containing it. We use the point $x_{0}$ to identify the affine space $A$ with the real vector space

$$
A=\left(X_{*}(\boldsymbol{T}) / X_{*}(\boldsymbol{C})\right) \otimes_{\mathbb{Z}} \mathbb{R} .
$$

Each root $\alpha \in \Phi$ induces therefore a linear form $\alpha: A \rightarrow \mathbb{R}$ in an obvious way. For any nonempty subset $\Omega \subseteq A$ we let $f_{\Omega}: \Phi \rightarrow \mathbb{R} \cup\{\infty\}, \alpha \mapsto-\inf _{x \in \Omega} \alpha(x)$. It is a concave function in the sense of [Bruhat and Tits 1972, 6.4.1-5]. We emphasize that the concept of a concave function is developed in [loc. cit.] more generally for functions taking values in the set

$$
\tilde{\mathbb{R}}:=\mathbb{R} \cup\{r+: r \in \mathbb{R}\} \cup\{\infty\} .
$$

The latter naturally has the structure of a totally ordered commutative monoid extending the total order and the addition on $\mathbb{R}$. For any $\alpha \in \Phi$ and $r \in \mathbb{R}$ we define

$$
U_{\alpha, r+}:=\bigcup_{s \in \mathbb{R}, s>r} U_{\alpha, s} .
$$

For any concave function $f: \Phi \rightarrow \tilde{\mathbb{R}}$ we then have the group

$$
U_{f}:=\operatorname{subgroup~of~} G \text { generated by all } U_{\alpha, f(\alpha)} \text { for } \alpha \in \Phi .
$$

4.1.4. For each nonempty subset $\Omega \subseteq \mathscr{B}$ we let

$$
P_{\Omega}:=\{g \in G: g z=z \text { for any } z \in \Omega\}
$$

be its pointwise stabilizer in $G$. For any facet $F \subseteq \mathscr{B}$ we will recall from [Schneider and Stuhler 1997, I.2] a certain decreasing filtration of $P_{F}$ by open normal pro- $p$ subgroups which will be most important for all that follows in this article. To do this we first consider a facet $F$ in the apartment $A$. For $\alpha \in \Phi$ we put $f_{F}^{*}(\alpha):=f_{F}(\alpha)+$ if $\left.\alpha\right|_{F}$ is constant and $f_{F}^{*}(\alpha):=f_{F}(\alpha)$ otherwise. This yields a concave function $f_{F}^{*}: \Phi \rightarrow \tilde{\mathbb{R}}$. With $f_{F}^{*}$ also the functions $f_{F}^{*}+e$, for any integer $e \geq 0$, are concave. Hence there is the descending sequence of subgroups

$$
U_{f_{F}^{*}} \supseteq U_{f_{F}^{*}+1} \supseteq U_{f_{F}^{*}+2} \supseteq \cdots
$$

4.1.5. On the other hand we let $\mathfrak{T}:=\operatorname{Spec}\left(o_{L}\left[X^{*}(\boldsymbol{T})\right]\right)$ and

$$
T^{(e)}:=\operatorname{ker}\left(\mathfrak{T}\left(o_{L}\right) \rightarrow \mathfrak{T}\left(o_{L} / \varpi_{L}^{e+1} o_{L}\right)\right)
$$

for any $e \geq 0$ (see [Schneider and Stuhler 1997, proof of Proposition I.2.6]) and finally define

$$
U_{F}^{(e)}:=U_{f_{F}^{*}+e} \cdot T^{(e)}
$$


for each $e \geq 0$ [loc. cit., p. 21]. This definition is extended to any facet $F$ in $\mathscr{B}$ by putting $U_{F}^{(e)}:=g U_{F^{\prime}}^{(e)} g^{-1}$ if $F=g F^{\prime}$ with $g \in G$ and $F^{\prime}$ a facet in $A$. We thus obtain a filtration

$$
P_{F} \supseteq U_{F}^{(0)} \supseteq U_{F}^{(1)} \supseteq \cdots
$$

of the pointwise stabilizer $P_{F}$ by normal subgroups. As in [loc. cit.] we define, for any point $z \in \mathscr{B}$,

$$
U_{z}^{(e)}:=U_{F}^{(e)}
$$

where $F$ is the unique facet of $\mathscr{B}$ that contains $z$. The group $U_{z}^{(e)}$ fixes the point $z$. By construction we have

$$
U_{g z}^{(e)}=g U_{z}^{(e)} g^{-1}
$$

for any $z \in \mathscr{B}$ and any $g \in G$.

Remark. We emphasize that the definition of the groups $\left\{U_{F}^{(e)}\right\}_{F \subset \mathscr{B}, e \geq 0}$ depends on the choice of the special vertex $x_{0}$ as an origin for $A$. We also remark that the very same groups appear in the work of Moy and Prasad on unrefined minimal types [Moy and Prasad 1994; Vignéras 1997].

We will make use of the following basic properties of the groups $U_{F}^{(e)}$. To formulate them let

$$
\Phi=\Phi^{+} \cup \Phi^{-}
$$

be any fixed decomposition of $\Phi$ into positive and negative roots.

Proposition 4.1.7 [Schneider and Stuhler 1997, Propositions I.2.7 and I.2.11 and Corollary I.2.9]. (i) Let $F \subset A$ be a facet. For any $e \geq 0$ the product map induces a bijection

$$
\left(\prod_{\alpha \in \Phi^{-}} U_{f_{F}^{*}+e} \cap U_{\alpha}\right) \times T^{(e)} \times\left(\prod_{\alpha \in \Phi^{+}} U_{f_{F}^{*}+e} \cap U_{\alpha}\right) \stackrel{\sim}{\longrightarrow} U_{F}^{(e)}
$$

whatever ordering of the factors of the left hand side we choose. Moreover, we have

$$
U_{f_{F}^{*}+e} \cap U_{\alpha}=U_{\alpha, f_{F}^{*}(\alpha)+e}
$$

for any $\alpha \in \Phi$.

(ii) For any facet $F \subset \mathscr{B}$ the $U_{F}^{(e)}$ for $e \geq 0$ form a fundamental system of compact open neighborhoods of 1 in $G$,

(iii) $U_{F^{\prime}}^{(e)} \subseteq U_{F}^{(e)}$ for any two facets $F, F^{\prime}$ in $\mathscr{B}$ such that $F^{\prime} \subseteq \bar{F}$. 
4.1.8. As an example and in view of later applications we give a more concrete description of the groups $\left\{U_{x_{0}}^{(e)}\right\}_{e \geq 0}$. The stabilizer $P_{\left\{x_{0}\right\}}$ in $G$ of the vertex $x_{0}$ is a special, good, maximal compact open subgroup of $G$ [Cartier 1979, 3.5]. We let $\mathfrak{G}$ be the connected reductive $o_{L}$-group scheme with generic fiber $\boldsymbol{G}$ associated with the special vertex $x_{0}$ [Tits 1979, 3.4; Bruhat and Tits 1984, 4.6.22]. Its group of $o_{L}$-valued points $\mathfrak{G}\left(o_{L}\right)$ can be identified with $P_{\left\{x_{0}\right\}}$. For $e \geq 0$ we therefore have in $P_{\left\{x_{0}\right\}}$ the normal subgroup $\mathfrak{G}\left(\varpi^{e}\right):=\operatorname{ker}\left(\mathfrak{G}\left(o_{L}\right) \rightarrow \mathfrak{G}\left(o_{L} / \varpi^{e} o_{L}\right)\right)$.

Now the concave function $f_{\left\{x_{0}\right\}}$ vanishes identically whence $f_{\left\{x_{0}\right\}}^{*}$ has constant value $0+$. Thus,

$$
U_{\alpha, f_{\left\{x_{0}\right\}}^{*}(\alpha)+e}=\bigcup_{s>0}\left\{a \in L: v_{L}(a) \geq e+s\right\}=\varpi^{e+1} o_{L}
$$

for any $e \geq 0$. By Proposition 4.1.7(i) and the definition of $T^{(e)}$ we therefore have a canonical isomorphism $U_{x_{0}}^{(e)} \stackrel{\sim}{\longrightarrow} \mathfrak{G}\left(\varpi^{e+1}\right)$ for any $e \geq 0$.

4.2. The Schneider-Stuhler construction. We now review the construction of a certain "localization" functor constructed by P. Schneider and U. Stuhler [1997, IV.1]. In fact, there will be a functor for each "level" $e \geq 0$. Following [loc. cit.], we will suppress this dependence in our notation. In [Schneider and Stuhler 1997] only complex representations are considered. However, all results remain true over our characteristic zero field $K$ [Vignéras 1997].

4.2.1. Recall that a smooth representation $V$ of $G$ is a $K$-vector space $V$ together with a linear action of $G$ such that the stabilizer of each vector is open in $G$. A morphism between two such representations is simply a $K$-linear $G$-equivariant map.

Now let us fix an integer $e \geq 0$ and let $V$ be a smooth representation. For any subgroup $U \subseteq G$ we have the $K$-vector space

$$
V_{U}:=\text { maximal quotient of } V \text { on which the } U \text {-action is trivial }
$$

of $U$-coinvariants of $V$. For any open subset $\Omega \subseteq \mathscr{B}$ we let

$$
\underset{\approx}{V}(\Omega):=K \text {-vector space of all maps } s: \Omega \rightarrow \bigcup_{z \in \Omega} V_{U_{z}^{(e)}} \text { such that }
$$

- $s(z) \in V_{U_{z}^{(e)}}$ for all $z \in \Omega$,

- there is an open covering $\Omega=\bigcup_{i \in I} \Omega_{i}$ and vectors $v_{i} \in V$ with

$$
s(z)=\text { class of } v_{i} \in V_{U_{z}^{(e)}}
$$

for any $z \in \Omega_{i}$ and $i \in I$.

We summarize some properties of this construction in the following proposition. Recall that a sheaf on a polysimplicial space is called constructible if its restriction 
to a given geometric polysimplex is a constant sheaf [Kashiwara and Schapira 1990, 8.1].

Proposition 4.2.2. (i) The correspondence $\Omega \mapsto \underset{\approx}{V}(\Omega)$ is a sheaf of $K$-vector spaces.

(ii) For any $z \in \mathscr{B}$ the stalk of the sheaf $\underset{\approx}{V}$ at $z$ equals $(\underset{\approx}{V})_{z}=V_{U_{z}^{(e)}}$.

(iii) $\underset{\approx}{V}$ is a constructible sheaf whose restriction to any facet $F$ of $\mathscr{B}$ is constant with value $V_{U_{F}^{(e)}}$.

(iv) The correspondence $V \mapsto \underset{\approx}{V}$ is an exact functor from smooth $G$-representations to sheaves of $K$-vector spaces on $\mathscr{B}$.

Proof. Part (i) follows from the local nature of the preceding definition. Part (ii) and (iii) are [Schneider and Stuhler 1997, Lemma IV.1.1]. Part (iv) follows from (ii) because of $\operatorname{char}(K)=0$.

We recall that the smooth representation $V$ is called admissible if the $H$-invariants $V^{H}$ form a finite dimensional $K$-vector space for any compact open subgroup $H$ of $G$. In this situation the natural projection map $V \rightarrow V_{H}$ induces a linear isomorphism $V^{H} \stackrel{\sim}{\longrightarrow} V_{H}$. For an admissible representation $V$ we may therefore deduce from Proposition 4.2.2(ii) that the stalks of $\underset{\approx}{V}$ are finite dimensional $K$-vector spaces.

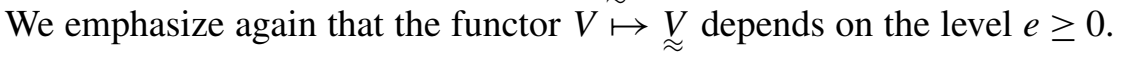

4.3. p-valuations on certain stabilizer subgroups. We keep the notations from the preceding paragraph and define certain $p$-valuations on the groups $U_{F}^{(e)}$. However, for the rest of this section we assume $L=\mathbb{Q}_{p}$.

Lemma 4.3.1. Let $F$ be a facet in $\mathscr{B}$ and $e, e^{\prime} \geq 0$. The commutator group $\left(U_{F}^{(e)}, U_{F}^{\left(e^{\prime}\right)}\right)$ satisfies

$$
\left(U_{F}^{(e)}, U_{F}^{\left(e^{\prime}\right)}\right) \subseteq U_{F}^{\left(e+e^{\prime}\right)} .
$$

Proof. Choosing a facet $F^{\prime}$ in $A$ and an element $g \in G$ such that $F^{\prime}=g F$ we may assume that $F$ lies in $A$. Define a function $h_{F}: \Phi \cup\{0\} \rightarrow \tilde{\mathbb{R}}$ via $\left.h_{F}\right|_{\Phi}:=f_{F}^{*}$ and $h_{F}(0):=0+$. Then $g:=h_{F}+e$ and $f:=h_{F}+e^{\prime}$ are concave functions in the sense of [Bruhat and Tits 1972, Definition 6.4.3]. Consider the function $h: \Phi \cup\{0\} \rightarrow \tilde{\mathbb{R}} \cup\{-\infty\}$ defined as

$$
h(a):=\inf \left\{\sum_{i} f\left(a_{i}\right)+\sum_{j} g\left(b_{j}\right)\right\}
$$

where the infimum is taken over the set of pairs of finite nonempty sets $\left(a_{i}\right)$ and $\left(b_{j}\right)$ of elements in $\Phi \cup\{0\}$ such that $a=\sum_{i} a_{i}+\sum_{j} b_{j}$. Using that the functions $f$ and $g$ are concave one finds

$$
h_{F}(a)+e+e^{\prime} \leq h(a)
$$


for any $a \in \Phi \cup\{0\}$. By [loc. cit., Proposition 6.4.44], the function $h$ is therefore concave and has the property $\left(U_{f}, U_{g}\right) \subseteq U_{h} \subseteq U_{h_{F}+e+e^{\prime}}$. Here, the groups involved are defined completely analogous to (4.1.3) (see [loc. cit., Definition 6.4.42]). It remains to observe that $U_{h_{F}+a}=U_{F}^{(a)}$ for any integer $a \geq 0$ [Schneider and Stuhler 1997, p. 21].

Let $l$ be the rank of the torus $\boldsymbol{T}$. By construction of $\mathfrak{T}$ any trivialization $\boldsymbol{T} \simeq\left(\mathbb{G}_{m}\right)^{l}$ yields an identification $\mathfrak{T} \simeq\left(\mathbb{G}_{m / o_{L}}\right)^{l}$ which makes the structure of the topological groups $T^{(e)}, e \geq 0$ explicit. Moreover, we assume in the following $e \geq 2$. For each $g \in U_{F}^{(e)} \backslash\{1\}$ let

$$
\omega_{F}^{(e)}(g):=\sup \left\{n \geq 0: g \in U_{F}^{(n)}\right\} .
$$

The following corollary is essentially due to H. Frommer [2003, 1.3, proof of Proposition 6]. For sake of completeness we include a proof.

Corollary 4.3.2. The function

$$
\omega_{F}^{(e)}: U_{F}^{(e)} \backslash\{1\} \rightarrow(1 /(p-1), \infty) \subset \mathbb{R}
$$

is a p-valuation on $U_{F}^{(e)}$ for $e \geq 2$.

Proof. The first axiom (i) is obvious and (ii) follows from the lemma. Let $g \in U_{F}^{(e)}$ with $n:=\omega_{F}^{(e)}(g)$. We claim $\omega_{F}^{(e)}\left(g^{p}\right)=n+1$. The root space decomposition (Proposition 4.1.7),

$$
m:\left(\prod_{\alpha \in \Phi^{-}} U_{\alpha, f_{F}^{*}(\alpha)+n}\right) \times T^{(n)} \times\left(\prod_{\alpha \in \Phi^{+}} U_{\alpha, f_{F}^{*}(\alpha)+n}\right) \stackrel{\sim}{\longrightarrow} U_{F}^{(n)},
$$

is in an obvious sense compatible with variation of the level $n$. If $g \in T^{(n)}$ the claim is immediate. The same is true if $g \in U_{\alpha, f_{F}^{*}(\alpha)+n}$ for some $\alpha \in \Phi$ : indeed the filtration of $U_{\alpha}$ is induced by the $p$-adic valuation on $\mathbb{Q}_{p}$ via $x_{\alpha}: \mathbb{Q}_{p} \simeq U_{\alpha}$. In general let $m\left(h_{1}, \ldots, h_{d}\right)=g$. By what we have just said there is $1 \leq i \leq d$ such that $\omega^{(e)}\left(h_{i}^{p}\right)=n+1$ and $\omega^{(e)}\left(h_{j}^{p}\right) \geq n+1$ for all $j \neq i$. Furthermore, $h_{1}^{p} \cdots h_{d}^{p} g^{\prime}=g^{p}$, where $g^{\prime} \in\left(U_{F}^{(n)}, U_{F}^{(n)}\right) \subseteq U_{F}^{(2 n)}$. Since $n \geq 2$ we have $2 n \geq n+2$ and hence $g^{p} \in U_{F}^{(n+1)}$. If $g^{p} \in U_{F}^{(n+2)}$ then $h_{1}^{p} \cdots h_{d}^{p}=g^{p} g^{\prime-1} \in U_{F}^{(n+2)}$, which contradicts the existence of $h_{i}$. Hence $\omega^{(e)}\left(g^{p}\right)=n+1$, which verifies axiom (iii).

4.3.3. For a given root $\alpha \in \Phi$ let $u_{\alpha}$ be a topological generator for the group $U_{\alpha, f_{F}^{*}(\alpha)+e}$. Let $t_{1}, \ldots, t_{l}$ be topological generators for the group $T^{(e)}$. In the light of the decomposition of Proposition 4.1.7(i) it is easy to see that the set

$$
\left\{u_{\alpha}\right\}_{\alpha \in \Phi^{-}} \cup\left\{t_{i}\right\}_{i=1, \ldots, l} \cup\left\{u_{\alpha}\right\}_{\alpha \in \Phi^{+}}
$$

arranged in the order suggested in Frommer's proof is an ordered basis for the $p$-valued group $\left(U_{F}^{(e)}, \omega_{F}^{(e)}\right)$. Of course, $\omega_{F}^{(e)}(h)=e$ for any element $h$ of this ordered basis. 
For technical reasons we will work in the following with the slightly simpler $p$-valuations

$$
\stackrel{\circ}{\omega}_{F}^{(e)}:=\omega_{F}^{(e)}-(e-1)
$$

satisfying $\stackrel{\circ}{\omega}_{F}^{(e)}(h)=1$ for any element $h$ of the above ordered basis. If $z \in \mathscr{B}$ lies in the facet $F \subset \mathscr{B}$ we write $\stackrel{\circ}{\omega}_{z}^{(e)}$ for $\stackrel{(}{\omega}_{F}^{(e)}$.

Remark 4.3.4. The tangent map at $1 \in H:=U_{F}^{(2)}$ corresponding to the $p$-power map equals multiplication by $p$ and thus, is an isomorphism. It follows from Proposition 4.1.7(ii) that there is $e(F) \geq 2$ such that for any $e \geq e(F)$ any element $g \in U_{F}^{(e+1)}$ is a $p$-th power $h^{p}$ with $h \in H$. Since $H$ is $p$-valued, axiom (iii) implies $h \in U_{F}^{(e)}$. This means that $\left(U_{F}^{(e)}, \stackrel{\leftrightarrow}{\omega}_{F}^{(e)}\right)$ is $p$-saturated. For $e \geq e(F)$ the group $U_{F}^{(e)}$ is therefore a uniform pro- $p$ group (apply remark before Lemma 4.4 in [Schneider and Teitelbaum 2003] to $\stackrel{\leftrightarrow}{\omega}_{F}^{(e)}$ and use $p \neq 2$ ). Since any facet in $\mathscr{B}$ is conjugate to a facet in $\mathscr{C}$ we deduce from (4.1.6) that there is a number $e_{\text {uni }} \geq 2$ such that all the groups $U_{F}^{(e)}$ for $F \subset \mathscr{B}$ are uniform pro- $p$ groups whenever $e \geq e_{\text {uni. }}$ In this situation, Proposition A1 of [Huber et al. 2011] asserts that the subgroups

$$
U_{F}^{(e)} \supset U_{F}^{(e+1)} \supset U_{F}^{(e+2)} \supset \cdots
$$

form the lower $p$-series of the group $U_{F}^{(e)}$.

For technical reasons that will become apparent in Section 7.4 we include the following additional property into the definition of $e_{\text {uni. }}$. Let $\overline{\mathscr{C}}$ be the closure of the fundamental chamber $\mathscr{C} \subset A$ and let $x_{0} \in A$ be the chosen origin; see Section 4.1.2. We choose once and for all $e_{\text {uni }} \geq 2$ such that, for $e \geq e_{\text {uni }}$, all groups $U_{F}^{(e)}$ are uniform pro- $p$ groups and such that $U_{z}^{(e)} \subseteq U_{w x_{0}}^{(0)}$ for all points $z \in \overline{\mathscr{C}}$ and all $w \in W$.

We may apply the discussion of Section 2.1 to $\left(U_{F}^{(e)}, \stackrel{(}{\omega}_{F}^{(e)}\right)$ and the above ordered basis to obtain a family of norms $\|\cdot\|_{r}, r \in[1 / p, 1)$ on $D\left(U_{F}^{(e)}, K\right)$ with completions $D_{r}\left(U_{F}^{(e)}, K\right)$ being $K$-Banach algebras. For facets $F, F^{\prime}$ in $\mathscr{B}$ such that $F^{\prime} \subseteq \bar{F}$ we shall need a certain "gluing" lemma for these algebras.

Lemma 4.3.5. Let $F, F^{\prime}$ be two facets in $\mathscr{B}$ such that $F^{\prime} \subseteq \bar{F}$. The inclusion $U_{F^{\prime}}^{(e)} \subseteq U_{F}^{(e)}$ extends to a norm-decreasing algebra homomorphism

$$
\sigma_{r}^{F^{\prime} F}: D_{r}\left(U_{F^{\prime}}^{(e)}, K\right) \rightarrow D_{r}\left(U_{F}^{(e)}, K\right) .
$$

Moreover, (i) $\sigma_{r}^{F F}=\mathrm{id}$ and (ii) $\sigma_{r}^{F^{\prime} F} \circ \sigma_{r}^{F^{\prime \prime} F^{\prime}}=\sigma_{r}^{F^{\prime \prime} F}$ if $F^{\prime \prime}$ is a third facet in $\mathscr{B}$ with $F^{\prime \prime} \subseteq \overline{F^{\prime}}$.

Finally, $\sigma_{r}^{F^{\prime} F}$ restricted to $\operatorname{Lie}\left(U_{F^{\prime}}^{(e)}\right)$ equals the map $\operatorname{Lie}\left(U_{F^{\prime}}^{(e)}\right) \simeq \operatorname{Lie}\left(U_{F}^{(e)}\right) \subset$ $D_{r}\left(U_{F}^{(e)}, K\right)$ where the first arrow is the canonical Lie algebra isomorphism from [Bourbaki 1972, III Section 3.8]. 
Proof. By functoriality [Kohlhaase $2007,1.1$ ] of $D(\cdot, K)$ we obtain an algebra homomorphism

$$
\sigma: D\left(U_{F^{\prime}}^{(e)}, K\right) \rightarrow D\left(U_{F}^{(e)}, K\right) .
$$

Let $h_{1}^{\prime}, \ldots, h_{d}^{\prime}$ and $h_{1}, \ldots, h_{d}$ be the ordered bases of $U_{F^{\prime}}^{(e)}$ and $U_{F}^{(e)}$ respectively. Let $b_{i}^{\prime}=h_{i}^{\prime}-1 \in \mathbb{Z}\left[U_{F^{\prime}}^{(e)}\right]$ and $\boldsymbol{b}^{\prime m}:=b_{1}^{\prime m_{1}} \cdots b_{d}^{\prime m_{d}}$ for $m \in \mathbb{N}_{0}^{d}$. Given an element

$$
\lambda=\sum_{m \in \mathbb{N}_{0}^{d}} d_{m} \boldsymbol{b}^{\prime m} \in D\left(U_{F^{\prime}}^{(e)}, K\right)
$$

we have $\|\lambda\|_{r}=\sup _{m}\left|d_{m}\right|\left\|b_{i}^{\prime}\right\|_{r}$. Because

$$
\left.\|\sigma(\lambda)\|_{r} \leq \sup _{m}\left|d_{m}\right|\left(\left\|\sigma\left(b_{1}^{\prime}\right)\right\|_{r}\right)^{m_{1}} \cdots\left(\left\|\sigma\left(b_{d}^{\prime}\right)\right\|_{r}\right)^{m_{d}}\right)
$$

it therefore suffices to prove $\left\|\sigma\left(b_{i}^{\prime}\right)\right\|_{r} \leq\left\|b_{i}^{\prime}\right\|_{r}$ for any $i$. If $h_{i}^{\prime}$ belongs to the toral part of the ordered basis of $U_{F^{\prime}}^{(e)}$ then we may assume $\sigma\left(b_{i}^{\prime}\right)=b_{i}^{\prime}$ and we are done. Let therefore $\alpha \in \Phi$ and consider the corresponding elements $h_{\alpha}^{\prime}$ and $h_{\alpha}$ in the ordered bases of $U_{F^{\prime}}^{(e)}$ and $U_{F}^{(e)}$ respectively. By the root space decomposition we have

$$
U_{\alpha, f_{F^{\prime}}^{*}(\alpha)+e} \subseteq U_{\alpha, f_{F}^{*}(\alpha)+e}=\left(h_{\alpha}\right)^{\mathbb{Z}_{p}} .
$$

Let therefore $a \in \mathbb{Z}_{p}$ such that $h_{\alpha}^{\prime}=\left(h_{\alpha}\right)^{a}$. Since a change of ordered basis does not affect the norms in question (see Section 2.2.3) we may assume $a=p^{s}$ for some natural number $s \geq 0$. Then

$$
h_{\alpha}^{\prime}-1=\left(h_{\alpha}+1-1\right)^{p^{s}}-1=\sum_{k=1, \ldots, p^{s}}\left(\begin{array}{c}
p^{s} \\
k
\end{array}\right)\left(h_{\alpha}-1\right)^{k}
$$

and therefore

$\left\|\sigma\left(h_{\alpha}^{\prime}-1\right)\right\|_{r} \leq \max _{k=1, \ldots, p^{s}}\left|\left(\begin{array}{c}p^{s} \\ k\end{array}\right)\right|\left\|\left(h_{\alpha}-1\right)\right\|_{r}^{k}=\max _{k=1, \ldots, p^{s}}\left|\left(\begin{array}{c}p^{s} \\ k\end{array}\right)\right| r^{k} \leq r=\left\|h_{\alpha}^{\prime}-1\right\|_{r}$

which shows the claim and the existence of $\sigma_{r}^{F F^{\prime}}$. Properties (i) and (ii) in the second paragraph of the statement follow from functoriality of $D(\cdot, K)$ by passing to completions. Since $U_{F^{\prime}}^{(e)} \subseteq U_{F}^{(e)}$ is an open immersion of Lie groups the final statement is clear.

\section{Sheaves on the flag variety and Lie algebra representations}

\subsection{Differential operators on the flag variety.}

5.1.1. Let $X$ denote the variety of Borel subgroups of $\boldsymbol{G}$. It is a smooth and projective $L$-variety. Let $\mathcal{O}_{X}$ be its structure sheaf. Let $\mathfrak{g}$ be the Lie algebra of $\boldsymbol{G}$. 
Differentiating the natural (left) action of $\boldsymbol{G}$ on $X$ yields a homomorphism of Lie algebras

$$
\alpha: \mathfrak{g} \rightarrow \Gamma\left(X, \mathcal{T}_{X}\right)
$$

into the global sections of the tangent sheaf $\mathcal{T}_{X}=\operatorname{Der}_{L}\left(\mathcal{O}_{X}\right)$ of $X$ [Demazure and Gabriel 1970, II Section 4.4.4]. In the following we identify an abelian group (algebra, module etc.) with the corresponding constant sheaf on $X$. This should not cause any confusion. Letting

$$
\mathfrak{g}^{\circ}:=\mathcal{O}_{X} \otimes_{L} \mathfrak{g}
$$

the map $\alpha$ extends to a morphism of $\mathcal{O}_{X}$-modules $\alpha^{\circ}: \mathfrak{g}^{\circ} \rightarrow \mathcal{T}_{X}$. Defining $[\mathfrak{x}, f]:=$ $\alpha(\mathfrak{x})(f)$ for $\mathfrak{x} \in \mathfrak{g}$ and a local section $f$ of $\mathcal{O}_{X}$ makes $\mathfrak{g}^{\circ}$ a sheaf of $L$-Lie algebras. ${ }^{5}$ Then $\alpha^{\circ}$ is a morphism of $L$-Lie algebras. We let $\mathfrak{b}^{\circ}:=\operatorname{ker} \alpha^{\circ}$, a subalgebra of $\mathfrak{g}^{\circ}$, and $\mathfrak{n}^{\circ}:=\left[\mathfrak{b}^{\circ}, \mathfrak{b}^{\circ}\right]$ its derived algebra. At a point $x \in X$ with residue field $\kappa(x)$ the reduced stalks of the sheaves $\mathfrak{b}^{\circ}$ and $\mathfrak{n}^{\circ}$ equal the Borel subalgebra $\mathfrak{b}_{x}$ of $\kappa(x) \otimes_{L} \mathfrak{g}$ defined by $x$ and its nilpotent radical $\mathfrak{n}_{x} \subset \mathfrak{b}_{x}$ respectively. Let $\mathfrak{h}$ denote the abstract Cartan algebra of $\mathfrak{g}$ [Miličić 1993a, Section 2]. We view the $\mathcal{O}_{X}$-module $\mathcal{O}_{X} \otimes_{L} \mathfrak{h}$ as an abelian $L$-Lie algebra. By definition of $\mathfrak{h}$ there is a canonical isomorphism of $\mathcal{O}_{X}$-modules and $L$-Lie algebras

$$
\mathfrak{b}^{\circ} / \mathfrak{n}^{\circ} \stackrel{\sim}{\longrightarrow} \mathcal{O}_{X} \otimes_{L} \mathfrak{h}
$$

Let $U(\mathfrak{g})$ be the enveloping algebra of $\mathfrak{g}$. The enveloping algebra of the Lie algebra $\mathfrak{g}^{\circ}$ has the underlying $\mathcal{O}_{X}$-module $\mathcal{O}_{X} \otimes_{L} U(\mathfrak{g})$. Its $L$-algebra of local sections over an open affine $V \subseteq X$ is the skew enveloping algebra $\mathcal{O}_{X}(V) \# U(\mathfrak{g})$ relative to $\alpha: \mathfrak{g} \rightarrow \operatorname{Der}_{L}\left(\mathcal{O}_{X}(V)\right.$ ) (in the sense of sec 3 ). To emphasize this skew multiplication we follow [Bezrukavnikov et al. 2008, 3.1.3] and denote the enveloping algebra of $\mathfrak{g}^{\circ}$ by

$$
\mathcal{O}_{X} \# U(\mathfrak{g}) .
$$

5.1.3. To bring in the torus $\boldsymbol{T}$ we choose a Borel subgroup $\boldsymbol{B} \subset \boldsymbol{G}$ defined over $L$ containing $\boldsymbol{T}$. Let $\boldsymbol{N} \subset \boldsymbol{B}$ be the unipotent radical of $\boldsymbol{B}$ and let $\boldsymbol{N}^{-}$be the unipotent radical of the Borel subgroup opposite to $\boldsymbol{B}$. We denote by

$$
q: \boldsymbol{G} \rightarrow \boldsymbol{G} / \boldsymbol{B}=X
$$

the canonical projection. Let $\mathfrak{b}$ be the Lie algebra of $\boldsymbol{B}$ and $\mathfrak{n} \subset \mathfrak{b}$ its nilpotent radical. If $\mathfrak{t}$ denotes the Lie algebra of $\boldsymbol{T}$ the map $\mathfrak{t} \subset \mathfrak{b} \rightarrow \mathfrak{b} / \mathfrak{n} \simeq \mathfrak{h}$ induces an isomorphism $\mathfrak{t} \simeq \mathfrak{h}$ of $L$-Lie algebras. We will once and for all identify these two

${ }^{5}$ Following [Beilinson and Bernstein 1981] we call such a sheaf simply a Lie algebra over $X$ in the sequel. This abuse of language should not cause confusion. 
Lie algebras via this isomorphism. Consequently, (5.1.2) yields a morphism of $\mathcal{O}_{X}$-modules and $L$-Lie algebras

$$
\mathfrak{b}^{\circ} \rightarrow \mathfrak{b}^{\circ} / \mathfrak{n}^{\circ} \stackrel{\sim}{\longrightarrow} \mathcal{O}_{X} \otimes_{L} \mathfrak{t} .
$$

Given a linear form $\lambda \in \mathfrak{t}^{*}$ it extends $\mathcal{O}_{X}$-linearly to the target of this morphism and may then be pulled-back to $\mathfrak{b}^{\circ}$. This gives a $\mathcal{O}_{X}$-linear morphism $\lambda^{\circ}: \mathfrak{b}^{\circ} \rightarrow \mathcal{O}_{X}$.

5.1.4. Let $\rho:=\frac{1}{2} \sum_{\alpha \in \Phi^{+}} \alpha$. Given $\chi \in \mathfrak{t}^{*}$ we put $\lambda:=\chi-\rho$. Denote by $\mathcal{I}_{\chi}$ the right ideal sheaf of $\mathcal{O}_{X} \# U(\mathfrak{g})$ generated by $\operatorname{ker} \lambda^{\circ}$, i.e., by the expressions

$$
\xi-\lambda^{\circ}(\xi)
$$

with $\xi$ a local section of $\mathfrak{b}^{\circ} \subset \mathfrak{g}^{\circ} \subset \mathcal{O}_{X} \# U(\mathfrak{g})$. It is a two-sided ideal and we let

$$
\mathcal{D}_{\chi}:=\left(\mathcal{O}_{X} \# U(\mathfrak{g})\right) / \mathcal{I}_{\chi}
$$

be the quotient sheaf. This is a sheaf of noncommutative $L$-algebras on $X$ endowed with a natural algebra morphism $U(\mathfrak{g}) \rightarrow \Gamma\left(X, \mathcal{D}_{\chi}\right)$ induced by $\mathfrak{x} \mapsto 1 \otimes \mathfrak{x}$ for $\mathfrak{x} \in U(\mathfrak{g})$. On the other hand $\mathcal{D}_{\chi}$ is an $\mathcal{O}_{X}$-module through the (injective) $L$-algebra morphism $\mathcal{O}_{X} \rightarrow \mathcal{D}_{\chi}$ induced by $f \mapsto f \otimes 1$. This allows to define the full subcategory $\mathcal{M}_{q c}\left(\mathcal{D}_{\chi}\right)$ of the (left) $\mathcal{D}_{\chi}$-modules consisting of modules which are quasicoherent as $\mathcal{O}_{X}$-modules. It is abelian.

5.1.5. For future reference we briefly discuss a refinement of the above construction of the sheaf $\mathcal{D}_{\chi}$. The right ideal of $\mathcal{O}_{X} \# U(\mathfrak{g})$ generated by $\mathfrak{n}^{\circ}$ is a two-sided ideal and, following [Miličić 1993a, Section 3] we let

$$
\mathcal{D}_{\mathfrak{t}}:=\left(\mathcal{O}_{X} \# U(\mathfrak{g})\right) / \mathfrak{n}^{\circ}\left(\mathcal{O}_{X} \# U(\mathfrak{g})\right)
$$

be the quotient sheaf. We have the open subscheme $U_{1}:=q\left(N^{-}\right)$of $X$. Choose a representative $\dot{w} \in G$ for every $w \in W$ with $\dot{1}=1$. The translates $U_{w}:=\dot{w} U_{1}$ for all $w \in W$ form a Zariski covering of $X$. Let $\mathfrak{n}^{-}$be the Lie algebra of $N^{-}$and put $\mathfrak{n}^{-, w}:=\operatorname{Ad}(\dot{w})\left(\mathfrak{n}^{-}\right)$for any $w \in W$.

For any $w \in W$ there are obvious canonical maps from $\mathcal{O}_{X}\left(U_{w}\right), U\left(\mathfrak{n}^{-, w}\right)$ and $U(\mathfrak{t})$ to $\mathcal{O}_{X}\left(U_{w}\right) \# U(\mathfrak{g})$ and therefore to $\mathcal{D}_{\mathfrak{t}}\left(U_{w}\right)$. According to [Miličić 1993b, Lemma C.1.3] they induce a $K$-algebra isomorphism

$$
\left(\mathcal{O}_{X}\left(U_{w}\right) \# U\left(\mathfrak{n}^{-, w}\right)\right) \otimes_{L} U(\mathfrak{t}) \stackrel{\sim}{\longrightarrow} \mathcal{D}_{\mathfrak{t}}\left(U_{w}\right) .
$$

Note that $N^{-} \cong \mathbb{A}_{L}^{\left|\Phi^{-}\right|}$implies that the skew enveloping algebra $\mathcal{O}_{X}\left(U_{w}\right) \# U\left(\mathfrak{n}^{-, w}\right)$ is equal to the usual algebra of differential operators $\mathcal{D}_{X}\left(U_{w}\right)$ on the translated affine space $U_{w}=\dot{w} U_{1}$.

The above discussion implies that the canonical homomorphism

$$
U(\mathfrak{t}) \rightarrow \mathcal{O}_{X} \# U(\mathfrak{g}), \mathfrak{x} \mapsto 1 \otimes \mathfrak{x}
$$


induces a central embedding $U(\mathfrak{t}) \hookrightarrow \mathcal{D}_{\mathfrak{t}}$. In particular, the sheaf $(\operatorname{ker} \lambda) \mathcal{D}_{\mathfrak{t}}$ is a two-sided ideal in $\mathcal{D}_{\mathfrak{t}}$. According to the discussion before Theorem 3.2 in [Miličić 1993a, p. 138], the canonical map $\mathcal{D}_{\mathfrak{t}} \rightarrow \mathcal{D}_{\chi}$ coming from $\mathfrak{n}^{\circ} \subset \operatorname{ker} \lambda^{\circ}$ induces

$$
\mathcal{D}_{\mathfrak{t}} \otimes_{U(\mathfrak{t})} L_{\lambda}=\mathcal{D}_{\mathfrak{t}} /(\operatorname{ker} \lambda) \mathcal{D}_{\mathfrak{t}} \stackrel{\sim}{\rightarrow} \mathcal{D}_{\chi},
$$

an isomorphism of sheaves of $K$-algebras.

Remark. According to the above we may view the formation of the sheaf $\mathcal{D}_{\chi}$ as a two-step process. In a first step on constructs the sheaf $\mathcal{D}_{\mathfrak{t}}$ whose sections over the Weyl translates of the big cell $U_{1}$ are explicitly computable. Secondly, one performs a central reduction $\mathcal{D}_{\mathfrak{t}} \otimes_{U(\mathfrak{t})} L_{\lambda}$ via the chosen character $\lambda=\chi-\rho$. This point of view will be useful in later investigations.

\subsection{The Beilinson-Bernstein localization theorem.}

5.2.1. We recall some notions related to the classical Harish-Chandra isomorphism. To begin with let $S(\mathfrak{t})$ be the symmetric algebra of $\mathfrak{t}$ and let $S(\mathfrak{t})^{W}$ be the subalgebra of Weyl invariants. Let $Z(\mathfrak{g})$ be the center of the universal enveloping algebra $U(\mathfrak{g})$ of $\mathfrak{g}$. The classical Harish-Chandra map is an algebra isomorphism $Z(\mathfrak{g}) \stackrel{\sim}{\longrightarrow} S(\mathfrak{t})^{W}$ relating central characters and highest weights of irreducible highest weight $\mathfrak{g}$ modules in a meaningful way [Dixmier 1996, 7.4]. Given a linear form $\chi \in \mathfrak{t}^{*}$ we let

$$
\sigma(\chi): Z(\mathfrak{g}) \rightarrow L
$$

denote the central character associated with $\chi$ via the Harish-Chandra map. Recall that $\chi \in \mathfrak{t}^{*}$ is called dominant if $\chi(\check{\alpha}) \notin\{-1,-2, \ldots\}$ for any coroot $\check{\alpha}$ with $\alpha \in \Phi^{+}$. It is called regular if $w(\chi) \neq \chi$ for any $w \in W$ with $w \neq 1$.

Let $\theta:=\sigma(\chi)$ and put $U(\mathfrak{g})_{\theta}:=U(\mathfrak{g}) \otimes_{Z(\mathfrak{g}), \theta} L$ for the corresponding central reduction.

Theorem 5.2.2 [Beilinson and Bernstein 1981].

(i) The algebra morphism $U(\mathfrak{g}) \rightarrow \Gamma\left(X, \mathcal{D}_{\chi}\right)$ induces an isomorphism $U(\mathfrak{g})_{\theta} \simeq$ $\Gamma\left(X, \mathcal{D}_{\chi}\right)$.

(ii) If $\chi$ is dominant and regular the functor $M \mapsto \mathcal{D}_{\chi} \otimes_{U(\mathfrak{g})_{\theta}} M$ is an equivalence of categories between the (left) $U(\mathfrak{g})_{\theta}$-modules and $\mathcal{M}_{q c}\left(\mathcal{D}_{\chi}\right)$.

(iii) Let $M$ be a $U(\mathfrak{g})_{\theta}$-module. The reduced stalk of the sheaf $\mathcal{D}_{\chi} \otimes_{U(\mathfrak{g})_{\theta}} M$ at a point $x \in X$ equals the $\lambda$-coinvariants of the $\mathfrak{h}$-module $\left(\kappa(x) \otimes_{L} M\right) / \mathfrak{n}_{x}\left(\kappa(x) \otimes_{L}\right.$ $M)$.

Remarks. (i) In [Beilinson and Bernstein 1981] the theorem is proved under the assumption that the base field is algebraically closed. However, all proofs in that paper go through over an arbitrary characteristic zero field in the case 
where the Lie algebra $\mathfrak{g}$ is split over the base field. In the following, this is the only case we shall require.

(ii) If $\lambda:=\chi-\rho \in X^{*}(\boldsymbol{T}) \subset \mathfrak{t}^{*}$ and if $\mathcal{O}(\lambda)$ denotes the associated invertible sheaf on $X$ then $\mathcal{D}_{\chi}$ can be identified with the sheaf of differential endomorphisms of $\mathcal{O}(\lambda)$ [Miličic 1993a, p. 138]. It is therefore a twisted sheaf of differential operators on $X$ in the sense of [Beilinson and Bernstein 1981, Section 1]. In particular, if $\chi=\rho$ the map $\alpha^{\circ}$ induces an isomorphism $\mathcal{D}_{\rho} \stackrel{\sim}{\longrightarrow} \mathcal{D}_{X}$ with the usual sheaf of differential operators on $X$ [Grothendieck 1967, Section 16.8]. In this case, $\mathcal{M}_{q c}\left(\mathcal{D}_{\chi}\right)$ equals therefore the usual category of algebraic $D$ modules on $X$ in the sense of [Borel et al. 1987].

\section{Berkovich analytifications}

\subsection{Differential operators on the analytic flag variety.}

6.1.1. For the theory of Berkovich analytic spaces we refer to [Berkovich 1990; 1993]. We keep the notations introduced in the preceding section. In particular, $X$ denotes the variety of Borel subgroups of $\boldsymbol{G}$. Being a scheme of finite type over $L$ we have an associated Berkovich analytic space $X^{\text {an }}$ over $L$ [loc. cit., Theorem 3.4.1]. In the preceding section we recalled a part of the algebraic Beilinson-Bernstein localization theory over $X$. It admits the following "analytification" over $X^{\text {an }}$.

By construction $X^{\text {an }}$ comes equipped with a canonical morphism

$$
\pi: X^{\text {an }} \rightarrow X
$$

of locally ringed spaces. Let $\pi^{*}$ be the associated inverse image functor from $\mathcal{O}_{X}$-modules to $\mathcal{O}_{X}$ an-modules. Here $\mathcal{O}_{X}$ an denotes the structure sheaf of the locally ringed space $X^{\text {an }}$. As with any morphism of locally ringed spaces we have the sheaf

$$
\mathcal{T}_{X} \text { an }:=\operatorname{Der}_{L}\left(\mathcal{O}_{X} \text { an }\right)
$$

of $L$-derivations of $\mathcal{O}_{X}$ an [Grothendieck 1967, 16.5.4]. By definition $\Gamma\left(X^{\text {an }}, \mathcal{T}_{X}\right.$ an $)=$ $\operatorname{Der}_{L}\left(\mathcal{O}_{X}\right.$ an $)$. Since $X^{\text {an }}$ is smooth over $L$ the results of [Berkovich 1993, 3.3 and 3.5] imply that the stalk of this sheaf at a point $x \in X^{\text {an }}$ equals $\mathcal{T}_{X}{ }^{\text {an }}, x=\operatorname{Der}_{L}\left(\mathcal{O}_{X^{\text {an }}, x}\right)$.

Let $\boldsymbol{G}^{\text {an }}$ denote the analytic space associated to the variety $\boldsymbol{G}$ and let $\pi_{\boldsymbol{G}}: \boldsymbol{G}^{\text {an }} \rightarrow \boldsymbol{G}$ be the canonical morphism. The space $G^{\text {an }}$ is a group object in the category of $L$-analytic spaces (a L-analytic group in the terminology of [Berkovich 1990, 5.1]). The unit sections of $\boldsymbol{G}$ and $\boldsymbol{G}^{\text {an }}$ correspond via $\pi_{\boldsymbol{G}}$, which allows us to canonically identify the Lie algebra of $\boldsymbol{G}^{\text {an }}$ with $\mathfrak{g}$ [loc. cit., Theorem 3.4.1(ii)]. By functoriality the group $G^{\text {an }}$ acts on $X^{\text {an }}$. The following result is proved as in the scheme case.

Lemma 6.1.2. The group action induces a Lie algebra homomorphism

$$
\mathfrak{g} \rightarrow \Gamma\left(X^{\mathrm{an}}, \mathcal{T}_{X^{\mathrm{an}}}\right) .
$$


We define

$$
\mathfrak{g}^{\circ, \text { an }}:=\mathcal{O}_{X} \text { an } \otimes_{L} \mathfrak{g}=\pi^{*}\left(\mathfrak{g}^{\circ}\right) .
$$

The preceding lemma allows on the one hand, to define a structure of $L$-Lie algebra on $\mathfrak{g}^{\circ \text {,an }}$. The respective enveloping algebra will be denoted by $\mathcal{O}_{X}$ an $\# U(\mathfrak{g})$. On the other hand, the map from the lemma extends to a $\mathcal{O}_{X}$ an-linear morphism of $L$-Lie algebras

$$
\alpha^{\circ, \text { an }}: \mathfrak{g}^{\circ, a n} \rightarrow \mathcal{T}_{X} \text { an }
$$

As in the algebraic case we put $\mathfrak{b}^{\circ, \text { an }}:=\operatorname{ker} \alpha^{\circ \text {,an }}$ and $\mathfrak{n}^{\circ \text {,an }}:=\left[\mathfrak{b}^{\circ \text {,an }}, \mathfrak{b}^{\circ, \text { an }}\right]$. Again, we obtain a morphism $\mathfrak{b}^{\circ \text {,an }} \rightarrow \mathcal{O}_{X}$ an $\otimes_{L} \mathfrak{t}$. Given $\chi \in \mathfrak{t}^{*}$ and $\lambda:=\chi-\rho$ we denote by

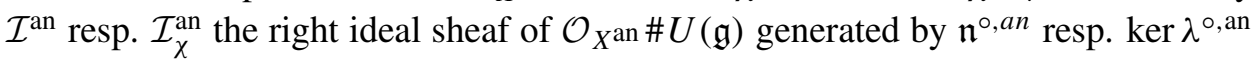
where $\lambda^{\circ \text {,an }}$ equals the $\mathcal{O}_{X}$ an-linear form of $\mathfrak{b}^{\circ \text {,an }}$ induced by $\lambda$. These are two-sided ideals. We let

$$
\mathcal{D}_{\mathfrak{t}}^{\text {an }}:=\left(\mathcal{O}_{X} \text { an } \# U(\mathfrak{g})\right) / \mathcal{I}^{\text {an }} \quad \text { and } \quad \mathcal{D}_{\chi}^{\text {an }}:=\left(\mathcal{O}_{X} \text { an } \# U(\mathfrak{g})\right) / \mathcal{I}_{\chi}^{\text {an }}
$$

be the quotient sheaves. We view $\mathcal{D}_{\chi}^{\text {an }}$ as a sheaf of twisted differential operators on $X^{\text {an }}$.

All these constructions are compatible with their algebraic counterparts via the functor $\pi^{*}$. For example, using the fact that $\pi^{*}\left(\mathcal{T}_{X}\right)=\mathcal{T}_{X}$ an it follows from the above proof that $\alpha^{\circ \text {,an }}=\pi^{*}\left(\alpha^{\circ}\right)$. Moreover, all that has been said in Section 5 on the relation between the sheaves $\mathcal{D}_{\mathrm{t}}$ and $\mathcal{D}_{\chi}$ remains true for its analytifications. In particular, $\mathcal{D}_{\chi}^{\text {an }}$ is a central reduction of $\mathcal{D}_{\mathfrak{t}}^{\text {an }}$ via the character $\lambda: U(\mathfrak{t}) \rightarrow L$ :

$$
\mathcal{D}_{\mathfrak{t}}^{\text {an }} /(\operatorname{ker} \lambda) \mathcal{D}_{\mathfrak{t}}^{\text {an }} \stackrel{\sim}{\longrightarrow} \mathcal{D}_{\chi}^{\text {an }} .
$$

6.2. The Berkovich embedding and analytic stalks. Recall our chosen Borel subgroup $\boldsymbol{B} \subset \boldsymbol{G}$ containing $\boldsymbol{T}$ and the quotient morphism $q: \boldsymbol{G} \rightarrow \boldsymbol{G} / \boldsymbol{B}=X$. We will make heavy use of the following result of V. Berkovich which was taken up and generalized in a conceptual way by B. Rémy, A. Thuillier and A. Werner. Let $\eta \in X$ be the generic point of $X$.

Theorem 6.2.1. There exists a G-equivariant injective map

$$
\vartheta_{\boldsymbol{B}}: \mathscr{B} \rightarrow X^{\text {an }}
$$

which is a homeomorphism onto its image. The latter is a locally closed subspace of $X^{\text {an }}$ contained in the preimage $\pi^{-1}(\eta)$ of the generic point of $X$.

Proof. This is [Berkovich 1990, 5.5.1]. We sketch the construction in the language of [Rémy et al. 2010]. The map is constructed in three steps. First one attaches to any point $z \in \mathscr{B}$ an $L$-affinoid subgroup $\boldsymbol{G}_{z}$ of $\boldsymbol{G}^{\text {an }}$ whose rational points coincide with the stabilizer of $z$ in $G$. In a second step one attaches to $\boldsymbol{G}_{z}$ the unique point in its Shilov boundary (the sup-norm on $\boldsymbol{G}_{z}$ ) which defines a map $\vartheta: \mathscr{B} \rightarrow \boldsymbol{G}^{\text {an }}$. 
In a final step one composes this map with the analytification of the orbit map $\boldsymbol{G} \rightarrow X, g \mapsto g . \boldsymbol{B}$. The last assertion follows from the next lemma.

Lemma 6.2.2. Let $z \in \mathscr{B}$. The local rings $\mathcal{O}_{X^{\mathrm{an}}, \vartheta_{\boldsymbol{B}}(z)}$ and $\mathcal{O}_{X, \pi\left(\vartheta_{\boldsymbol{B}}(z)\right)}$ are fields. In particular, $\left.\pi\left(\vartheta_{\boldsymbol{B}}(z)\right)\right)=\eta$, the generic point of $X$.

Proof. This is a direct consequence of [Rémy et al. 2010, Corollary 2.18] and the sentence immediately following that corollary.

Since $X^{\text {an }}$ is a compact Hausdorff space by [Berkovich 1990, 3.4.8], the closure of the image of $\vartheta_{\boldsymbol{B}}$ in $X^{\text {an }}$ is a compactification of $\mathscr{B}$ [loc. cit., Remark 3.31]. It is called the Berkovich compactification of $\mathscr{B}$ of type $\varnothing$ [loc. cit., Definition 3.30]. We will in the following often identify $\mathscr{B}$ with its image under $\vartheta_{\boldsymbol{B}}$ and hence, view $\mathscr{B}$ as a locally closed subspace of $X^{\text {an }}$.

6.2.3. By [Berkovich 1993, 1.5] the space $X^{\text {an }}$ is a good analytic space (in the sense of [loc. cit., Remark 1.2.16] which means that any point of $X^{\text {an }}$ lies in the topological interior of an affinoid domain. In particular, given $x \in X^{\text {an }}$ the stalk $\mathcal{O}_{X^{a n}, x}$ may be written as

$$
\mathcal{O}_{X}{ }^{\mathrm{an}}, x=\underset{x \in V}{\lim } \mathcal{A}_{V}
$$

where the inductive limit ranges over the affinoid neighborhoods $V$ of $x$ and where $\mathcal{A}_{V}$ denotes the associated affinoid algebra. As usual a subset of neighborhoods of $x$ will be called cofinal if it is cofinal in the directed partially ordered set of all neighborhoods of $x$. If $V$ is an affinoid neighborhood of $x$, the corresponding affinoid algebra $\mathcal{A}_{V}$ carries its Banach topology. We endow $\mathcal{O}_{X}{ }^{\text {an }}, x$ with the locally convex final topology [Schneider 2002, Section 5.E] arising from the above inductive limit. This topology makes $\mathcal{O}_{X}{ }^{\text {an }}{ }_{, x}$ a topological $L$-algebra. We need another, rather technical, property of this topology.

Lemma 6.2.4. Let $x \in X^{\text {an }}$. There is a sequence $V_{1} \supset V_{2} \supset V_{3} \supset$... of irreducible reduced strictly affinoid neighborhoods of $x$ which is cofinal and has the property: the homomorphism of affinoid algebras $\mathcal{A}_{V_{i}} \rightarrow \mathcal{A}_{V_{i+1}}$ associated with the inclusion $V_{i+1} \subset V_{i}$ is flat and an injective compact linear map between Banach spaces. In particular, the stalk $\mathcal{O}_{X^{\mathrm{an}}}{ }_{, x}$ is a vector space of compact type.

Proof. Being an analytification of a variety over $L$, the analytic space $X^{\text {an }}$ is closed (in the sense of [Berkovich 1990, p. 49]); cf. [loc. cit., 3.4.1]. Since the valuation on $L$ is nontrivial, $X^{\text {an }}$ is strictly $k$-analytic [loc. cit., Proposition 3.1.2]. Let $V$ be a strictly affinoid neighborhood of $x$ in $X^{\text {an }}$ so that $x$ lies in the topological interior of $V$. In the following we will use basic results on the relative interior $\operatorname{Int}(Y / Z)$ of an analytic morphism $Y \rightarrow Z$ [loc. cit., 2.5, 3.1]. As usual we write $\operatorname{Int}(Y)$ in case of the structure morphism $Y \rightarrow \mathscr{M}(L)$. Since $X^{\text {an }}$ is closed we have by definition $\operatorname{Int}\left(X^{\mathrm{an}}\right)=X^{\mathrm{an}}$. Moreover, Proposition 3.1.3(ii) of [loc. cit.] 
implies $\operatorname{Int}(V)=\operatorname{Int}\left(V / X^{\mathrm{an}}\right)$. By part (i) of the same proposition the topological interior of $V$ is equal to $\operatorname{Int}\left(V / X^{\text {an }}\right)$ and, thus, $x \in \operatorname{Int}(V)$. Now the residue field of $L$ being finite, there is a countable basis $\left\{W_{n}\right\}_{n \in \mathbb{N}}$ of neighborhoods of $x$ (see discussion after [loc. cit., 3.2.8]) which consists of strictly affinoid subdomains (even Laurent domains) of $V$ [loc. cit., Proposition 3.2.9]. By smoothness of $X^{\text {an }}$ the local ring $\mathcal{O}_{X^{a n}}{ }_{, x}$ is noetherian regular and hence an integral domain. We may therefore assume that all $W_{n}$ are reduced and irreducible [loc. cit., last sentence of 2.3]. Consider $V_{1}:=W_{n_{1}}$ for some $n_{1} \in \mathbb{N}$. As we have just seen $x \in \operatorname{Int}\left(V_{1}\right)$. Since $\operatorname{Int}\left(V_{1}\right)$ is an open neighborhood of $x$ there is $n_{2}>n_{1}$ such that $W_{n_{2}} \subseteq \operatorname{Int}\left(V_{1}\right)$. We put $V_{2}:=W_{n_{2}}$ and repeat the above argument with $V_{1}$ replaced by $V_{2}$. In this way we find a cofinal sequence $V_{1} \supset V_{2} \supset V_{3} \ldots$ of strictly irreducible reduced affinoid neighborhoods of $x$ with the property $\operatorname{Int}\left(V_{i}\right) \supseteq V_{i+1}$ for all $i \geq 1$. According to [loc. cit., Proposition 2.5.9], the bounded homomorphism of $L$-Banach algebras $\mathcal{A}_{V_{i}} \rightarrow \mathcal{A}_{V_{i+1}}$ associated with the inclusion $V_{i+1} \subset V_{i}$ is inner with respect to $L$ (in the sense of [loc. cit., Definition 2.5.1]. The arguments in [Emerton 2011, Proposition 2.1.16] now show that $\mathcal{A}_{V_{i}} \rightarrow \mathcal{A}_{V_{i+1}}$ is a compact linear map between Banach spaces. Finally, this latter map is injective because $V_{i}$ is irreducible and $V_{i+1}$ contains a nonempty open subset of $V_{i}$. It is also flat since, by construction, $V_{i+1}$ is an affinoid subdomain of $V_{i}$ [Berkovich 1990, Proposition 2.2.4(ii)].

6.2.5. In this paragraph and the next lemma we assume $L=\mathbb{Q}_{p}$. Consider for a given $z \in \mathscr{B}$ the group $U_{z}^{(e)} \subset G$; see Section 4.1.5. For $e \geq e_{\text {uni }}$ the group $U_{z}^{(e)}$ is uniform pro- $p$; see 4.3.4. As such, it has a $\mathbb{Z}_{p}$-Lie algebra $\mathcal{L}\left(U_{z}^{(e)}\right)$, which is powerful, and the exponential map $\exp _{U_{z}^{(e)}}: \mathcal{L}\left(U_{z}^{(e)}\right) \rightarrow U_{z}^{(e)}$ is well-defined and a bijection [Dixon et al. 1999, Section 9.4]. Using the Baker-Campbell-Hausdorff series one can then associate to the lattice $\mathcal{L}\left(U_{z}^{(e)}\right)$ a $\mathbb{Q}_{p}$-analytic affinoid subgroup $\mathbb{U}_{z}^{(e)} \subset \boldsymbol{G}^{\text {an }}$, which has the property that $\mathbb{U}_{z}^{(e)}\left(\mathbb{Q}_{p}\right)=U_{z}^{(e)}{ }^{6}\left(U_{z}^{(e)}\right.$ is a good analytic open subgroup of $G$ in the sense of [Emerton 2011, Section 5.2].) Let $V \subset X^{\text {an }}$ be an affinoid domain. We say that $U_{z}^{(e)}$ acts analytically on $V$, if there is an action of the affinoid group $\mathbb{U}_{z}^{(e)}$ on $V$ compatible with the action of $\mathbb{U}_{z}^{(e)}$ on $X^{\text {an }}$, i.e., if there is a commutative diagram of group operations

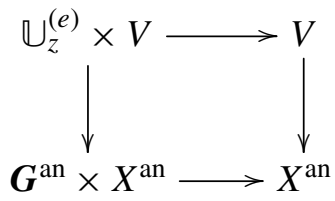

\footnotetext{
${ }^{6}$ Only here do we use that $L=\mathbb{Q} p$. For general $L$ it would be necessary to show that $U_{z}^{(e)}$ is actually an $L$-uniform pro- $p$ group; see [Orlik and Strauch 2010b, 2.2.5]. This can be done, but we do not work here in this generality.
} 
where the vertical maps are inclusions (and the products on the left are taken in the category of $L$-analytic spaces).

Lemma 6.2.6. There exists a number $e_{\mathrm{st}} \geq e_{\mathrm{uni}}$ with the following property. For any point $z \in \mathscr{B}$, viewed as a point in $X^{\text {an }}$, there is a fundamental system of strictly affinoid neighborhoods $\left\{V_{n}\right\}_{n \geq 0}$ of $z$ with the properties as in Lemma 6.2.4, and such that for all $n \geq 0$ and $e \geq e_{\mathrm{st}}$ the group $U_{z}^{(e)}$ acts analytically on $V_{n}$.

The proof, which is lengthy, is given in an appendix, in order not to interrupt the discussion at this point.

\subsection{A structure sheaf on the building.}

6.3.1. To be able to compare the localization of Schneider-Stuhler and BeilinsonBernstein we equip the topological space $\mathscr{B}$ with a sheaf of commutative and topological $L$-algebras. Recall that a subset $V \subset X^{\text {an }}$ is called a special domain if it is a finite union of affinoid domains, and to any special domain $V$ there is associated an $L$-Banach algebra $\mathcal{A}_{V}$ [Berkovich $\left.1990,2.2 .6\right]$. The sheaf $\mathcal{O}_{X}$ an is naturally a sheaf of locally convex algebras as follows: given an open subset $U \subset X^{\text {an }}$ we have

$$
\mathcal{O}_{X} \text { an }(U)=\lim _{V \subset U} \mathcal{A}_{V}
$$

where the limit is taken over all special domains (or affinoid domains) of $X^{\text {an }}$ contained in $U$. Here, $\mathcal{A}_{V}$ is the $L$-Banach algebra corresponding to $V$ and the projective limit is equipped with the projective limit topology. Because the residue field of $L$ is finite, $X^{\text {an }}$ has a countable basis of open subsets [Berkovich 1990, 3.2.9]. Therefore, one can cover $U$ with a countable set of special domains and $\mathcal{O}_{X}$ an $(U)$ is thus a countable projective limit of Banach algebras, hence a Fréchet algebra over $L$.

We then consider the exact functor $\vartheta_{B}^{-1}$ from abelian sheaves on $X^{\text {an }}$ to abelian sheaves on $\mathscr{B}$ given by restriction along $\vartheta_{\boldsymbol{B}}: \mathscr{B} \hookrightarrow X^{\text {an }}$. Let

$$
\mathcal{O}_{\mathscr{B}}:=\vartheta_{\boldsymbol{B}}^{-1}\left(\mathcal{O}_{X} \text { an }\right)
$$

For any subset $C \subset X^{\text {an }}$ we can consider $\mathcal{O}_{X}$ an $(C)$, the vector space of sections of $\mathcal{O}_{X}$ an over $C$, i.e., the global sections of the restriction of the sheaf $\mathcal{O}_{X}$ an to $C$.

Proposition 6.3.2. For any subset $C \subset X^{\text {an }}$ we have

$$
\mathcal{O}_{X} \text { an }(C)=\underset{C \subset U}{\lim _{C}} \mathcal{O}_{X} \text { an }(U)
$$

where $U$ runs through all open neighborhoods of $C$ in $X^{\text {an }}$.

Proof. As was pointed out in 6.3.1, the compact Hausdorff topological space $X^{\text {an }}$ has a countable basis of open subsets. By Urysohn's metrization theorem, it is therefore metrizable, and we may apply [Godement 1958, II.3.3, Corollary 1]. 
In particular, given an open set $\Omega \subseteq \mathscr{B}$ we have

$$
\mathcal{O}_{\mathscr{B}}(\Omega)=\underset{\Omega \subset U}{\lim _{\Omega}} \mathcal{O}_{X} \text { an }(U)
$$

where $U$ runs through the open neighborhoods of $\Omega$ in $X^{\text {an }}$. Using the locally convex inductive limit topology on the right-hand side, the sheaf $\mathcal{O}_{\mathscr{B}}$ becomes in this way a sheaf of locally convex algebras. We point out that the stalk $\mathcal{O}_{\mathscr{B}, z}=\mathcal{O}_{X}{ }^{\text {an }}, z$ for any point $z \in \mathscr{B}$ is in fact a field; see Lemma 6.2.2. We summarize some properties of $\vartheta_{\boldsymbol{B}}^{-1}$ :

(1) $\vartheta_{\boldsymbol{B}}^{-1}$ preserves (commutative) rings, $L$-algebras, $L$-Lie algebras and $G$-equivariance.

(2) $\vartheta_{\boldsymbol{B}}^{-1}$ maps $\mathcal{O}_{X}$ an-modules into $\mathcal{O}_{\mathscr{B}}$-modules.

(3) $\vartheta_{\boldsymbol{B}}^{-1}$ induces a Lie algebra homomorphism $\operatorname{Der}_{L}\left(\mathcal{O}_{X}\right.$ an $) \rightarrow \operatorname{Der}_{L}\left(\mathcal{O}_{\mathscr{B}}\right)$.

(4) $\mathcal{O}_{\mathscr{B}}$ is a sheaf of locally convex $L$-algebras. For every $z \in \mathscr{B}$ the stalk $\mathcal{O}_{\mathscr{B}, z}$ is of compact type with a defining system $\mathcal{A}_{V_{n}}$ of Banach algebras, where $\left(V_{n}\right)_{n}$ is a fundamental system of affinoid neighborhoods as in Lemma 6.2.6.

Composing the map $\mathfrak{g} \rightarrow \operatorname{Der}_{L}\left(\mathcal{O}_{X}\right.$ an $)$ from Lemma 6.1.2 with (3) yields a Lie algebra homomorphism $\mathfrak{g} \rightarrow \operatorname{Der}_{L}\left(\mathcal{O}_{\mathscr{B}}\right)$ and the associated skew enveloping algebra $\mathcal{O}_{\mathscr{B}} \# U(\mathfrak{g})$. By (1),(2) we have the $L$-Lie algebras and $\mathcal{O}_{\mathscr{B}}$-modules $\mathfrak{n}_{\mathscr{B}}^{\circ \text {,an }}:=$ $\vartheta_{\boldsymbol{B}}^{-1}\left(\mathfrak{n}^{\circ, \text { an }}\right)$ and $\mathfrak{b}_{\mathscr{B}}^{\circ, \text { an }}:=\vartheta_{\boldsymbol{B}}^{-1}\left(\mathfrak{b}^{\circ, \text { an }}\right)$. Similarly,

$$
\lambda_{\mathscr{B}}^{\circ, \text { an }}:=\vartheta_{\boldsymbol{B}}^{-1}\left(\lambda^{\circ, \text { an }}\right): \mathfrak{b}_{\mathscr{B}}^{\circ \text {,an }} \rightarrow \mathcal{O}_{\mathscr{B}}
$$

is a morphism of $L$-Lie algebras and $\mathcal{O}_{\mathscr{B}}$-modules. Let $\mathcal{I}_{\mathscr{B}, \mathrm{t}}^{\text {an }}$ resp. $\mathcal{I}_{\mathscr{B}, \chi}^{\text {an }}$ be the right ideal sheaf of $\mathcal{O}_{\mathscr{B}} \# U(\mathfrak{g})$ generated by $\mathfrak{n}_{\mathscr{B}}^{\circ \text { an }} \operatorname{resp} \operatorname{ker}\left(\lambda_{\mathscr{B}}^{\circ \text {,an }}\right)$. One checks that these are two-sided ideals. We let

$$
\mathcal{D}_{\mathscr{B}, \mathfrak{t}}^{\text {an }}:=\left(\mathcal{O}_{\mathscr{B}} \# U(\mathfrak{g})\right) / \mathcal{I}_{\mathscr{B}, \mathfrak{t}}^{\text {an }} \text { and } \mathcal{D}_{\mathscr{B}, \chi}^{\text {an }}:=\left(\mathcal{O}_{\mathscr{B}} \# U(\mathfrak{g})\right) / \mathcal{I}_{\mathscr{B}, \chi}^{\text {an }} .
$$

Note that by exactness of $\vartheta_{\boldsymbol{B}}^{-1}$ we have

$$
\mathcal{D}_{\mathscr{B}, \mathrm{t}}^{\text {an }}=\vartheta_{\boldsymbol{B}}^{-1}\left(\mathcal{D}_{\mathfrak{t}}^{\text {an }}\right) \text { and } \mathcal{D}_{\mathscr{B}, \chi}^{\text {an }}=\vartheta_{\boldsymbol{B}}^{-1}\left(\mathcal{D}_{\chi}^{\mathrm{an}}\right) \text {. }
$$

6.3.3. The sheaf $\mathcal{D}_{\mathscr{B}, \chi}^{\text {an }}$ of twisted differential operators on $\mathscr{B}$ is formed with respect to the Lie algebra action of $\mathfrak{g}$ on the ambient space $\mathscr{B} \subset X^{\text {an }}$. In an attempt to keep track of the whole analytic $G$-action on $X^{\text {an }}$ we will produce in the following a natural injective morphism of sheaves of algebras

$$
\mathcal{D}_{\mathscr{B}, \chi}^{\text {an }} \rightarrow \mathscr{D}_{r, \chi}
$$

with target a sheaf of what we tentatively call twisted distribution operators on $\mathscr{B}$. Actually, there will be one such sheaf for each "radius" $r \in\left[r_{0}, 1\right)$ in $p^{\mathbb{Q}}$ and each 
sufficiently large "level" $e>0$. Again, following [Schneider and Stuhler 1997] we suppress the dependence on the level in our notation.

\subsection{Mahler series and completed skew group rings.}

6.4.1. Suppose for a moment that $\mathcal{A}$ is an arbitrary $L$-Banach algebra. Since $\mathbb{Q}_{p} \subset \mathcal{A}$ the completely valued $\mathbb{Z}_{p}$-module $(\mathcal{A},|\cdot|)$ is saturated in the sense of [Lazard 1965, I.2.2.10]. Consequently, we have the theory of Mahler expansions over $\mathcal{A}$ at our disposal [loc. cit., III.1.2.4 and III.1.3.9]. In this situation we prove a version of the well-known relation between decay of Mahler coefficients and overconvergence.

Proposition 6.4.2. Let $f=\sum_{\alpha \in \mathbb{N}_{0}^{d}} a_{\alpha} x^{\alpha}$ be a d-variable power series over $\mathcal{A}$ converging on the disc $\left|x_{i}\right| \leq R$ for some $R>1$. Let $c>0$ be a constant such that $\left|a_{\alpha}\right| \leq c R^{-|\alpha|}$ for all $\alpha$. Let

$$
f(\cdot)=\sum_{\alpha \in \mathbb{N}_{0}^{d}} c_{\alpha}\left(\begin{array}{c}
\cdot \\
\alpha
\end{array}\right),
$$

$c_{\alpha} \in \mathcal{A}$, be the Mahler series expansion of $f$. Then $\left|c_{\alpha}\right| \leq c s^{|\alpha|}$ for all $\alpha$, where $s=\tilde{r} R^{-1}$ with $\tilde{r}=p^{-1 /(p-1)}$.

Proof. We prove the lemma in case $d=1$. The general case follows along the same lines but with more notation. We define the following series of polynomials over $\mathbb{Z}$

$$
(x)_{0}=1, \ldots,(x)_{k}=x(x-1) \cdots(x-k+1)
$$

for $k \geq 1$. The $\mathbb{Z}$-module $\mathbb{Z}[x]$ has the $\mathbb{Z}$-bases $\left\{x^{k}\right\}_{k \geq 0}$ and $\left\{(x)_{k}\right\}_{k \geq 0}$ and the transition matrices are unipotent upper triangular. We may therefore write

$$
x^{n}=\sum_{k=0, \ldots, n} s(n, k)(x)_{k}
$$

with $s(n, k) \in \mathbb{Z}$. Put $b_{k}:=c_{k} / k$ !. Then

$$
\sum_{k \geq 0} c_{k}\left(\begin{array}{l}
x \\
k
\end{array}\right)=\sum_{k \geq 0} b_{\kappa}(x)_{k}
$$

is a uniform limit of continuous functions (even polynomials) on $\mathbb{Z}_{p}$ [Robert 2000, Theorem VI.4.7]. We now proceed as in (the proof of) [Washington 1997, Proposition 5.8]. Fix $i \geq 1$ and write

$$
\sum_{n \leq i} a_{n} x^{n}=\sum_{k \leq i} b_{k, i}(x)_{k}
$$

as polynomials over $\mathcal{A}$ with some elements $b_{k, i} \in \mathcal{A}$. Inserting (6.4.3) and comparing coefficients yields $b_{k, i}=\sum_{k \leq n \leq i} a_{n} s(n, k)$ and consequently,

$$
\left|b_{k, i}\right| \leq \max _{k \leq n \leq i}\left|a_{n}\right| \leq \max _{k \leq n \leq i}\left(c R^{-n}\right) \leq c R^{-k}
$$


since $R^{-1}<1$. It follows that, for $j \geq i$, we have

$$
\left|b_{k, j}-b_{k, i}\right|=\left|\sum_{n=i+1}^{j} a_{n} s(n, k)\right| \leq R^{-(i+1)} .
$$

We easily deduce from this that $\left\{b_{k, i}\right\}_{i \geq 0}$ is a Cauchy sequence in the Banach space $\mathcal{A}$. Let $\tilde{b}_{k}$ be its limit. Clearly, $\left|\tilde{b}_{k}\right| \leq c R^{-k}$. Put $\tilde{c}_{k}:=k ! \tilde{b}_{k}$. Since $|k !| \leq \tilde{r}^{k}$ we obtain $\left|\tilde{c}_{k}\right| \leq c\left(\tilde{r} R^{-1}\right)^{k}=c s^{k}$ for all $k$. By definition of $\tilde{b}_{k}$ the series of polynomials

$$
\sum_{k \geq 0} \tilde{c}_{k}\left(\begin{array}{l}
x \\
k
\end{array}\right)=\sum_{k \geq 0} \tilde{b}_{\kappa}(x)_{k}
$$

converges pointwise to the limit

$$
\lim _{i \rightarrow \infty} \sum_{k \leq i} b_{k, i}(x)_{k}=\lim _{i \rightarrow \infty} \sum_{n \leq i} a_{n} x^{n}=f(x) .
$$

By [Robert 2000, IV.2.3, p. 173] this convergence is uniform and so uniqueness of Mahler expansions implies $\tilde{c}_{k}=c_{k}$ for all $k$. This proves the lemma.

Corollary 6.4.4. Let $L=\mathbb{Q}_{p}, z \in \mathscr{B}$, and $e>e_{\text {uni }}$.

(i) Consider an affinoid domain $V$ of $X^{\text {an }}$ on which $U_{z}^{(e-1)}$ acts analytically in the sense of Section 6.2.5, and let $\mathcal{A}_{V}$ be the corresponding Banach algebra.

(a) For any p-basis $\left(h_{1}, \ldots, h_{d}\right)$ of $U_{z}^{(e-1)}$ (see Section 2.2), and for any $f \in \mathcal{A}_{V}$ the orbit map $U^{(e-1)} \rightarrow \mathcal{A}_{V}, h=h_{1}^{x_{1}} \cdots h_{d}^{x_{d}} \mapsto h . f$, can be expanded as a strictly convergent power series $\sum_{v \in \mathbb{N}^{d}} f_{\nu} x_{1}^{\nu_{1}} \cdots x_{d}^{\nu_{d}}$ with $f_{v} \in \mathcal{A}_{V}$ and $\left|f_{v}\right|_{V} \rightarrow 0$ as $|v| \rightarrow \infty$. $\left(|\cdot|_{V}\right.$ denotes the supremum norm on $\left.\mathcal{A}_{V}.\right)$

(b) The representation $\rho: U_{z}^{(e)} \rightarrow \operatorname{GL}\left(\mathcal{A}_{V}\right),(\rho(h) . f)(w)=f\left(h^{-1} \cdot w\right)$, satisfies the assumption $(\star)$ of Section 3.2.5 for any $r \in\left[r_{0}, 1\right)$. In particular, the ring

$$
\mathcal{A}_{V} \# D_{r}\left(U_{z}^{(e)}, K\right)
$$

exists for all $r \in\left[r_{0}, 1\right)$.

(ii) More generally, let $V=V_{1} \cup \ldots \cup V_{m}$ be a special domain of $X^{\text {an }}$ (see 6.3.1), where $V_{i}$ is affinoid for $1 \leq i \leq m$, and suppose that $U^{(e-1)}$ acts analytically on each $V_{i}, 1 \leq i \leq m$. Then the representation $\rho: U_{z}^{(e)} \rightarrow \operatorname{GL}\left(\mathcal{A}_{V}\right),(\rho(h) . f)(w)=$ $f\left(h^{-1} . w\right)$, satisfies the assumption $(\star)$ of Section 3.2.5 for any $r \in\left[r_{0}, 1\right)$. In particular, the ring

$$
\mathcal{A}_{V} \# D_{r}\left(U_{z}^{(e)}, K\right)
$$

exists for all $r \in\left[r_{0}, 1\right)$.

Proof. (i)(a) To simplify notation put $U=U_{z}^{(e-1)}$ and $\mathbb{U}=\mathbb{U}_{z}^{(e-1)}$; see Section 6.2.5. Let $\mathcal{A}_{\mathbb{U}}$ be the affinoid algebra of $\mathbb{U}$. The $p$-basis gives rise to an isomorphism 
$\mathcal{A}_{\mathbb{U}} \simeq \mathbb{Q}_{p}\left\langle x_{1}, \ldots, x_{d}\right\rangle$, where the latter denotes strictly convergent power series. The action of $\mathbb{U}$ on $V$ corresponds to a morphism of affinoid algebras

$$
\mathcal{A}_{V} \rightarrow \mathcal{A}_{\mathbb{U}} \hat{\otimes}_{L} \mathcal{A}_{V} \simeq \mathcal{A}_{V}\left\langle x_{1}, \ldots, x_{d}\right\rangle .
$$

On the right we have the algebra of strictly convergent power series over $\mathcal{A}_{V}$. This proves the first assertion.

(i)(b) By 4.3 .4 we have that $U_{z}^{(e)}$ is the second member of the lower $p$-series of $U_{z}^{(e-1)}$. Therefore, if $\left(h_{1}, \ldots, h_{d}\right)$ is the $p$-basis for $U_{z}^{(e-1)}$ used in (i), it follows that $\left(h_{1}^{p}, \ldots, h_{d}^{p}\right)$ is a $p$-basis for $U_{z}^{(e)}$. Denote by $\left(y_{1}, \ldots, y_{d}\right)$ the coordinates on $U_{z}^{(e)}$ corresponding to this $p$-basis. Then, applying (i) to the group $U_{z}^{(e)}$, we find

$$
\rho(h) . f=\sum_{\nu \in \mathbb{N}^{d}} f_{\nu} y_{1}^{\nu_{1}} \cdots y_{d}^{v_{d}}
$$

when $h=\left(h_{1}^{p}\right)^{y_{1}} \cdots\left(h_{d}^{p}\right)^{y_{d}} \in U_{z}^{(e)}$ and $f \in \mathcal{A}_{V}$. Therefore, the right-hand side of (6.4.5) converges on the disc $\left|y_{i}\right| \leq p$. Next consider the Mahler expansion

$$
\rho\left(\left(h_{1}^{p}\right)^{y_{1}} \cdots\left(h_{d}^{p}\right)^{y_{d}}\right) \cdot f=\sum_{\alpha \in \mathbb{N}^{d}} c_{f, \alpha}\left(\begin{array}{c}
y \\
\alpha
\end{array}\right) .
$$

By Proposition 6.4.2 we have $\left|c_{f, \alpha}\right|_{V} \leq c s^{|\alpha|}$ with some $c>0$ and $s=r_{1} p^{-1}<$ $p^{-1}=r_{0}$.

Write $\delta \in D_{r}\left(U_{z}^{(e)}, L\right)$ as a series $\delta=\sum_{\alpha \in \mathbb{N}^{d}} d_{\alpha} \boldsymbol{b}^{\alpha}$ with $\boldsymbol{b}^{\alpha}=\left(h_{1}^{p}-1\right)^{\alpha_{1}} \ldots$ $\cdot\left(h_{d}^{p}-1\right)^{\alpha_{d}}$ and $d_{\alpha} \in L$ such that $\left|d_{\alpha}\right| r^{|\alpha|} \rightarrow 0$. Since $s<r_{0} \leq r$ and $\left|c_{f, \alpha}\right|_{V} \leq c s^{|\alpha|}$ the sum

$$
\delta . f=\delta(h \mapsto \rho(h) . f)=\sum_{\alpha \in \mathbb{N}^{d}} d_{\alpha} c_{f, \alpha}
$$

converges in the Banach space $\mathcal{A}_{V}$. The map $(\delta, f) \mapsto \delta . f$ makes $\mathcal{A}_{V}$ a topological module over $D_{r}\left(U_{z}^{(e)}, L\right)$ in a way compatible with the map $D\left(U_{z}^{(e)}\right) \rightarrow D_{r}\left(U_{z}^{(e)}\right)$. The last assertion is contained in Section 3.2.5.

(ii) As $X$ is separated, $X^{\text {an }}$ is Hausdorff and therefore separated [Berkovich 1990, 3.4.8 and 3.1.5]. This implies that the intersection of any two affinoid domains in $X^{\text {an }}$ is again an affinoid domain [loc. cit., 3.1.6]. In this case,

$$
\mathcal{A}_{V}=\operatorname{ker}\left(\prod_{i=1}^{m} \mathcal{A}_{V_{i}} \rightrightarrows \prod_{i, j} \mathcal{A}_{V_{i} \cap V_{j}}\right) ;
$$

see [loc. cit., 2.2.6 and 3.3]. We can now apply the assertions in (i)(b) to each factor $\mathcal{A}_{V_{i}}$ and $\mathcal{A}_{V_{i} \cap V_{j}}$, and to the corresponding products, and deduce statement (ii). 
6.4.7. Later on we will sometimes need to consider the action of $U_{z}^{(e)}$ on rings of the form $\mathcal{A}_{V}$, where $V$ is a special domain in $X^{\text {an }}$ as in Corollary 6.4.4(ii). In order to conveniently refer to this situation, we will say that $U_{z}^{(e)}$ acts analytically on a special domain $V$, if one can write $V=V_{1} \cup \ldots \cup V_{m}$ as finite union of affinoid domains $V_{i} \subset X^{\text {an }}, 1 \leq i \leq m$, with the property that $U_{z}^{(e)}$ acts analytically on each $V_{i}, 1 \leq i \leq m$.

Until the end of this section we will assume $L=\mathbb{Q}_{p}, e>e_{\mathrm{st}}$ and $r \in\left[r_{0}, 1\right)$.

Proposition 6.4.8. Let $z \in \mathscr{B}$, and let $\left(V_{n}\right)_{n}$ be a descending sequence of affinoid neighborhoods of $z$ as in Lemma 6.2.6. Then the stalk $\mathcal{O}_{\mathscr{B}, z}$ is equal to the inductive limit of the Banach algebras $\mathcal{A}_{V_{n}}$, the completed skew group rings $\mathcal{A}_{V_{n}} \# D_{r}\left(U_{z}^{(e)}, K\right)$ and $\mathcal{O}_{\mathscr{B}, z} \# D_{r}\left(U_{z}^{(e)}, K\right)$ exist, and the natural map

$$
\underset{n}{\lim }\left(\mathcal{A}_{V_{n}} \# D_{r}\left(U_{z}^{(e)}, K\right)\right) \rightarrow \mathcal{O}_{\mathscr{B}, z} \# D_{r}\left(U_{z}^{(e)}, K\right)
$$

is an isomorphism of $K$-algebras.

Proof. By Lemmas 6.2.4 and 6.2.6, the stalk $\mathcal{O}_{\mathscr{B}, z}$ is the inductive limit of the Banach algebras $\mathcal{A}_{V_{n}}$, and the transition maps $\mathcal{A}_{V_{n}} \rightarrow \mathcal{A}_{V_{n+1}}$ are compact and injective. By Proposition 2.4.1, the natural map

$\stackrel{\lim }{\longrightarrow}\left(\mathcal{A}_{V_{n}} \hat{\otimes}_{L} D_{r}\left(U_{z}^{(e)}, K\right)\right) \rightarrow$

$$
\left(\stackrel{\lim }{\longrightarrow} \mathcal{A}_{V_{n}}\right) \hat{\otimes}_{L} D_{r}\left(U_{z}^{(e)}, K\right)=\mathcal{O}_{\mathscr{B}, z} \hat{\otimes}_{L} D_{r}\left(U_{z}^{(e)}, K\right)
$$

is an isomorphism of vector spaces. By Corollary 6.4.4(i)(b) the ring $\mathcal{A}_{V_{n}}$ is a $D_{r}\left(U_{z}^{(e)}, L\right)$-module for every $n$ and $r \in\left[r_{0}, 1\right)$, and the transition maps are homomorphisms of $D_{r}\left(U_{z}^{(e)}, L\right)$-modules. This shows that $\mathcal{O}_{\mathscr{B}, z}$ is naturally a $D_{r}\left(U_{z}^{(e)}, L\right)$-module, hence $\mathcal{O}_{\mathscr{B}, z} \# D_{r}\left(U_{z}^{(e)}, L\right)$ and $\mathcal{O}_{\mathscr{B}, z} \# D_{r}\left(U_{z}^{(e)}, K\right)$ exist. The natural map $\mathcal{A}_{V_{n}} \# D_{r}\left(U_{z}^{(e)}, K\right) \rightarrow \mathcal{O}_{\mathscr{B}, z} \# D_{r}\left(U_{z}^{(e)}, K\right)$ is a ring homomorphism, and the map (6.4.9) is an isomorphism of $K$-algebras.

The following corollary is immediate and recorded only for future reference.

Corollary 6.4.10. Let $V$ be a neighborhood of $z$ which is a special subset of $X^{\text {an }}$. Suppose $U^{(e-1)}$ acts analytically on $V$ in the sense of Section 6.4.7. Let $\iota_{z}$ be the natural map $\mathcal{A}_{V} \rightarrow \mathcal{O}_{\mathscr{B}, z}$ sending a function to its germ at $z$. The map $\iota_{z} \hat{\otimes} \mathrm{id}$ is an algebra homomorphism

$$
\mathcal{A}_{V} \# D_{r}\left(U_{z}^{(e)}, K\right) \rightarrow \mathcal{O}_{\mathscr{B}, z} \# D_{r}\left(U_{z}^{(e)}, K\right) .
$$

Corollary 6.4.11. Let $V$ be a neighborhood of $z$ which is a special subset of $X^{\text {an }}$. Suppose $U^{(e-1)}$ acts analytically on $V$ in the sense of Section 6.4.7. Then the inclusions

$$
L\left[U_{z}^{(e)}\right] \subseteq D_{r}\left(U_{z}^{(e)}, K\right)
$$


and $U(\mathfrak{g})_{K} \subseteq D_{r}\left(U_{z}^{(e)}, K\right)$ induce algebra homomorphisms

(i) $\mathcal{A}_{V} \# U_{z}^{(e)}=\mathcal{A}_{V} \otimes_{L} L\left[U_{z}^{(e)}\right] \rightarrow \mathcal{A}_{V} \# D_{r}\left(U_{z}^{(e)}, K\right)$,

(ii) $\mathcal{A}_{V} \# U(\mathfrak{g})_{K} \rightarrow \mathcal{A}_{V} \# D_{r}\left(U_{z}^{(e)}, K\right)$.

If $V$ runs through a sequence of affinoid neighborhoods of $z$ as in Lemma 6.2.6 these maps assemble to algebra homomorphisms

(i) $\mathcal{O}_{\mathscr{B}, z} \# U_{z}^{(e)}=\mathcal{O}_{\mathscr{B}, z} \otimes_{L} L\left[U_{z}^{(e)}\right] \rightarrow \mathcal{O}_{\mathscr{B}, z} \# D_{r}\left(U_{z}^{(e)}, K\right)$,

(ii) $\mathcal{O}_{\mathscr{B}, z} \# U(\mathfrak{g})_{K} \rightarrow \mathcal{O}_{\mathscr{B}, z} \# D_{r}\left(U_{z}^{(e)}, K\right)$.

Proof. Consider the case of $\mathcal{A}_{V}$. The existence of the map (i) follows from Proposition 3.2.4. The same is true for the map (ii) once we convince ourselves that there is a commutative diagram of algebra homomorphisms

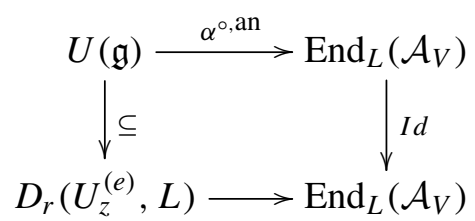

where the upper horizontal arrow is derived from (6.1.3) and the lower horizontal arrow describes the $D_{r}\left(U_{z}^{(e)}, L\right)$-module structure of $\mathcal{A}_{V}$ as given by Corollary 6.4.4. Restricting the lower horizontal arrow to $\mathfrak{g}$ amounts to differentiating the analytic $U_{z}^{(e)}$-action on $\mathcal{A}_{V}$. This action comes from the algebraic action of $\boldsymbol{G}$ on $X$. The diagram commutes by the remark following Lemma 6.1.2. Having settled the case $\mathcal{A}_{V}$ the case of $\mathcal{O}_{\mathscr{B}, z}$ now follows by passage to the inductive limit.

As a result of this discussion we have associated to each point $z \in \mathscr{B} \subset X^{\text {an }}$ the (noncommutative) $K$-algebra $\mathcal{O}_{\mathscr{B}, z} \# D_{r}\left(U_{z}^{(e)}, K\right)$. As we have seen, it comes together with an injective algebra homomorphism

$$
\mathcal{O}_{\mathscr{B}, z} \# U(\mathfrak{g})_{K} \hookrightarrow \mathcal{O}_{\mathscr{B}, z} \# D_{r}\left(U_{z}^{(e)}, K\right) .
$$

In the next section we will sheafify this situation and obtain a sheaf of noncommutative $K$-algebras $\mathcal{O}_{\mathscr{B}} \# D_{r}$ on $\mathscr{B}$ together with an injective morphism of sheaves of algebras

$$
\mathcal{O}_{\mathscr{B}} \# U(\mathfrak{g})_{K} \hookrightarrow \mathcal{O}_{\mathscr{B}} \# D_{r}
$$

inducing the map (6.4.12) at all points $z \in \mathscr{B}$. To do this we shall need a simple "gluing property" of the algebras $\mathcal{O}_{\mathscr{B}, z} \# D_{r}\left(U_{z}^{(e)}, K\right)$.

Lemma 6.4.13. Let $F, F^{\prime}$ be facets in $\mathscr{B}$ such that $F^{\prime} \subseteq \bar{F}$ and let

$$
\sigma_{r}^{F^{\prime} F}: D_{r}\left(U_{F^{\prime}}^{(e)}, K\right) \rightarrow D_{r}\left(U_{F}^{(e)}, K\right)
$$


be the corresponding algebra homomorphism. Suppose $V$ and $V^{\prime}$ are two special domains in $X^{\text {an }}$ on which $U_{F}^{(e-1)}$ and $U_{F^{\prime}}^{(e-1)}$, respectively, act analytically (see Section 6.4.7). If $V \subset V^{\prime}$ the map $\operatorname{res}_{V}^{V^{\prime}} \hat{\otimes} \sigma_{r}^{F^{\prime} F}$ is a continuous algebra homomorphism,

$$
\operatorname{res}_{V}^{V^{\prime}} \hat{\otimes} \sigma_{r}^{F^{\prime} F}: \mathcal{A}_{V^{\prime}} \# D_{r}\left(U_{F^{\prime}}^{(e)}, K\right) \rightarrow \mathcal{A}_{V} \# D_{r}\left(U_{F}^{(e)}, K\right) .
$$

Proof. Since the map $\sigma_{r}^{F^{\prime} F}$ is induced from the inclusion $U_{F^{\prime}}^{(e)} \subseteq U_{F}^{(e)}$ there is a commutative diagram

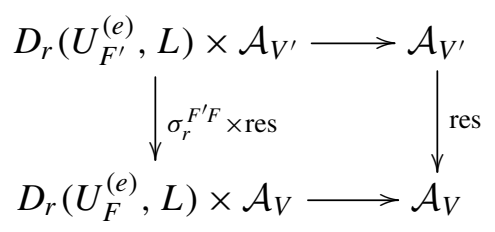

where the horizontal arrows describe the module structures of $\mathcal{A}_{V^{\prime}}$ and $\mathcal{A}_{V}$ over $D_{r}\left(U_{F^{\prime}}^{(e)}, L\right)$ and $D_{r}\left(U_{F}^{(e)}, L\right)$ respectively; see Corollary 6.4.4. The assertion follows now from the construction of the skew multiplication of the source and target of $\operatorname{res}_{V}^{V^{\prime}} \hat{\otimes} \sigma_{r}^{F^{\prime} F}$ (see Section 3).

6.4.14. For any subset $C \subset X^{\text {an }}$ we have by Proposition 6.3 .2

$$
\mathcal{O}_{X} \text { an }(C)=\underset{U}{\lim } \mathcal{O}_{X} \operatorname{an}(U),
$$

where $U$ runs over all open neighborhoods of $C$ in $X^{\text {an }}$. Obviously, if $C$ is contained in $\mathscr{B}$ we have $\mathcal{O}_{\mathscr{B}}(C)=\mathcal{O}_{X}$ an $(C)$. We recall that the star of a facet $F^{\prime}$ in $\mathscr{B}$ is the subset of $\mathscr{B}$ defined by

$$
\operatorname{St}(F):=\text { union of all facets } F^{\prime} \subseteq \mathscr{B} \text { such that } F \subseteq \overline{F^{\prime}} .
$$

These stars form a locally finite open covering of $\mathscr{B}$.

Proposition 6.4.15. Let $F \subset \mathscr{B}$ be a facet, and let $C \subset \operatorname{St}(F)$ be a compact set.

(i) There is a countable fundamental system of neighborhoods $V_{1} \supset V_{2} \supset \cdots$ of $C$ in $X^{\text {an }}$ with the following properties:

- For all $i$ the neighborhood $V_{i}$ is a special subdomain on which $U_{F}^{(e)}$ acts analytically.

- For all $i<j$ the induced map $\mathcal{A}_{V_{i}} \rightarrow \mathcal{A}_{V_{j}}$ is compact and injective.

(ii) Let $\left(V_{i}\right)_{i}$ be as in (i). Then the rings $\mathcal{A}_{V_{i}} \# D_{r}\left(U_{F}^{(e)}\right)$ exist for all $i$ and the maps

$$
\mathcal{A}_{V_{i}} \# D_{r}\left(U_{F}^{(e)}\right) \rightarrow \mathcal{A}_{V_{i+1}} \# D_{r}\left(U_{F}^{(e)}\right)
$$

induced by the restriction maps $\mathcal{A}_{V_{i}} \rightarrow \mathcal{A}_{V_{i+1}}$ are homomorphisms of $K$-algebras. 
(iii) Let $\left(V_{i}\right)_{i}$ be as in (i). Then the maps

$$
\mathcal{A}_{V_{i}} \hat{\otimes}_{L} D_{r}\left(U_{F}^{(e)}\right) \rightarrow \mathcal{O}_{\mathscr{B}}(C) \hat{\otimes}_{L} D_{r}\left(U_{F}^{(e)}\right)
$$

induced by the canonical maps $\mathcal{A}_{V_{i}} \rightarrow \mathcal{O}_{\mathscr{B}}(C)$ induce an isomorphism of vector spaces

$$
\underset{i}{\lim }\left(\mathcal{A}_{V_{i}} \hat{\otimes}_{L} D_{r}\left(U_{F}^{(e)}\right)\right) \rightarrow \mathcal{O}_{\mathscr{B}}(C) \hat{\otimes}_{L} D_{r}\left(U_{F}^{(e)}\right) .
$$

(iv) The left-hand side of (6.4.16) carries a unique structure of a $K$-algebra, such that the canonical maps

$$
\mathcal{A}_{V_{i}} \hat{\otimes}_{L} D_{r}\left(U_{F}^{(e)}\right) \rightarrow \underset{i}{\lim }\left(\mathcal{A}_{V_{i}} \hat{\otimes}_{L} D_{r}\left(U_{F}^{(e)}\right)\right)
$$

become $K$-algebra homomorphisms. Consequently, via transport of structure, we give $\mathcal{O}_{\mathscr{B}}(C) \hat{\otimes}_{L} D_{r}\left(U_{F}^{(e)}\right)$ the unique $K$-algebra structure, henceforth denoted by $\mathcal{O}_{\mathscr{B}}(C) \# D_{r}\left(U_{F}^{(e)}\right)$, such that (6.4.16) becomes an isomorphism of $K$-algebras.

Proof. (i) By [Berkovich 1990, 3.2.9] there is a countable fundamental system of open neighborhoods $W_{1} \supset W_{2} \supset \cdots$ of $C$ in $X^{\text {an }}$. We are going to find inductively the special domain $V_{i} \subset W_{i}$. To begin, use Lemma 6.2.6 to find for each $x \in C$ an affinoid neighborhood $W_{1, x} \subset W_{1}$ on which $U_{F}^{(e)}$ acts analytically. Clearly, we may furthermore assume that every $W_{1, x}$ is connected. Denote by $\operatorname{Int}\left(W_{1, x}\right)$ the topological interior of $W_{1, x}$. As $C$ is compact it is contained in a finite union $\operatorname{Int}\left(W_{1, x_{1}}\right) \cup \ldots \cup \operatorname{Int}\left(W_{1, x_{m_{1}}}\right)$. Put

$$
V_{1}=W_{1, x_{1}} \cup \ldots \cup W_{1, x_{m_{1}}} .
$$

Now suppose we have found a special domain $V_{i}=W_{i, z_{1}} \cup \ldots \cup W_{i, z_{m_{i}}}$ contained in $W_{i}$ with the property that for all $1 \leq j \leq m_{i}$

- $W_{i, z_{j}}$ is a connected affinoid neighborhood of $z_{i}$ on which $U_{F}^{(e)}$ acts analytically, for all $1 \leq j \leq m_{i}$,

- $C$ is contained in the union of the $\operatorname{Int}\left(W_{i, z_{j}}\right)$, for $1 \leq j \leq m_{i}$.

For a given $z^{\prime} \in C$ choose $j\left(z^{\prime}\right) \in\left\{1, \ldots, m_{i}\right\}$ such that $z^{\prime}$ is contained in $\operatorname{Int}\left(W_{i, z_{j\left(z^{\prime}\right)}}\right)$. Use again Lemma 6.2.6 to find a connected affinoid neighborhood $W_{i+1, z^{\prime}}$ of $z^{\prime}$ contained in $W_{i+1} \cap \operatorname{Int}\left(W_{i, z_{j\left(z^{\prime}\right)}}\right)$ on which $U_{F}^{(e)}$ acts analytically. Put $z=z_{j\left(z^{\prime}\right)}$. As $W_{i, z}$ is connected, it is irreducible [loc. cit., 3.1.8], and so is $\operatorname{Spec}\left(\mathcal{A}_{W_{i, z}}\right)$. The ring $\mathcal{A}_{W_{i, z}}$ is hence an integral domain, and the restriction map

$$
\mathcal{A}_{W_{i, z}} \rightarrow \mathcal{A}_{W_{i+1, z^{\prime}}}
$$

is thus injective. Moreover, since $W_{i+1, z^{\prime}}$ is contained in $\operatorname{Int}\left(W_{i, z}\right)$, the map (6.4.17) is inner, by [loc. cit., 2.5.9]. The arguments in [Emerton 2011, 2.1.16] then show that (6.4.17) is a compact map of Banach spaces. Now choose $z_{1}^{\prime}, \ldots, z_{m_{i+1}}^{\prime}$ such 
that $C$ is contained in the union of the $\operatorname{Int}\left(W_{i+1, z_{j}^{\prime}}\right)$, for $1 \leq j \leq m_{i+1}$, and let $V_{i+1}$ be the union of the $W_{i+1, z_{j}^{\prime}}$, for $1 \leq j \leq m_{i+1}$. Recall that

$$
\mathcal{A}_{V_{i}}=\operatorname{ker}\left(\prod_{j=1}^{m_{i}} \mathcal{A}_{W_{i, z_{j}}} \rightrightarrows \prod_{j, j^{\prime}} \mathcal{A}_{W_{i, z_{j}} \cap W_{i, z_{j^{\prime}}}}\right) ;
$$

see [Berkovich 1990, 2.2.6 and 3.3]. Consider the commutative diagram

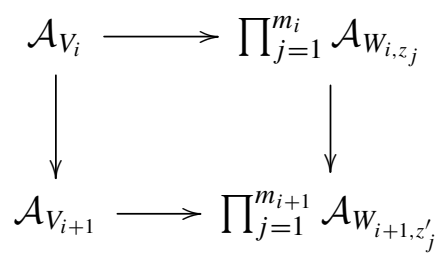

The horizontal arrows are the obvious inclusions. The vertical arrow on the right is the one induced by the maps (6.4.17), and is thus injective and compact. The canonical vertical arrow on the left is thus injective and compact too [Schneider 2002, 16.7 (ii)]. This proves the first assertion.

(ii) This follows immediately from the second part of Corollary 6.4.4, together with Lemma 6.4.13.

(iii) Because $\left(V_{i}\right)_{i}$ is a fundamental system of neighborhoods of $C$ we have, by Proposition 6.3.2, $\mathcal{O}_{\mathscr{B}}(C)=\lim _{\rightarrow} \mathcal{A}_{V_{i}}$. The assertion now follows from (i) and Proposition 2.4.1.

(iv) Using (ii) we see that the right-hand side of (6.4.16) has a canonical $K$-algebra structure. The remaining assertions are now clear.

\section{A sheaf of "distribution operators" on the building}

In this section we assume throughout $L=\mathbb{Q}_{p}, e>e_{\text {st }}$ (compare Lemma 6.2.6), and $r \in\left[r_{0}, 1\right)$. Because $e-1 \geq e_{\mathrm{st}} \geq e_{\text {uni }}$ (see again Lemma 6.2.6), all groups $U_{z}^{(e-1)}$ are uniform pro- $p$ groups; see Remark 4.3.4. We will work from now on exclusively over the coefficient field $K$. To ease notation we will therefore drop this coefficient field from the notation when working with distribution algebras. We thus write $D(G)=D(G, K), D_{r}\left(U_{F}^{(e)}\right)=D_{r}\left(U_{F}^{(e)}, K\right)$ etc.

Recall that the sheaf of (twisted) differential operators $\mathcal{D}_{\chi}$ on $X$ may be constructed from the skew tensor product $\mathcal{O}_{X} \# U(\mathfrak{g})$; see Section 5. In a similar way we are going to construct a sheaf of "distribution operators" on $\mathscr{B}$ starting from a twisted tensor product $\mathcal{O}_{\mathscr{B}} \# \underline{D}_{r}$. Here, $\underline{D}_{r}$ replaces the constant sheaf $U(\mathfrak{g})$ and equals a sheaf of distribution algebras on $\mathscr{B}$. It will be a constructible sheaf with respect to the usual polysimplicial structure of $\mathscr{B}$. Recall from Section 4.2 that a 
sheaf on a polysimplicial space is called constructible if its restriction to a given geometric polysimplex is a constant sheaf.

7.1. A constructible sheaf of distribution algebras. Given an open set $\Omega \subset \mathscr{B}$ we have for any $z \in \Omega$ the natural map

$$
\iota_{z}: \mathcal{O}_{\mathscr{B}}(\Omega) \rightarrow \mathcal{O}_{\mathscr{B}, z}, \quad f \mapsto \text { germ of } f \text { at } z .
$$

Definition 7.1.1. For an open subset $\Omega \subseteq \mathscr{B}$ let

$$
\underline{D}_{r}(\Omega):=K \text {-vector space of all maps } s: \Omega \rightarrow \bigcup_{z \in \Omega} D_{r}\left(U_{z}^{(e)}\right) \text { such that }
$$

(1) $s(z) \in D_{r}\left(U_{z}^{(e)}\right)$ for all $z \in \Omega$, and

(2) for each facet $F \subseteq \mathscr{B}$ there exists a finite open covering $\Omega \cap \operatorname{St}(F)=\bigcup_{i \in I} \Omega_{i}$ with the property: for each $i$ with $\Omega_{i} \cap F \neq \varnothing$ there is an element $s_{i} \in D_{r}\left(U_{F}^{(e)}\right)$ satisfying the following conditions:

(a) $s(z)=s_{i}$ for any $z \in \Omega_{i} \cap F$.

(b) $s\left(z^{\prime}\right)=\sigma_{r}^{F F^{\prime}}\left(s_{i}\right)$ for any $z^{\prime} \in \Omega_{i}$. Here, $F^{\prime}$ is the unique facet in $\operatorname{St}(F)$ that contains $z^{\prime}$.

From (a) it is easy to see that the restriction of $\underline{D}_{r}$ to a facet $F$ is the constant sheaf with value $D_{r}\left(U_{F}^{(e)}\right)$. Hence $\underline{D}_{r}$ is constructible. Furthermore, if $\Omega^{\prime} \subseteq \Omega$ is an open subset there is the obvious restriction map $\underline{D}_{r}(\Omega) \rightarrow \underline{D}_{r}\left(\Omega^{\prime}\right)$. The proof of the following result is implicitly contained in the proofs of Lemmas 7.2.2 and 7.2.3 below.

Lemma 7.1.2. With pointwise multiplication $\underline{D}_{r}$ is a sheaf of $K$-algebras. For $z \in \mathscr{B}$ one has $\left(\underline{D}_{r}\right)_{z}=D_{r}\left(U_{z}^{(e)}\right)$.

\subsection{Sheaves of completed skew group rings.}

Definition 7.2.1. For an open subset $\Omega \subseteq \mathscr{B}$ let

$\left(\mathcal{O}_{\mathscr{B}} \# \underline{D}_{r}\right)(\Omega):=K$-vector space of all maps $s: \Omega \rightarrow \bigcup_{z \in \Omega}^{\cdot} \mathcal{O}_{\mathscr{B}, z} \# D_{r}\left(U_{z}^{(e)}\right)$ such that

(1) $s(z) \in \mathcal{O}_{\mathscr{B}, z} \# D_{r}\left(U_{z}^{(e)}\right)$ for all $z \in \Omega$, and

(2) for each facet $F \subseteq \mathscr{B}$ there exists a finite open covering $\Omega \cap \operatorname{St}(F)=\bigcup_{i \in I} \Omega_{i}$ with the property that, for each $i$ with $\Omega_{i} \cap F \neq \varnothing$, there exists

$$
s_{i} \in \mathcal{O}_{\mathscr{B}}\left(\Omega_{i}\right) \hat{\otimes}_{L} D_{r}\left(U_{F}^{(e)}\right)
$$

satisfying the following conditions:

(a) $s(z)=\left(\iota_{z} \hat{\otimes}\right.$ id $)\left(s_{i}\right)$ for any $z \in \Omega_{i} \cap F$.

(b) $s\left(z^{\prime}\right)=\left(\iota_{z^{\prime}} \hat{\otimes} \sigma_{r}^{F F^{\prime}}\right)\left(s_{i}\right)$ for any $z^{\prime} \in \Omega_{i}$. Here, $F^{\prime}$ is the unique facet in $\operatorname{St}(F)$ that contains $z^{\prime}$. 
Consider a map $s: \Omega \rightarrow \dot{\bigcup}_{z \in \Omega} \mathcal{O}_{\mathscr{B}, z} \# D_{r}\left(U_{z}^{(e)}\right)$ satisfying (1). It will be convenient to call an open covering $\Omega \cap \operatorname{St}(F)=\bigcup_{i \in I} \Omega_{i}$ together with the elements $s_{i}$ such that (a) and (b) hold a datum for $s$ with respect to the facet $F$. Any open covering of $\Omega \cap \operatorname{St}(F)$ which is a refinement of the covering $\left\{\Omega_{i}\right\}_{i \in I}$, together with the same set of elements $s_{i}$ is again a datum for $s$ with respect to $F$.

Suppose $\Omega^{\prime} \subseteq \Omega$ is an open subset and let $s \in\left(\mathcal{O}_{\mathscr{B}} \# \underline{D}_{r}\right)(\Omega)$. Let $F \subseteq \mathscr{B}$ be a facet. Given a corresponding datum $\left\{\Omega_{i}\right\}_{i \in I}$ for $s$ put $\Omega_{i}^{\prime}:=\Omega^{\prime} \cap \Omega_{i}$. Together with the elements $s_{i}$, in case $\Omega_{i}^{\prime} \cap F \neq \varnothing$, we obtain a datum for the function $\left.s\right|_{\Omega^{\prime}}$. It follows that $\left(\mathcal{O}_{\mathscr{B}} \# \underline{D}_{r}\right)$ is a presheaf of $K$-vector spaces on $\mathscr{B}$.

In the following it will be convenient to define $\mathcal{F}(\Omega)$ as the $K$-vector space of all maps

$$
s: \Omega \rightarrow \bigcup_{z \in \Omega} \mathcal{O}_{\mathscr{B}, z} \#_{L} D_{r}\left(U_{z}^{(e)}\right)
$$

satisfying condition (1) in Definition 7.2.1. It is clear that pointwise multiplication makes $\mathcal{F}$ a sheaf of $K$-algebras on $\mathscr{B}$ such that $\left(\mathcal{O}_{\mathscr{B}} \# \underline{D}_{r}\right)$ is a subpresheaf of $K$-vector spaces.

Lemma 7.2.2. The induced multiplication makes $\left(\mathcal{O}_{\mathscr{B}} \# \underline{D}_{r}\right) \subseteq \mathcal{F}$ an inclusion of sheaves of $K$-algebras.

Proof. Take an open subset $\Omega \subseteq \mathscr{B}$. We first show that for $s, s^{\prime} \in\left(\mathcal{O}_{\mathscr{B}} \# \underline{D}_{r}\right)(\Omega)$ we have $s s^{\prime} \in\left(\mathcal{O}_{\mathscr{B}} \# \underline{D}_{r}\right)(\Omega)$, i.e., that $\left(\mathcal{O}_{\mathscr{B}} \# \underline{D}_{r}\right)(\Omega)$ is a subalgebra of $\mathcal{F}(\Omega)$.

To do this let $F \subseteq \mathscr{B}$ be a facet. Let $\left\{\Omega_{i}\right\}_{i \in I}$ and $\left\{\Omega_{j}^{\prime}\right\}_{j \in J}$ be corresponding data for $s$ and $s^{\prime}$ respectively. Passing to $\left\{\Omega_{i j}\right\}_{i j}$ with $\Omega_{i j}=\Omega_{i} \cap \Omega_{j}^{\prime}$ and refining the coverings if necessary, we may assume: there exists one datum $\left\{\Omega_{i}\right\}_{i \in I}$ for both $s, s^{\prime}$ and each $\Omega_{i}$ is contained in a compact subset of $\operatorname{St}(F)$. We will produce a datum for $s s^{\prime}$ by passing to a suitable open covering of $\Omega_{i}$ whenever $\Omega_{i}$ and $F$ intersect. To this end, let us fix such an $i \in I$. We choose connected compact subsets $C \subset \operatorname{St}(F)$ whose open interiors $C^{\circ}$ form a covering of $\Omega_{i}$. We have the $K$-algebra $\mathcal{O}_{\mathscr{B}}(C) \# D_{r}\left(U_{F}^{(e)}\right)$ from Proposition 6.4.15. We apply the base change $(\cdot) \hat{\otimes}_{L} D_{r}\left(U_{F}^{(e)}\right)$ to the restriction map $\mathcal{O}_{\mathscr{B}}(\Omega) \rightarrow \mathcal{O}_{\mathscr{B}}(C)$ and consider the image of $s_{i}$ and $s_{i}^{\prime}$ in $\mathcal{O}_{\mathscr{B}}(C) \# D_{r}\left(U_{F}^{(e)}\right)$. Let $s_{i} s_{i}^{\prime} \in \mathcal{O}_{\mathscr{B}}(C) \# D_{r}\left(U_{F}^{(e)}\right)$ be their product. We apply the base change $(\cdot) \hat{\otimes}_{L} D_{r}\left(U_{F}^{(e)}\right)$ to the restriction map $\mathcal{O}_{\mathscr{B}}(C) \rightarrow \mathcal{O}_{\mathscr{B}}\left(C^{\circ}\right)$ and consider the image of $s_{i} s_{i}^{\prime}$ in $\mathcal{O}_{\mathscr{B}}\left(C^{\circ}\right) \hat{\otimes}_{L} D_{r}\left(U_{F}^{(e)}\right)$. We denote this image again by $s_{i} s_{i}^{\prime}$. According to the definition of the product on $\mathcal{O}_{\mathscr{B}}(C) \# D_{r}\left(U_{F}^{(e)}\right)$ in Proposition 6.4.15 we find, for any $z \in C^{\circ} \cap F$, that

$$
\left(s s^{\prime}\right)(z)=s(z) s^{\prime}(z)=\left(\iota_{z} \hat{\otimes} \mathrm{id}\right)\left(s_{i}\right) \cdot\left(\iota_{z} \hat{\otimes} \mathrm{id}\right)\left(s_{i}^{\prime}\right)=\left(\iota_{z} \hat{\otimes} \mathrm{id}\right)\left(s_{i} s_{i}^{\prime}\right)
$$

using Corollary 6.4.10 and we find, for any $z^{\prime} \in C^{\circ}$, that

$$
\left(s s^{\prime}\right)\left(z^{\prime}\right)=s\left(z^{\prime}\right) s^{\prime}\left(z^{\prime}\right)=\left(\iota_{z} \hat{\otimes} \sigma_{r}^{F^{\prime} F}\right)\left(s_{i}\right) \cdot\left(\iota_{z} \hat{\otimes} \sigma_{r}^{F^{\prime} F}\right)\left(s_{i}^{\prime}\right)=\left(\iota_{z} \hat{\otimes} \sigma_{r}^{F^{\prime} F}\right)\left(s_{i} s_{i}^{\prime}\right)
$$


using Lemma 6.4.13 ( $F^{\prime}$ is the unique facet in $\operatorname{St}(F)$ that contains $\left.z^{\prime}\right)$. This shows that, if we replace each such $\Omega_{i}$ by the open covering given by the corresponding $C^{\circ}$ and invoke the corresponding sections $s_{i} s_{i}^{\prime} \in \mathcal{O}_{\mathscr{B}}\left(C^{\circ}\right) \hat{\otimes}_{L} D_{r}\left(U_{F}^{(e)}\right)$, we will have a datum for $s s^{\prime}$ relative to $F$. Consequently, $s s^{\prime} \in\left(\mathcal{O}_{\mathscr{B}} \# \underline{D}_{r}\right)(\Omega)$ and hence, $\left(\mathcal{O}_{\mathscr{B}} \# \underline{D}_{r}\right)(\Omega)$ is a subalgebra of $\mathcal{F}(\Omega)$. If $\Omega^{\prime} \subseteq \Omega$ is an open subset the restriction map $\left(\mathcal{O}_{\mathscr{B}} \# \underline{D}_{r}\right)(\Omega) \rightarrow\left(\mathcal{O}_{\mathscr{B}} \# \underline{D}_{r}\right)\left(\Omega^{\prime}\right)$ is obviously multiplicative. Thus, $\left(\mathcal{O}_{\mathscr{B}} \# \underline{D}_{r}\right)$ is a presheaf of $K$-algebras.

Let us show that $\left(\mathcal{O}_{\mathscr{B}} \# \underline{D}_{r}\right)$ is in fact a sheaf. Since $\left(\mathcal{O}_{\mathscr{B}} \# \underline{D}_{r}\right) \subseteq \mathcal{F}$ is a subpresheaf and $\mathcal{F}$ is a sheaf it suffices to prove the following: if

$$
\Omega=\bigcup_{j \in J} U_{j}
$$

is an open covering of an open subset $\Omega \subseteq \mathscr{B}$ and if $s_{j} \in\left(\mathcal{O}_{\mathscr{B}}^{\#} \underline{D}_{r}\right)\left(U_{j}\right)$ are local sections with $\left.s_{j}\right|_{U_{j} \cap U_{i}}=\left.s_{i}\right|_{U_{i} \cap U_{j}}$ for all $i, j \in J$ then the unique section $s \in \mathcal{F}(\Omega)$ with $\left.s\right|_{U_{j}}=s_{j}$ for all $j \in J$ lies in $\left(\mathcal{O}_{\mathscr{B}} \# \underline{D}_{r}\right)(\Omega)$. To do this let $F \subseteq \mathscr{B}$ be a facet. Consider for each $j \in J$ a datum $\left\{U_{j i}\right\}_{i \in I}$ for $s_{j}$. In particular, $U_{j} \cap \operatorname{St}(F)=\bigcup_{i \in I} U_{j i}$ and there are distinguished elements

$$
s_{j i} \in \mathcal{O}_{\mathscr{B}}\left(U_{j i}\right) \hat{\otimes}_{L} D_{r}\left(U_{F}^{(e)}\right)
$$

whenever $U_{j i} \cap F \neq \varnothing$ intersect. Then $\Omega \cap \operatorname{St}(F)=\bigcup_{j i} U_{j i}$ (together with the elements $s_{j i}$ whenever $U_{j i} \cap F \neq \varnothing$ ) is a datum for $s$. Indeed, given $z \in U_{j i} \cap F$ one has $s(z)=s_{j}(z)=\left(\iota_{z} \hat{\otimes} \mathrm{id}\right)\left(s_{j i}\right)$ which shows condition (a) in Definition 7.2.1. Moreover, if $z^{\prime} \in U_{j i}$ one has $s\left(z^{\prime}\right)=s_{j}\left(z^{\prime}\right)=\left(\iota_{z} \hat{\otimes} \sigma_{r}^{F^{\prime} F}\right)\left(s_{j i}\right)$, which shows (b). Together this means $s \in\left(\mathcal{O}_{\mathscr{B}} \# \underline{D}_{r}\right)(\Omega)$.

The next lemma shows that the stalks of the sheaf $\left(\mathcal{O}_{\mathscr{B}} \# \underline{D}_{r}\right)$ are as expected.

Lemma 7.2.3. The canonical map $\left(\mathcal{O}_{\mathscr{B}} \# \underline{D}_{r}\right)_{z} \stackrel{\sim}{\rightarrow} \mathcal{O}_{\mathscr{B}, z} \# D_{r}\left(U_{z}^{(e)}\right)$ is an isomorphism of $K$-algebras for any $z \in \mathscr{B}$.

Proof. There is the $K$-algebra homomorphism

$$
\left(\mathcal{O}_{\mathscr{B}} \# \underline{D}_{r}\right)_{z} \rightarrow \mathcal{O}_{\mathscr{B}, z} \# D_{r}\left(U_{z}^{(e)}\right), \quad \text { germ of } s \text { at } z \mapsto s(z) .
$$

Let us show that this map is injective. Let $[s]$ be the germ of a local section $s \in\left(\mathcal{O}_{\mathscr{B}} \# \underline{D}_{r}\right)(\Omega)$ over some open subset $\Omega \subseteq \mathscr{B}$ with the property $s(z)=0$. Let $F$ be the unique facet of $\mathscr{B}$ that contains $z$ and let $\left\{\Omega_{i}\right\}_{i \in I}$ be a corresponding datum for $s$. According to Lemma 6.2.4 we may write the stalk

$$
\mathcal{O}_{\mathscr{B}, z}=\underset{\mathrm{l}}{\lim _{\mathrm{W}}} \mathcal{A}_{V}
$$

as a compact inductive limit of integral affinoid algebras with injective transition maps. 
Let us abbreviate $\mathcal{E}:=D_{r}\left(U_{F}^{(e)}\right)$. If $W \subseteq V$ is an inclusion of affinoids occurring in the above inductive limit, then [Emerton 2011, Corollary 1.1.27] implies that the base changed map

$$
\mathcal{A}_{V} \hat{\otimes}_{L} \mathcal{E} \rightarrow \mathcal{A}_{W} \hat{\otimes}_{L} \mathcal{E}
$$

remains injective. Let $i_{0} \in I$ such that $z \in \Omega_{i_{0}} \cap F$ and consider the map

$$
\iota_{z} \hat{\otimes} \mathrm{id}: \mathcal{O}_{\mathscr{B}}\left(\Omega_{i_{0}}\right) \hat{\otimes}_{L} \mathcal{E} \rightarrow \mathcal{O}_{\mathscr{B}, z} \hat{\otimes}_{L} \mathcal{E} \simeq \underset{V}{\lim }\left(\mathcal{A}_{V} \hat{\otimes}_{L} \mathcal{E}\right)
$$

The last isomorphism here is due to Proposition 2.4.1. Let $V$ be an affinoid in the inductive limit on the right-hand side such that $\mathcal{A}_{V} \hat{\otimes}_{L} \mathcal{E}$ contains the image of $s_{i_{0}}$ under $\iota_{z} \hat{\otimes}$ id. Choose an open subset $U \subseteq X^{\text {an }}$ in $V$ containing $z$ and replace $\Omega_{i_{0}}$ by the intersection $\mathscr{B} \cap U$. Then replace $s_{i_{0}}$ by its restriction to this intersection, in other words, $s_{i_{0}}$ lies now in the image of the map

$$
\mathcal{A}_{V} \hat{\otimes}_{L} \mathcal{E} \rightarrow \mathcal{O}_{\mathscr{B}}\left(\Omega_{i_{0}}\right) \hat{\otimes}_{L} \mathcal{E} .
$$

By our discussion above, the natural map from $\mathcal{A}_{V} \hat{\otimes}_{L} \mathcal{E}$ into $\mathcal{O}_{\mathscr{B}, z} \hat{\otimes}_{L} \mathcal{E}$ is injective and lifts the map $\iota_{z} \hat{\otimes}$ id. We may therefore deduce from

$$
0=s(z)=\left(\iota_{z} \hat{\otimes} \mathrm{id}\right)\left(s_{i_{0}}\right)
$$

that $s_{i_{0}}=0$. Given $z^{\prime} \in \Omega_{i_{0}}$ let $F^{\prime}$ be the unique facet of $\operatorname{St}(F)$ containing $z^{\prime}$. Then $s\left(z^{\prime}\right)=\left(\iota_{z} \hat{\otimes} \sigma_{r}^{F^{\prime} F}\right)\left(s_{i_{0}}\right)=0$ according to condition (b) and, consequently, $\left.s\right|_{\Omega_{i_{0}}}=0$. Since $\Omega_{i_{0}}$ is an open neighborhood of $z$ this shows $[s]=0$ and proves injectivity.

Let us now show that our map is surjective. Let $t \in \mathcal{O}_{\mathscr{B}, z} \# D_{r}\left(U_{z}^{(e)}\right)$ be an element in the target. Since the stalk $\mathcal{O}_{\mathscr{B}, z}$ is an inductive limit with compact and injective transition maps and since $D_{r}\left(U_{z}^{(e)}\right)$ is a Banach space, Proposition 2.4.1 implies that there is an open neighborhood $\Omega^{\prime}$ of $z$ and an element $\tilde{s} \in \mathcal{O}_{\mathscr{B}}\left(\Omega^{\prime}\right) \hat{\otimes}_{L} D_{r}\left(U_{z}^{(e)}\right)$ such that $\left(\iota_{z} \hat{\otimes} \mathrm{id}\right)(\tilde{s})=t$. Let $F \subseteq \mathscr{B}$ be a facet containing $z$ and define

$$
\Omega:=\Omega^{\prime} \cap \operatorname{St}(F), \quad s:=\left(\operatorname{res}_{\Omega}^{W} \hat{\otimes} \mathrm{id}\right)(\tilde{s}) \in \mathcal{O}_{\mathscr{B}}(\Omega) \hat{\otimes}_{L} D_{r}\left(U_{z}^{(e)}\right) .
$$

Since $\operatorname{St}(F)$ is an open neighborhood of $z$ and contains only finitely many facets of $\mathscr{B}$ we may pass to a smaller $\Omega^{\prime}$ (and hence $\Omega$ ) and therefore assume: any $F^{\prime} \in(\mathscr{B} \backslash \operatorname{St}(F))$ satisfies $F^{\prime} \cap \Omega=\varnothing$. For any $z^{\prime} \in \Omega$ let $s\left(z^{\prime}\right):=\left(\iota_{z^{\prime}} \hat{\otimes} \sigma_{r}^{F F^{\prime}}\right)(s)$ where $F^{\prime}$ denotes the facet in $\operatorname{St}(F)$ containing $z^{\prime}$. This defines a function

$$
s: \Omega \rightarrow \bigcup_{z^{\prime} \in \Omega}^{\cdot} \mathcal{O}_{\mathscr{B}, z} \# D_{r}\left(U_{z^{\prime}}^{(e)}\right)
$$

satisfying condition (1) of Definition 7.2.1. According to Lemma 4.3.5 one has $\sigma_{r}^{F F}=\mathrm{id}$ whence

$$
s(z)=\left(\iota_{z} \hat{\otimes} \mathrm{id}\right)(s)=\left(\iota_{z} \hat{\otimes} \mathrm{id}\right)(\tilde{s})=t .
$$


Thus, the germ of $s$ at $z$ will be a preimage of $t$ once we have shown that $s \in\left(\mathcal{O}_{\mathscr{B}} \# \underline{D}_{r}\right)(\Omega)$. To do this consider an arbitrary facet $F^{\prime} \subset \mathscr{B}$ together with the covering of $\Omega \cap \operatorname{St}\left(F^{\prime}\right)$ consisting of the single element

$$
\Omega_{0}:=\Omega \cap \operatorname{St}\left(F^{\prime}\right) .
$$

Suppose $\Omega_{0} \cap F^{\prime} \neq \varnothing$. We have to exhibit an element $s_{0} \in \mathcal{O}_{\mathscr{B}}\left(\Omega_{0}\right) \hat{\otimes}_{L} D_{r}\left(U_{F^{\prime}}^{(e)}\right)$ satisfying conditions (a) and (b) in Definition 7.2.1. Since $F^{\prime} \in \operatorname{St}(F)$ we may define $s_{0}:=\left(\operatorname{id} \hat{\otimes} \sigma_{r}^{F F^{\prime}}\right)(s)$. For any $z^{\prime} \in \Omega \cap F^{\prime}$ we compute

$$
s\left(z^{\prime}\right)=\left(\iota_{z^{\prime}} \hat{\otimes} \sigma_{r}^{F F^{\prime}}\right)(s)=\left(\iota_{z^{\prime}} \hat{\otimes} \mathrm{id}\right)\left(\mathrm{id} \hat{\otimes} \sigma_{r}^{F F^{\prime}}\right)(s)=\left(\iota_{z^{\prime}} \hat{\otimes} \mathrm{id}\right)\left(s_{0}\right)
$$

which shows (a). Moreover, for any $z^{\prime} \in \Omega_{0}$ we compute

$$
s\left(z^{\prime}\right)=\left(\iota_{z^{\prime}} \hat{\otimes} \sigma_{r}^{F F^{\prime \prime}}\right)(s)=\left(\iota_{z^{\prime}} \hat{\otimes} \sigma_{r}^{F^{\prime} F^{\prime \prime}}\right)\left(\iota_{z^{\prime}} \hat{\otimes} \sigma_{r}^{F F^{\prime}}\right)(s)=\left(\iota_{z^{\prime}} \hat{\otimes} \sigma_{r}^{F^{\prime} F^{\prime \prime}}\right)\left(s_{0}\right)
$$

by Lemma 4.3.5. Here $F^{\prime \prime}$ denote the facet of $\operatorname{St}\left(F^{\prime}\right)$ that contains $z^{\prime}$. This shows (b) and completes the proof.

Corollary 7.2.4. The $\mathcal{O}_{\mathscr{B}, z}$-module structure on $\left(\mathcal{O}_{\mathscr{B}} \# \underline{D}_{r}\right)_{z}$ for any $z \in \mathscr{B}$ sheafifies to a $\mathcal{O}_{\mathscr{B}}$-module structure on $\left(\mathcal{O}_{\mathscr{B}} \# \underline{D}_{r}\right)$ (compatible with scalar multiplication by $L)$.

Proof. As with any sheaf [Godement 1958, II.1.2] we may regard $\left(\mathcal{O}_{\mathscr{B}} \# \underline{D}_{r}\right)$ as the sheaf of continuous sections of its étale space

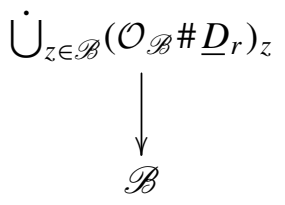

and the same applies to the sheaf $\mathcal{O}_{\mathscr{B}}$. By the preceding proposition we have $\left(\mathcal{O}_{\mathscr{B}} \# \underline{D}_{r}\right)_{z}=\mathcal{O}_{\mathscr{B}, z} \# D_{r}\left(U_{z}^{(e)}\right)$ for any $z \in \mathscr{B}$. Let $\Omega \subseteq \mathscr{B}$ be an open subset, $s \in\left(\mathcal{O}_{\mathscr{B}} \# \underline{D}_{r}\right), f \in \mathcal{O}_{\mathscr{B}}(\Omega)$. For $z \in \Omega$ put $(f \cdot s)(z):=f(z) \cdot s(z)$. This visibly defines an element $f \cdot s \in \mathcal{F}(\Omega)$. The " $\mathcal{O}_{\mathscr{B}}$-linearity" in conditions (a) and (b) proves $f \cdot s \in\left(\mathcal{O}_{\mathscr{B}} \# \underline{D}_{r}\right)$. It follows that $\left(\mathcal{O}_{\mathscr{B}} \# \underline{D}_{r}\right)$ is an $\mathcal{O}_{\mathscr{B}}$-module in the prescribed way.

\section{Proposition 7.2.5. The natural map}

$$
D_{r}\left(U_{z}^{(e)}\right) \rightarrow\left(\mathcal{O}_{\mathscr{B}} \# \underline{D}_{r}\right)_{z}, \quad \delta \mapsto 1 \hat{\otimes} \delta,
$$

sheafifies to a morphism of sheaves of $K$-algebras $\underline{D}_{r} \rightarrow \mathcal{O}_{\mathscr{B}} \# \underline{D}_{r}$.

Proof. This is easy to see. 
Recall from (6.4.12) that we have for any $z \in \mathscr{B}$ a canonical $K$-algebra homomorphism

$$
\mathcal{O}_{\mathscr{B}, z} \# U(\mathfrak{g})_{K} \rightarrow \mathcal{O}_{\mathscr{B}, z} \# D_{r}\left(U_{z}^{(e)}\right) .
$$

Proposition 7.2.6. The homomorphisms (6.4.12) sheafify into a morphism

$$
\mathcal{O}_{\mathscr{B}} \# U(\mathfrak{g})_{K} \rightarrow \mathcal{O}_{\mathscr{B}} \# \underline{D}_{r}
$$

of sheaves of $K$-algebras. This morphism is $\mathcal{O}_{\mathscr{B}}$-linear.

Proof. We view $\mathcal{O}_{\mathscr{B}} \# U(\mathfrak{g})_{K}$ as the sheaf of continuous sections of its étale space

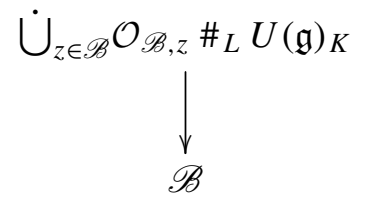

Composing such a section with (6.4.12) defines a morphism $i: \mathcal{O}_{\mathscr{B}} \# U(\mathfrak{g})_{K} \rightarrow \mathcal{F}$ of sheaves of $K$-algebras and we will prove that its image lies in the subsheaf $\left(\mathcal{O}_{\mathscr{B}} \# \underline{D}_{r}\right)$. To do this let $\Omega \subseteq \mathscr{B}$ be an open subset and $s \in \mathcal{O}_{\mathscr{B}} \# U(\mathfrak{g})_{K}(\Omega)$ a local section.

Let $F \subseteq \mathscr{B}$ be a facet. Consider the covering of $\Omega \cap \operatorname{St}(F)$ consisting of the single element $\Omega_{0}:=\Omega \cap \operatorname{St}(F)$. In case $\Omega_{0} \cap F \neq \varnothing$ let $s_{0}$ be the image of $\tilde{s}$ under the map

$$
\mathcal{O}_{\mathscr{B}}(\Omega) \otimes_{L} U(\mathfrak{g})_{K} \rightarrow \mathcal{O}_{\mathscr{B}}(\Omega) \hat{\otimes}_{L} D_{r}\left(U_{F}^{(e)}\right)
$$

induced by $U(\mathfrak{g})_{K} \subseteq D_{r}\left(U_{F}^{(e)}\right)$. For any $z \in \Omega_{0} \cap F$ we obviously have $i(s)(z)=$ $\left(\iota_{z} \hat{\otimes} \mathrm{id}\right)\left(s_{0}\right)$, which shows condition (a). For any $z \in \Omega_{0}$ we find $i(s)(z)=\left(\iota_{z} \hat{\otimes}\right.$ id) $\left(s_{0}\right)=\left(\iota_{z} \hat{\otimes} \sigma_{r}^{F F^{\prime}}\right)\left(s_{0}\right)$ by the last statement of Lemma 4.3.5. Here $F^{\prime}$ denotes the facet containing $z$. This shows (b). In the light of the definitions it is clear that the resulting morphism $\mathcal{O}_{\mathscr{B}} \# U(\mathfrak{g})_{K} \rightarrow \mathcal{O}_{\mathscr{B}} \# \underline{D}_{r}$ is $\mathcal{O}_{\mathscr{B}}$-linear.

7.3. Infinitesimal characters. We will write $\mathfrak{g}_{K}:=\mathfrak{g} \otimes_{\mathbb{Q}_{p}} K, \mathfrak{t}_{K}:=\mathfrak{t} \otimes_{\mathbb{Q}_{p}} K$ etc.

7.3.1. According to [Schneider and Teitelbaum 2002, Proposition 3.7] the ring $Z\left(\mathfrak{g}_{K}\right)$ lies in the center of the ring $D(G)$. In the following we fix a central character

$$
\theta: Z\left(\mathfrak{g}_{K}\right) \rightarrow K
$$

and we let

$$
D(G)_{\theta}:=D(G) \otimes Z\left(\mathfrak{g}_{K}\right), \theta
$$

be the corresponding central reduction of $D(G)$. A (left) $D(G)_{\theta}$-module $M$ is called coadmissible if it is coadmissible as $D(G)$-module via the natural map $D(G) \rightarrow D(G)_{\theta}, \delta \mapsto \delta \hat{\otimes} 1$. In the following we will study the abelian category of coadmissible $D(G)_{\theta}$-modules. As explained in the beginning, this category is 
antiequivalent to the category of admissible locally analytic $G$-representations over $K$ which have infinitesimal character $\theta$.

Example. Let $\lambda_{0}: D(T) \rightarrow K$ denote the character of $D(T)$ induced by the augmentation map $K[T] \rightarrow K$. The restriction of $\lambda_{0}$ to the Lie algebra $\mathfrak{t}_{K} \subset D(T)$ vanishes identically whence $\chi=\rho$. Let $\theta_{0}: Z\left(\mathfrak{g}_{K}\right) \rightarrow K$ be the infinitesimal character associated to $\rho$ via the Harish-Chandra homomorphism. Then $\operatorname{ker} \theta_{0}=$ $Z\left(\mathfrak{g}_{K}\right) \cap U\left(\mathfrak{g}_{K}\right) \mathfrak{g}_{K}$.

Remark. K. Ardakov and S. Wadsley [2013, Section 8] have established a version of Quillen's lemma for $p$-adically completed universal enveloping algebras. It implies that any topologically irreducible admissible locally analytic $G$-representation admits, up to a finite extension of $K$, a central character and an infinitesimal character [Dospinescu and Schraen 2013].

7.3.2. To investigate the local situation let $F$ be a facet in $\mathscr{B}$. We have

$$
Z\left(\mathfrak{g}_{K}\right) \subseteq D\left(U_{F}^{(e)}\right) \cap Z(D(G)) \subseteq Z\left(D\left(U_{F}^{(e)}\right)\right),
$$

again according to [Schneider and Teitelbaum 2002, Proposition 3.7]. We let

$$
D_{r}\left(U_{F}^{(e)}\right)_{\theta}:=D_{r}\left(U_{F}^{(e)}\right) \otimes_{Z(\mathfrak{g})_{K}, \theta} K
$$

be the corresponding central reduction of $D_{r}\left(U_{F}^{(e)}\right)$.

Let $F, F^{\prime}$ be two facets in $\mathscr{B}$ such that $F^{\prime} \subseteq \bar{F}$ and consider the homomorphism $\sigma_{r}^{F^{\prime} F}$. According to the last statement of Lemma 4.3.5 it factors by continuity into a homomorphism

$$
\sigma_{r}^{F^{\prime} F}: D_{r}\left(U_{F^{\prime}}^{(e)}\right)_{\theta} \rightarrow D_{r}\left(U_{F}^{(e)}\right)_{\theta}
$$

We may therefore define a sheaf of $K$-algebras $\underline{D}_{r, \theta}$ in complete analogy with the sheaf $\underline{D}_{r}$ by replacing each $D_{r}\left(U_{z}^{(e)}\right)$ and each $D_{r}\left(U_{F}^{(e)}\right)$ by their central reductions. In particular, $\left(\underline{D}_{r, \theta}\right)_{z}=D_{r}\left(U_{z}^{(e)}\right)_{\theta}$ for any $z \in \mathscr{B}$ and there is an obvious quotient morphism

$$
\underline{D}_{r} \rightarrow \underline{D}_{r, \theta}
$$

7.4. Twisting. We now bring in a toral character

$$
\chi: \mathfrak{t}_{K} \rightarrow K
$$

such that $\sigma(\chi)=\theta$. We consider the two-sided ideals $\mathcal{I}_{\mathscr{B}, \mathrm{t}}^{\text {an }}$ and $\mathcal{I}_{\mathscr{B}, \chi}^{\text {an }}$ of $\mathcal{O}_{\mathscr{B}} \# U\left(\mathfrak{g}_{K}\right)$. Denote the right ideal in $\mathcal{O}_{\mathscr{B}} \# \underline{D_{r}}$ generated by the image of the first resp. second under the morphism

$$
\mathcal{O}_{\mathscr{B}} \# U\left(\mathfrak{g}_{K}\right) \rightarrow \mathcal{O}_{\mathscr{B}} \# \underline{D}_{r}
$$

by $\mathscr{I}_{t}^{\text {an }}$ resp. $\mathscr{I}_{\chi}^{\text {an }}$.

Recall that we assume $e>e_{\text {st }}$ throughout this section and that this implies $e>e_{\text {uni }}$. 
Proposition 7.4.1. Let $z \in \mathscr{B}$ a point and let $V$ be a strictly affinoid neighborhood of $z$ on which $U_{z}^{(e-1)}$ acts analytically. Then the ring $\mathcal{A}_{V} \# D_{r_{0}}\left(U_{z}^{(e)}\right)$ is noetherian. Proof. As $e>e_{\text {uni }}$ the group $U_{z}^{(e-1)}$ is a uniform pro-p group. Let

$$
\mathfrak{h}_{\mathbb{Z}_{p}}=\mathcal{L}\left(U_{z}^{(e-1)}\right) \subset \mathfrak{g}
$$

be the $\mathbb{Z}_{p}$-Lie algebra of $U_{z}^{(e-1)}$ [Dixon et al. 1999, Section 9.4]. We consider the bijective exponential map exp $: \mathfrak{h}_{\mathbb{Z}_{p}} \rightarrow U_{z}^{(e-1)}$ which is used to define the affinoid analytic subgroup $\mathbb{U}_{z}^{(e-1)} \subset G^{\text {an }}$; see Section 6.2.5. This exponential map gives then rise to an exponential map of affinoid analytic spaces exp : $\mathbb{B} \otimes_{\mathbb{Z}_{p}} \mathfrak{h}_{\mathbb{Z}_{p}} \rightarrow \mathbb{U}_{z}^{(e-1)}$, where $\mathbb{B}$ is the closed unit disc over $\mathbb{Q}_{p}$ and $\mathbb{B} \otimes_{\mathbb{Z}_{p}} \mathfrak{h}_{\mathbb{Z}_{p}}$ is the strictly $\mathbb{Q}_{p}$-analytic space whose affinoid algebra is

$$
\operatorname{Sym}_{\mathbb{Z}_{p}}\left(\mathfrak{h}_{\mathbb{Z}_{p}}^{\vee}\right)^{\wedge} \otimes_{\mathbb{Z}_{p}} \mathbb{Q}_{p} .
$$

Here, $\mathfrak{h}_{\mathbb{Z}_{p}}^{\vee}=\operatorname{Hom}_{\mathbb{Z}_{p}}\left(\mathfrak{h}_{\mathbb{Z}_{p}}, \mathbb{Z}_{p}\right)$ and $(\cdot)^{\wedge}$ means the $p$-adic completion.

The affinoid algebra $\mathcal{A}_{V}$ is a $\mathfrak{g}$-module. As a first step we want to show that the subring $A \subset \mathcal{A}_{V}$ of power-bounded elements is stable under the action of $\mathfrak{h}_{\mathbb{Z}_{p}}$. Because $U_{z}^{(e-1)}$ acts analytically on $V$ we have for any $\mathfrak{x} \in \mathfrak{h}_{\mathbb{Z}_{p}}$ and $f \in A$

$$
\exp (t \mathfrak{x}) \cdot f=\sum_{n \geq 0}\left(\frac{\mathfrak{x}^{n}}{n !} \cdot f\right) t^{n},
$$

where the right-hand side is a convergent power series in $t \in \mathbb{B}$. If we evaluate this identity at a point $z^{\prime} \in V$ we get

$$
f\left(\exp (-t \mathfrak{x}) \cdot z^{\prime}\right)=\sum_{n}\left(\frac{\mathfrak{x}^{n}}{n !} \cdot f\right)\left(z^{\prime}\right) t^{n},
$$

which holds for all $t \in \mathbb{B}$. The left-hand side of (7.4.2) is bounded by 1 in absolute value for all $t \in \mathbb{B}$, and so is the right-hand side. But this means that all coefficients $\left(\frac{1}{n !} \mathfrak{x}^{n} . f\right)\left(z^{\prime}\right) \in \mathcal{A}_{V}$ on the right-hand side of (7.4.2) must be bounded by one in absolute value, and, in particular, the coefficient $(\mathfrak{x} . f)\left(z^{\prime}\right)$. This shows that the supremum norm of $\mathfrak{x} . f$ on $V$ is bounded by 1, i.e., that we have $\mathfrak{x} . f \in A$.

We let $U\left(\mathfrak{h}_{\mathbb{Z}_{p}}\right)$ be the universal enveloping algebra over $\mathbb{Z}_{p}$ of $\mathfrak{h}_{\mathbb{Z}_{p}}$. As we have seen above, the ring $A$ is a $U\left(\mathfrak{h}_{\mathbb{Z}_{p}}\right)$-module, and we can consider the skew enveloping algebra $A \# U\left(\mathfrak{h}_{\mathbb{Z}_{p}}\right):=A \otimes_{\mathbb{Z}_{p}} U\left(\mathfrak{h}_{\mathbb{Z}_{p}}\right)$. We denote its $p$-adic completion by

$$
R_{A}:=A \# \hat{U}\left(\mathfrak{h}_{\mathbb{Z}_{p}}\right)
$$

In a manner completely analogous to Section 3 , this becomes a $p$-adically complete topological $\mathbb{Z}_{p}$-algebra. Its $\bmod p$-reduction is equal to

$$
g r_{0}\left(R_{A}\right):=\bar{A} \# U\left(\mathfrak{h}_{\mathbb{F}_{p}}\right)
$$


where $\bar{A}=A / p A$ and $\mathfrak{h}_{\mathbb{F}_{p}}:=\mathfrak{h}_{\mathbb{Z}_{p}} \otimes \mathbb{F}_{p}$. The vector space underlying $g r_{0}\left(R_{A}\right)$ equals $\bar{A} \otimes_{\mathbb{F}_{p}} U\left(\mathfrak{h}_{\mathbb{F}_{p}}\right)$. The second factor in this tensor product has its PBW-filtration. It induces a positive $\mathbb{Z}$-filtration on $g r_{0}\left(R_{A}\right)$ with $\bar{A}$ concentrated in degree zero. Let deg be the degree function of this filtration. If $f \in \bar{A}, \mathfrak{x} \in \mathfrak{h}_{\mathbb{F}_{p}}$ we have $[f, \mathfrak{x}]=\mathfrak{x}(f)$ from which it follows that $g r_{0}\left(R_{A}\right)$ is a $\mathbb{Z}$-filtered ring. Moreover,

$$
\operatorname{deg}[f, \mathfrak{x}]<\operatorname{deg} \mathfrak{x}
$$

which means that the associated graded ring

$$
\operatorname{Gr}\left(R_{A}\right):=\operatorname{gr} g r_{0}\left(R_{A}\right)=\bar{A} \otimes_{\mathbb{F}_{p}} S\left(\mathfrak{h}_{\mathbb{F}_{p}}\right)
$$

is commutative and therefore a polynomial ring over $\bar{A}$. Since $\bar{A}$ is noetherian, so is $\operatorname{Gr}\left(R_{A}\right)$. By [Schneider and Teitelbaum 2003, Proposition 1.1] the ring $g r_{0}\left(R_{A}\right)$ is noetherian. Now $R_{A}$ is complete with respect to the $p$-adic topology and the graded ring associated with the $p$-adic filtration equals

$$
\operatorname{gr}\left(R_{A}\right)=\left(g r_{0} R_{A}\right)\left[Z, Z^{-1}\right],
$$

the Laurent polynomials over $g r_{0}\left(R_{A}\right)$ in one variable $Z$ (e.g., [Ardakov and Wadsley 2013, Lemma 3.1]). It is noetherian, since $g r_{0}\left(R_{A}\right)$ is noetherian. Another application of [Schneider and Teitelbaum 2003, Proposition 1.2] now yields that $R_{A}$ is noetherian. The embedding $\mathfrak{h}_{\mathbb{Z}_{p}} \subset \mathfrak{g} \subset D\left(U_{z}^{(e)}\right)$ induces a ring isomorphism

$$
\mathbb{Q}_{p} \otimes_{\mathbb{Z}_{p}} \hat{U}\left(\mathfrak{h}_{\mathbb{Z}_{p}}\right) \stackrel{\sim}{\longrightarrow} D_{r_{0}}\left(U_{z}^{(e)}, \mathbb{Q}_{p}\right) ;
$$

see [Schmidt 2013, Proposition 6.3]. We have $\mathbb{Q}_{p} \otimes_{\mathbb{Z}_{p}} A=\mathcal{A}_{V}$ and thus a ring isomorphism

$$
\mathbb{Q}_{p} \otimes_{\mathbb{Z}_{p}} R_{A} \simeq \mathcal{A}_{V} \# D_{r_{0}}\left(U_{z}^{(e)}, \mathbb{Q}_{p}\right)
$$

Therefore, the right-hand side is noetherian. Base change from $\mathbb{Q}_{p}$ to $K$ finally yields the assertion of the lemma.

Lemma 7.4.3. For all $m \geq 0$ the inclusion $U_{z}^{(e+m)} \subseteq U_{z}^{(e)}$ induces a finite free ring homomorphism

$$
D_{r_{0}}\left(U_{z}^{(e+m)}\right) \rightarrow D_{r_{m}}\left(U_{z}^{(e)}\right)
$$

which is an isometry between Banach algebras. A basis for this free extension is given by any choice of system of coset representatives for the finite group $U_{z}^{(e)} / U_{z}^{(e+m)}$.

Proof. Since $e \geq e_{\mathrm{uni}}$ each group $U_{z}^{(e)}$ is a uniform pro- $p$ group with lower $p$-series given by the subgroups $U_{z}^{(e+m)}$ for $m \geq 0$. The claim follows therefore from the discussion at the end of Section 2.2. 
Keep the assumptions of the preceding proposition and lemma. Put

$$
\mathcal{E}:=\mathcal{A}_{V} \# D_{r_{0}}\left(U_{z}^{(e+m)}\right) \quad \text { and } \quad \mathcal{E}^{\prime}:=\mathcal{A}_{V} \# D_{r_{m}}\left(U_{z}^{(e)}\right) .
$$

Consider the subsheaves $\mathfrak{n}^{\circ \text {,an }}$ and $\operatorname{ker} \lambda^{\circ \text {,an }}$ of the sheaf $\mathcal{O}_{X}$ an $\# U(\mathfrak{g})$ on $X^{\text {an }}$. Let $\mathcal{K}$ be the vector space of sections ${ }^{7}$ over the affinoid $V \subset X^{\text {an }}$ of one of these subsheaves. Put

$$
\mathcal{F}:=\mathcal{E} / \mathcal{K} \mathcal{E} \quad \text { and } \quad \mathcal{F}^{\prime}:=\mathcal{E}^{\prime} / \mathcal{K} \mathcal{E}^{\prime} .
$$

The ring homomorphism of the preceding lemma induces a ring homomorphism $\phi: \mathcal{E} \rightarrow \mathcal{E}^{\prime}$ and a linear homomorphism $\mathcal{F} \rightarrow \mathcal{F}^{\prime}$. The latter fits into a homomorphism

$$
\mathcal{F} \otimes_{D_{r_{0}}\left(U_{z}^{(e+m)}\right)} D_{r_{m}}\left(U_{z}^{(e)}\right) \rightarrow \mathcal{F}^{\prime}
$$

of $\left(\mathcal{F}, D_{r_{m}}\left(U_{z}^{(e)}\right)\right)$-bimodules.

Lemma 7.4.5. The rings $\mathcal{E}$ and $\mathcal{E}^{\prime}$ are noetherian. The homomorphism (7.4.4) is an isomorphism.

Proof. Since $\mathcal{A}_{V}$ is a noetherian ring, so is the ring $\mathcal{A}_{V} \# U(\mathfrak{g})$ [McConnell and Robson 1987, 1.7.14]. Choose generators $x_{1}, \ldots, x_{s}$ for the right $\mathcal{A}_{V} \# U(\mathfrak{g})$-ideal generated by the vector space $\mathcal{K}$. These generators determine a free presentation

$$
\bigoplus_{i=1, \ldots, s} \mathcal{E} \rightarrow \mathcal{E} \rightarrow \mathcal{F} \rightarrow 0
$$

of the right $\mathcal{E}$-module $\mathcal{F}$. The bijectivity of the natural map

$$
\mathcal{E} \otimes_{D_{r_{0}}\left(U_{z}^{(e+m)}\right)} D_{r_{m}}\left(U_{z}^{(e)}\right) \rightarrow \mathcal{E}^{\prime}, f \otimes h \mapsto \phi(f) \cdot h
$$

of $\left(\mathcal{E}, D_{r_{m}}\left(U_{z}^{(e)}\right)\right.$-bimodules can be checked on the level of vector spaces. It follows there from functoriality of $\mathcal{A}_{V} \hat{\otimes}_{L}(\cdot)$ applied to the obvious bijective linear map

$$
D_{r_{0}}\left(U_{z}^{(e+m)}\right) \otimes_{D_{r_{0}}\left(U_{z}^{(e+m)}\right)} D_{r_{m}}\left(U_{z}^{(e)}\right) \stackrel{\sim}{\longrightarrow} D_{r_{m}}\left(U_{z}^{(e)}\right) .
$$

Using 7.4.3 we conclude that $\mathcal{E}^{\prime}$ is a finite free left $\mathcal{E}$-module. By 7.4.1, the ring $\mathcal{E}$ is noetherian and so $\mathcal{E}^{\prime}$ is left noetherian. A similar argument shows that $\mathcal{E}^{\prime}$ is right noetherian. Finally, $\mathcal{E}$ is a right $D_{r_{0}}\left(U_{z}^{(e+m)}\right)$-module in the obvious way. Applying the functor $(\cdot) \otimes_{D_{r_{0}}\left(U_{z}^{(e+m)}\right)} D_{r_{m}}\left(U_{z}^{(e)}\right)$ to the above presentation yields the isomorphism

$$
\mathcal{F} \otimes_{D_{r_{0}}\left(U_{z}^{(e+m)}\right)} D_{r_{m}}\left(U_{z}^{(e)}\right) \simeq \mathcal{E}^{\prime} / \mathcal{K} \mathcal{E}^{\prime}=\mathcal{F}^{\prime}
$$

${ }^{7}$ Of course, $V$ is not an open subset of the topological space $X^{\text {an }}$. However, all sheaves in fact extend to sheaves with respect to the Grothendieck topology on the analytic space $X^{\text {an }}$. This technical point is of minor importance. 
We keep our assumptions: $z \in \mathscr{B}$ is a point and $V$ is a strictly affinoid neighborhood of $z$ on which $U_{z}^{(e-1)}$ acts analytically. Let $Y_{i} \subseteq V$ be finitely many affinoid domains such that the completed skew group ring $\mathcal{A}_{Y_{i}} \# D_{r_{0}}\left(U_{z}^{(e)}\right)$ exist. If $Y=\cap_{i} Y_{i}$ and $Y^{\prime}=\bigcup_{i} Y_{i}$, then one may verify that the skew group rings $\mathcal{A}_{Y} \# D_{r_{0}}\left(U_{z}^{(e)}\right)$ and $\mathcal{A}_{Y^{\prime}} \# D_{r_{0}}\left(U_{z}^{(e)}\right)$ exist as well. Indeed, since $V$ is separated, the case of $Y$ is straightforward and the case of $Y^{\prime}$ follows from considering a short exact sequence as in the proof of Corollary 6.4.4(ii). For any affinoid domain $Y \subseteq V$ such that the skew group ring $\mathcal{A}_{Y} \# D_{r_{0}}\left(U_{z}^{(e)}\right)$ exists, we have inside this ring the right ideal generated by $\mathfrak{n}^{\circ \text { an }}(Y)$. Such affinoid domains $Y$ together with finite coverings form a $G$-topology on $V$ and we may consider the sheaf $\mathcal{I}_{\mathfrak{t}}^{\text {an } z}$ associated to the presheaf

$$
Y \mapsto \mathfrak{n}^{\circ, \text { an }}(Y) \cdot \mathcal{A}_{Y} \# D_{r_{0}}\left(U_{z}^{(e)}\right)
$$

on $V$. It follows from Lemma 6.2.6 that we have for its stalk at $z$, that

$$
\left(\mathcal{I}_{\mathfrak{t}}^{\text {an }, z}\right)_{z}=\mathfrak{n}_{z}^{\circ, \text { an }} \cdot \mathcal{O}_{\mathscr{B}, z} \# D_{r_{0}}\left(U_{z}^{(e)}\right)=\mathscr{I}_{\mathfrak{t}, z}^{\text {an }}
$$

with the ideal sheaf $\mathscr{I}_{t}^{\text {an }} \subseteq \mathcal{O}_{\mathscr{B}} \# \underline{D}_{r_{0}}$. There is an analogous sheaf $\mathcal{I}_{\chi}^{\text {an, } z}$ on $V$ defined by replacing $\mathfrak{n}^{\circ}$ with $\operatorname{ker} \lambda^{\circ}$.

Finally, fix once and for all a neighborhood basis of $z$ consisting of strict affinoids $V^{\prime} \subset V$ on which $U_{z}^{(e-1)}$ acts analytically (Lemma 6.2.6). We give $\mathcal{O}_{\mathscr{B}, z} \# D_{r}\left(U_{z}^{(e)}\right)$ the inductive limit topology from the isomorphism (6.4.9) via transport of structure.

Corollary 7.4.6. Let $r=r_{m}$ for some $m \geq 0$ and keep the previous assumptions and notations.

(1) The isomorphism (5.1.6) induces an isometric isomorphism of Banach spaces

$$
\left(\mathcal{A}_{V} \# D_{r_{0}}\left(U_{z}^{(e,-)}\right)\right) \hat{\otimes}_{L} D_{r_{0}}\left(U_{z}^{(e, t)}\right) \stackrel{\sim}{\longrightarrow}\left(\mathcal{A}_{V} \# D_{r_{0}}\left(U_{z}^{(e)}\right)\right) / \mathcal{I}_{\mathfrak{t}}^{\text {an }, z}(V) .
$$

Here $U_{z}^{(e,-)}$ and $U_{z}^{(e, t)}$ are respectively the negative and toral parts in the root space decomposition of the group $U_{z}^{(e)}$ appearing in Proposition 4.1.7.

(2) The isomorphism (1) induces an isometric isomorphism of Banach spaces

$$
\mathcal{A}_{V} \# D_{r_{0}}\left(U_{z}^{(e,-)}\right) \stackrel{\sim}{\longrightarrow}\left(\mathcal{A}_{V} \# D_{r_{0}}\left(U_{z}^{(e)}\right)\right) / \mathcal{I}_{\chi}^{\mathrm{an}, z}(V) .
$$

(3) The ideals $\mathscr{I}_{\mathfrak{t}, z}^{\text {an }}$ and $\mathscr{I}_{\chi, z}^{\text {an }}$ are closed in $\mathcal{O}_{\mathscr{B}, z} \# D_{r}\left(U_{z}^{(e)}\right)$ (in the inductive limit topology).

Proof. Let us first assume $K=\mathbb{Q}_{p}$. Ad (1): We begin by introducing certain integral structures in the situation of 5.1.5: let $\mathfrak{B} \subset \mathfrak{G}$ be a Borel subgroup scheme over $\mathbb{Z}_{p}$ with generic fiber $\boldsymbol{B}$ and containing the Néron model $\mathfrak{T}:=\operatorname{Spec}\left(\mathbb{Z}_{p}\left[X^{*}(\boldsymbol{T})\right]\right)$ of $\boldsymbol{T}$. Denote the unipotent radical of $\mathfrak{B}$ by $\mathfrak{N}$. Let $\mathfrak{X}:=\mathfrak{G} / \mathfrak{B}$. The group scheme $\mathfrak{G}$ acts on $\mathfrak{X}$ by left translations and we have a derived action of its Lie algebra $\mathfrak{g}_{\mathbb{Z}_{p}}$ on $\mathfrak{X}$. Let $\mathfrak{N}^{-}$be the unipotent radical of the Borel subgroup scheme opposite to 
$\mathfrak{B}$. We denote by $\mathfrak{U}_{1}=q\left(\mathfrak{N}^{-}\right)$the image of $\mathfrak{N}^{-}$under the natural projection map $q: \mathfrak{G} \rightarrow \mathfrak{X}$. For each $w \in W$, we fix a representative $\dot{w}$ in $\mathfrak{G}\left(\mathbb{Z}_{p}\right)$ with $\dot{1}=1$ and put $\mathfrak{U}_{w}:=\dot{w} \mathfrak{U}_{1}$. The $\mathfrak{U}_{w}, w \in W$ form a Zariski covering of $\mathfrak{X}$ and each $\mathfrak{U}_{w}$ has generic fiber $U_{w}$.

If $U_{w}^{\text {an }}$ denotes the rigid analytification of $U_{w}$ and $U_{w}^{\text {an, }, 0}$ denotes the Raynaud generic fiber of $\mathfrak{U}_{w}$, then there is a natural morphism

$$
U_{w}^{\mathrm{an}, 0} \hookrightarrow U_{w}^{\mathrm{an}}
$$

identifying the source with an affinoid subdomain in the target. Let $\mathcal{B}_{w}$ be the affinoid algebra of $U_{w}^{\text {an, }, 0}$. Put

$$
V_{w}:=V \cap U_{w}^{\mathrm{an}, 0} .
$$

The $V_{w}, w \in W$ form a finite admissible affinoid covering of $V$. Let $V_{w w^{\prime}}:=V_{w} \cap V_{w^{\prime}}$ for $w, w^{\prime} \in W$ and denote by $\mathcal{A}_{w}$ and $\mathcal{A}_{w w^{\prime}}$ the affinoid algebras corresponding to $V_{w}$ and $V_{w w^{\prime}}$ respectively. We have a commutative diagram of restriction maps

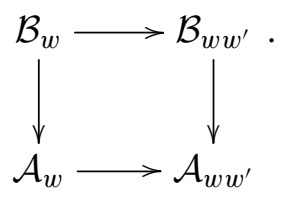

After these preliminary remarks we establish the isomorphism in (1) in several steps. Note that $G$-equivariance reduces us to proving the statement in the case where $z$ is contained in the closure $\overline{\mathscr{C}}$ of the fundamental chamber $\mathscr{C}$. So let $z \in \overline{\mathscr{C}}$ in the following. We follow the notation of the proof of Proposition 7.4.1 and denote by $\mathcal{L}(\cdot)$ the $\mathbb{Z}_{p}$-Lie algebra of a uniform pro- $p$ group. In particular,

$$
\mathfrak{h}_{\mathbb{Z}_{p}}:=\mathcal{L}\left(U_{z}^{(e-1)}\right), \quad \mathfrak{h}_{\overline{\mathbb{Z}}_{p}}^{-}:=\mathcal{L}\left(U_{z}^{(e-1,-)}\right) .
$$

Let $\hat{U}\left(\mathfrak{h}_{\mathbb{Z}_{p}}\right)$ and $\hat{U}\left(\mathfrak{h}_{\mathbb{Z}_{p}}^{-}\right)$denote the $p$-adic completions of the universal enveloping algebras of these Lie algebras. Note that

$$
\hat{U}\left(\mathfrak{h}_{\mathbb{Z}_{p}}\right) \otimes_{\mathbb{Z}_{p}} \mathbb{Q}_{p}=D_{r_{0}}\left(U_{z}^{(e)}\right), \quad \hat{U}\left(\mathfrak{h}_{\mathbb{Z}_{p}}^{-}\right) \otimes_{\mathbb{Z}_{p}} \mathbb{Q}_{p}=D_{r_{0}}\left(U_{z}^{(e,-)}\right) .
$$

Since $e>e_{\text {uni }}$ we have

$$
U_{z}^{(e-1)} \subseteq U_{w x_{0}}^{(0)}
$$

for all $w \in W$; see Remark 4.3.4. We fix in the following two elements $w, w^{\prime} \in W$. Note that $U_{w x_{0}}^{(0)}$ acts analytically on the affinoid domain $U_{w}^{\text {an, }, 0}$ and therefore so does $U_{z}^{(e-1)}$. Hence, $U_{z}^{(e-1)}$ acts analytically on $V_{w}$ and $V_{w w^{\prime}}$. From the proof of Proposition 7.4.1 we know that the induced action of the Lie algebra $\mathfrak{h}_{\mathbb{Z}_{p}}$ stabilizes the subrings $\mathcal{A}_{V}^{\circ}, \mathcal{A}_{w}^{\circ}$ and $\mathcal{A}_{w w^{\prime}}^{\circ}$ of power-bounded elements in $\mathcal{A}_{V}, \mathcal{A}_{w}$ and $\mathcal{A}_{w w^{\prime}}$ respectively. To simplify notation, we denote in the following by $\mathcal{A}^{\circ}$ one of the 
rings $\mathcal{A}_{V}^{\circ}, \mathcal{A}_{w}^{\circ}$ or $\mathcal{A}_{w w^{\prime}}^{\circ}$ and let $\mathcal{A}:=\mathcal{A}^{\circ} \otimes_{\mathbb{Z}_{p}} \mathbb{Q}_{p}$. The affinoid space $\mathscr{M}(\mathcal{A})$ equals therefore one of the affinoid domains $V, V_{w}$ or $V_{w w^{\prime}}$ of $V$. As we have just seen, the space of sections $\mathcal{I}_{\mathfrak{t}}^{\text {an, } z}(\mathscr{M}(\mathcal{A}))$ is defined.

The root space decomposition of $U_{z}^{(e-1)}$ (Proposition 4.1.7) induces a decomposition

$$
\mathfrak{h}_{\mathbb{Z}_{p}}=\mathfrak{h}_{\mathbb{\mathbb { Z }}_{p}}^{-} \oplus \mathfrak{h}_{\mathbb{Z}_{p}}^{t} \oplus \mathfrak{h}_{\mathbb{Z}_{p}}^{+}
$$

which upon tensoring with $\mathbb{Q}_{p}$ gives the triangular decomposition $\mathfrak{g}=\mathfrak{n}^{-} \oplus \mathfrak{t} \oplus \mathfrak{n}^{+}$ of the reductive Lie algebra $\mathfrak{g}$. Let

$$
\iota: U\left(\mathfrak{h}_{\mathbb{Z}_{p}}^{-}\right) \otimes_{\mathbb{Z}_{p}} U\left(\mathfrak{h}_{\mathbb{Z}_{p}}^{t}\right) \hookrightarrow U\left(\mathfrak{h}_{\mathbb{Z}_{p}}\right)
$$

be the linear PBW-map induced from this decomposition and form the linear map

$$
f:\left(\mathcal{A}^{\circ} \# U\left(\mathfrak{h}_{\mathbb{Z}_{p}}^{-}\right)\right) \otimes_{\mathbb{Z}_{p}} U\left(\mathfrak{h}_{\mathbb{Z}_{p}}^{t}\right) \rightarrow\left(\mathcal{A}^{\circ} \# U\left(\mathfrak{h}_{\mathbb{Z}_{p}}\right)\right),(f \otimes \mathfrak{x}) \otimes \mathfrak{y} \mapsto f \otimes \iota(\mathfrak{x} \otimes \mathfrak{y}) .
$$

Let $\hat{f}$ be the $p$-adic completion of the map $f$. Composing $\hat{f} \otimes_{\mathbb{Z}_{p}} \mathbb{Q}_{p}$ with the natural projection map

$$
\mathcal{A} \# D_{r_{0}}\left(U_{z}^{(e)}\right) \rightarrow\left(\mathcal{A} \# D_{r_{0}}\left(U_{z}^{(e)}\right)\right) / \mathcal{I}_{\mathfrak{t}}^{\mathrm{an}, z}(\mathscr{M}(\mathcal{A}))
$$

yields a linear map

$$
\psi_{\mathcal{A}}:\left(\mathcal{A} \# D_{r_{0}}\left(U_{z}^{(e,-)}\right)\right) \hat{\otimes}_{\mathbb{Q}_{p}} D_{r_{0}}\left(U_{z}^{(e, t)}\right) \rightarrow\left(\mathcal{A} \# D_{r_{0}}\left(U_{z}^{(e)}\right)\right) / \mathcal{I}_{\mathfrak{t}}^{\mathrm{an}, z}(\mathscr{M}(\mathcal{A})) .
$$

According to Proposition 7.4.1, the Banach algebra $\mathcal{A} \# D_{r_{0}}\left(U_{z}^{(e)}\right)$ is noetherian, and hence, its right ideal $\mathcal{I}_{\mathrm{t}}^{\text {an, } z}(\mathscr{M}(\mathcal{A}))$ is closed. If we endow the target of $\psi_{\mathcal{A}}$ with the quotient norm, then $\psi_{\mathcal{A}}$ becomes a norm-decreasing linear map between Banach spaces. In the case where $\mathcal{A}=\mathcal{A}_{V}$, i.e., $\mathscr{M}(\mathcal{A})=V$, we denote this map by $\psi_{V}$. We claim that $\psi_{V}$ is our searched for isomorphism appearing in (1). To prove this we will show, as a first step, that the map $\psi_{\mathcal{A}}$ is an isometric isomorphism of Banach spaces for $\mathcal{A}$ equal to one of the rings $\mathcal{A}_{w}$ or $\mathcal{A}_{w w^{\prime}}$. We will do this with the help of auxiliary isomorphisms coming from [Ardakov and Wadsley 2013] and involving the "congruence group" $U_{w x_{0}}^{(0)}$. So suppose that $\mathcal{A}$ is either $\mathcal{A}_{w}$ or $\mathcal{A}_{w w^{\prime}}$, so that $\mathscr{M}(\mathcal{A}) \subseteq U_{w}^{\mathrm{an}, 0}$. We may apply essentially the same construction above to the algebra $\mathcal{A}$ and the group $U_{w x_{0}}^{(0)}$ and obtain from [loc. cit.] an isometric isomorphism of Banach spaces

$$
\psi_{\mathcal{A}}^{0}:\left(\mathcal{A} \# D_{r_{0}}\left(U_{w x_{0}}^{(0,-)}\right)\right) \hat{\otimes}_{L} D_{r_{0}}\left(U_{w x_{0}}^{(0, t)}\right) \rightarrow\left(\mathcal{A} \# D_{r_{0}}\left(U_{w x_{0}}^{(0)}\right)\right) / J_{\mathcal{A}},
$$

$J_{\mathcal{A}}$ being the right ideal induced by $\mathfrak{n}^{\circ \text {,an }}(\mathscr{M}(\mathcal{A}))$. Let us explain this isomorphism in more detail. Since $U_{x_{0}}^{(0)}=\mathfrak{G}(p)$, we have $\mathcal{L}\left(U_{w x_{0}}^{(0)}\right)=p \mathfrak{g}_{\mathbb{Z}_{p}}^{w}$ as $\mathbb{Z}_{p}$-Lie algebras where $\mathfrak{g}_{\mathbb{Z}_{p}}^{w}:=\operatorname{Ad}(\dot{w})\left(\mathfrak{g}_{\mathbb{Z}_{p}}\right)$. In particular, $D_{r_{0}}\left(U_{w x_{0}}^{(0)}\right)$ equals the $p$-adic completion (with $p$ inverted) of the universal enveloping algebra $U\left(\mathfrak{g}_{\mathbb{Z}_{p}}^{w}\right)_{1}:=U\left(p \mathfrak{g}_{\mathbb{Z}_{p}}^{w}\right)$. Our 
sheaf $\mathcal{D}_{\mathfrak{t}}$ on $X$, as introduced in 5.1.5, equals the pull-back along the inclusion map $X \subset \mathfrak{X}$ of the relative enveloping algebra

$$
\tilde{\mathcal{D}}:=\xi_{*}\left(\mathcal{D}_{\tilde{\mathfrak{X}}}\right)^{\mathfrak{H}}
$$

of the locally trivial $\mathfrak{H}$-torsor

$$
\xi: \tilde{\mathfrak{X}} \rightarrow \mathfrak{X}
$$

appearing in [loc. cit., 4.7]. Here, $\tilde{\mathfrak{X}}$ denotes the homogeneous space $\mathfrak{G} / \mathfrak{N}$ with ring of crystalline level zero differential operators $\mathcal{D}_{\tilde{\mathfrak{X}}}$, the symbol $\mathfrak{H}$ denotes the abstract Cartan group $\mathfrak{B} / \mathfrak{N}$ and $\xi$ equals the map $g \mathfrak{N} \mapsto g \mathfrak{B}$. In this situation, the map $\psi_{\mathcal{A}}^{0}$ and its properties follow from [loc. cit., Lemma 6.4(a)] and its proof like this: in the notation of [loc. cit.] choose $n=1$ and put $U:=\mathfrak{U}_{w}$ or $\mathfrak{U}_{w} \cap \mathfrak{U}_{w^{\prime}}$ depending on $\mathcal{A}=\mathcal{A}_{w}$ or $\mathcal{A}_{w w^{\prime}}$ respectively. Since $U$ trivializes the torsor $\xi$, we have the isomorphism

$$
\left.\left.\left(\mathcal{D}_{1}\right)\right|_{U} \otimes_{\mathbb{Z}_{p}} U\left(\mathfrak{t}_{\mathbb{Z}_{p}}\right)_{1} \simeq\left(\tilde{\mathcal{D}}_{1}\right)\right|_{U}
$$

of sheaves of $\mathbb{Z}_{p}$-algebras. Here, $\mathcal{D}$ denotes the sheaf of "crystalline level zero" differential operators on $\mathfrak{X}$, the subscript $(\cdot)_{1}$ refers to the first deformation functor of [loc. cit.] and we have identified the Lie algebra of the $\mathbb{Z}_{p}$-group scheme $\mathfrak{H}$ with $\mathfrak{t}_{\mathbb{Z}_{p}}:=\operatorname{Lie}(\mathfrak{T})$ via the morphism $\mathfrak{T} \subset \mathfrak{B} \rightarrow \mathfrak{H}$. Let $\mathfrak{n}_{\mathbb{Z}_{p}}:=\operatorname{Lie}\left(\mathfrak{N}^{-}\right)$. Then

$$
U\left(\mathfrak{t}_{\mathbb{Z}_{p}}\right)_{1}=U\left(p \mathfrak{t}_{\mathbb{Z}_{p}}\right)=U\left(\mathcal{L}\left(U_{x_{0}}^{(0, t)}\right)\right)
$$

and

$$
U\left(\mathfrak{n}_{\mathbb{Z}_{p}}^{w}\right)_{1}=U\left(p \mathfrak{n}_{\mathbb{Z}_{p}}^{w}\right)=U\left(\mathcal{L}\left(U_{w x_{0}}^{(0,-)}\right)\right)
$$

as $\mathbb{Z}_{p}$-subalgebras inside the $\mathbb{Q}_{p}$-algebra $U(\mathfrak{g})$. The above isomorphism of sheaves extends to an isomorphism

$$
\left.\left.\left(\widehat{\left(\mathcal{D}_{1}\right)}\right)\right|_{U} \hat{\otimes}_{\mathbb{Z}_{p}} \widehat{U\left(\mathfrak{t}_{\mathbb{Z}_{p}}\right)}\right)\left.\simeq\left(\widehat{\left(\tilde{\mathcal{D}}_{1}\right)}\right)\right|_{U}
$$

involving the $p$-adic completions of the former sheaves. We may view these sheaves as sheaves on the formal scheme $\operatorname{Spf} \mathcal{A}^{\circ}$. Taking global sections and subsequent inversion of $p$ yields an isometric isomorphism of Banach spaces

$$
\left(\mathcal{A} \# D_{r_{0}}\left(U_{w x_{0}}^{(0,-)}\right)\right) \hat{\otimes}_{\mathbb{Q}_{p}} D_{r_{0}}\left(U_{w x_{0}}^{(0, t)}\right) \rightarrow\left(\mathcal{A} \# D_{r_{0}}\left(U_{w x_{0}}^{(0)}\right)\right) / J_{\mathcal{A}}
$$

with, as already indicated, $J_{\mathcal{A}}$ equal to the right ideal induced by $\mathfrak{n}^{\circ \text {,an }}(\mathscr{M}(\mathcal{A}))$. This is our promised isomorphism $\psi_{\mathcal{A}}^{0}$. Note that there is a canonical map

$$
\mathcal{I}_{\mathfrak{t}}^{\mathrm{an}, z}(\mathscr{M}(\mathcal{A})) \rightarrow J_{\mathcal{A}}
$$

induced from the inclusion $D_{r_{0}}\left(U_{z}^{(e)}\right) \rightarrow D_{r_{0}}\left(U_{w x_{0}}^{(0)}\right)$. 
The maps $\psi_{\mathcal{A}}$ and $\psi_{\mathcal{A}}^{0}$ fit into the diagram

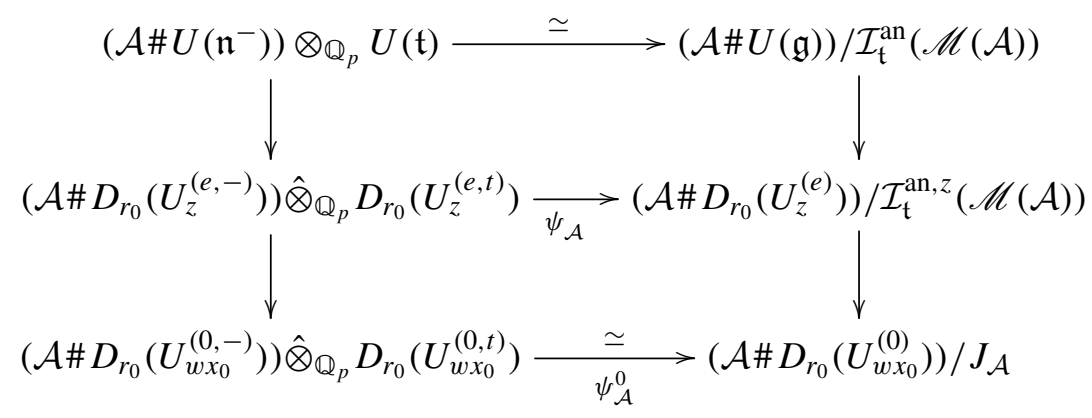

where the top horizontal arrow is the pull-back along the canonical morphism

$$
\mathscr{M}(\mathcal{A}) \subset U_{w}^{\text {an }} \rightarrow U_{w}
$$

of the isomorphism (5.1.6) and therefore an algebra isomorphism itself. The bottom vertical arrows are induced by the inclusion $U_{z}^{(e)} \subseteq U_{w x_{0}}^{(0)}$.

The group $U_{z}^{(e,-)}$ is an open subgroup of the $p$-adic group $N^{-}\left(\mathbb{Q}_{p}\right)$. Applying $D_{r_{0}}(\cdot)$ yields therefore a completion of $U\left(\mathfrak{n}^{-}\right)$. Similarly, applying $D_{r_{0}}(\cdot)$ to the groups $U_{z}^{(e, t)}$ and $U_{z}^{(e)}$ yields a completion of $U(\mathfrak{t})$ and $U(\mathfrak{g})$ respectively. These completions define the top vertical arrows. Unwinding the definitions of all the maps involved shows that the diagram commutes. The bottom left vertical arrow obviously is injective. Since $\psi_{\mathcal{A}}^{0}$ is bijective, the commutativity of the lower square implies $\psi_{\mathcal{A}}$ to be injective. For its surjectivity, consider the inverse map of the upper horizontal isomorphism. It is induced from the PBW-projection $\mathfrak{g} \rightarrow \mathfrak{n}^{-} \oplus \mathfrak{t}$. The corresponding projection map arising from the triangular decomposition of $\mathfrak{h}_{\mathbb{Z}_{p}}$ completes to a linear map

$$
\left(\mathcal{A} \# D_{r_{0}}\left(U_{z}^{(e)}\right)\right) \rightarrow\left(\mathcal{A} \# D_{r_{0}}\left(U_{z}^{(e,-)}\right)\right) \hat{\otimes}_{\mathbb{Q}_{p}} D_{r_{0}}\left(U_{z}^{(e, t)}\right),
$$

which factors through the target of $\psi_{\mathcal{A}}$ and gives a section to $\psi_{\mathcal{A}}$. All in all, we have shown that $\psi_{\mathcal{A}}$ is an isometric isomorphism between Banach spaces. This completes our first step.

In a second step, we show that the map $\psi_{V}$ is an isometric isomorphism of Banach spaces. We put $\psi_{V_{w}}:=\psi_{\mathcal{A}}$ in case $\mathcal{A}=\mathcal{A}_{w}$ and $\psi_{V_{w w^{\prime}}}:=\psi_{\mathcal{A}}$ in case $\mathcal{A}=\mathcal{A}_{w w^{\prime}}$. Abbreviate

$$
D:=D_{r_{0}}\left(U_{z}^{(e)}\right), \quad D^{t}:=D_{r_{0}}\left(U_{z}^{(e, t)}\right), \quad D^{-}:=D_{r_{0}}\left(U_{z}^{(e,-)}\right) .
$$

The covering $V=\bigcup_{w \in W} V_{w}$ gives rise to the exact restriction sequence

$$
0 \rightarrow \mathcal{A}_{V} \rightarrow \bigoplus_{w} \mathcal{A}_{w} \rightarrow \underset{w<w^{\prime}}{\bigoplus} \mathcal{A}_{w w^{\prime}}
$$


where we have ordered the elements of $W$ in some arbitrary way. It induces two complexes, namely

$$
0 \rightarrow\left(\mathcal{A}_{V} \# D^{-}\right) \hat{\otimes} D^{t} \rightarrow \bigoplus_{w}\left(\mathcal{A}_{w} \# D^{-}\right) \hat{\otimes} D^{t} \rightarrow \underset{w<w^{\prime}}{\bigoplus}\left(\mathcal{A}_{w w^{\prime}} \# D^{-}\right) \hat{\otimes} D^{t}
$$

and

$$
\begin{aligned}
0 \rightarrow\left(\mathcal{A}_{V} \# D\right) / \mathcal{I}_{\mathfrak{t}}^{\mathrm{an}, z}(V) & \rightarrow \bigoplus_{w}\left(\mathcal{A}_{w} \# D\right) / \mathcal{I}_{\mathfrak{t}}^{\mathrm{an}, z}\left(V_{w}\right) \\
& \rightarrow \bigoplus_{w<w^{\prime}}\left(\mathcal{A}_{w w^{\prime}} \# D\right) / \mathcal{I}_{\mathfrak{t}}^{\mathrm{an}, z}\left(V_{w w^{\prime}}\right) .
\end{aligned}
$$

The maps $\psi_{V}, \psi_{V_{w}}$ and $\psi_{V_{w w^{\prime}}}$ induce a morphism between (I) and (II). We claim that (I) is exact. Indeed, exactness may be shown on the level of vector spaces. So let $d$ be the rank of the finitely generated free $\mathbb{Z}_{p}$-module $\mathfrak{h}_{\mathbb{Z}_{p}}^{-} \oplus \mathfrak{h}_{\mathbb{Z}_{p}}^{t}$. By construction, the Banach space $D^{-} \hat{\otimes} D^{t}$ is isomorphic to the Banach space underlying the Tate algebra of the $d$-dimensional closed unit disc $B$. Our assertion follows now from the sheaf property of $\mathcal{O}_{V \times{ }_{K} B}$ applied to the admissible covering $V \times{ }_{K} B=\bigcup_{w} V_{w} \times_{K} B$. Since the maps $\psi_{V_{w}}$ are all isomorphisms by our first step, it follows that $\psi_{V}$ is injective. To establish its surjectivity, observe that the second arrow in (II),

$$
\left(\mathcal{A}_{V} \# D\right) / \mathcal{I}_{\mathfrak{t}}^{\text {an, } z}(V) \rightarrow \bigoplus_{w}\left(\mathcal{A}_{w} \# D\right) / \mathcal{I}_{\mathfrak{t}}^{\text {an }, z}\left(V_{w}\right),
$$

is injective. This follows from an easy diagram chase in

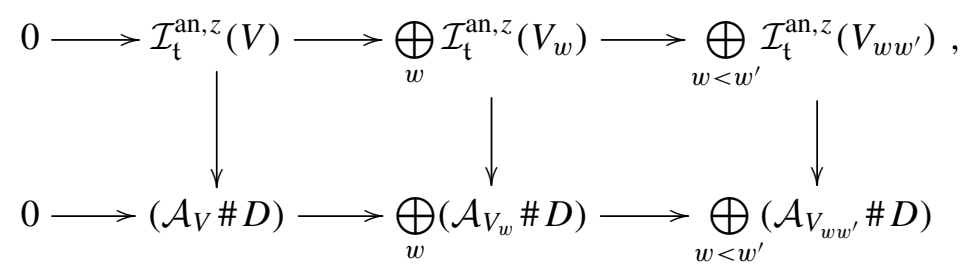

where we have exact rows and injective vertical maps. Since all the maps $\psi_{V_{w}}$ and $\psi_{V_{w_{w}}}$ are isomorphisms by our first step and since (II) is a complex, this implies the surjectivity of $\psi_{V}$. Then $\psi_{V}$ must be an isometric isomorphism of Banach spaces. This completes the proof of (1). Treating the ideal $\mathcal{I}_{\chi}^{\text {an, } z}$ in the same way gives (2). Ad (3): By $G$-equivariance we may assume that $z$ is contained in the closure of the fundamental chamber $\mathscr{C}$. Recall our fixed choice of neighborhood basis consisting of strict affinoids $V \subset X^{\text {an }}$ on which $U_{z}^{(e-1)}$ acts analytically. Since the isomorphism in (1) is compatible with the restriction maps $\mathcal{A}_{V} \rightarrow \mathcal{A}_{V^{\prime}}$ associated to an inclusion $V^{\prime} \subset V$ we see that Proposition 2.4.1 applies to the locally convex inductive limit

$$
\underset{V}{\lim _{\vec{V}}} \mathcal{A}_{V} \# D_{r_{0}}\left(U_{z}^{(e)}\right) / \mathcal{I}_{\mathrm{t}}^{\mathrm{an}, z}(V) .
$$

The limit is therefore Hausdorff. Moreover, the isomorphism (7.4.4) appearing in Lemma 7.4.5 is also compatible with the map $\mathcal{A}_{V} \rightarrow \mathcal{A}_{V^{\prime}}$. Since a finite direct 
sum of Hausdorff spaces is again Hausdorff, Lemma 7.4.3 implies that the locally convex inductive limit

$$
\underset{V}{\lim _{\longrightarrow}} \mathcal{A}_{V} \# D_{r_{m}}\left(U_{z}^{(e)}\right) / \mathcal{I}_{\mathrm{t}, m}^{\mathrm{an}, z}(V)
$$

is Hausdorff. Here, the sheaf $\mathcal{I}_{\mathfrak{t}, m}^{\text {an }, z}$ is defined by replacing in the definition of $\mathcal{I}_{\mathfrak{t}}^{\text {an }, z}$ the ring $D_{r_{0}}\left(U_{z}^{(e)}\right)$ by its subring $D_{r_{m}}\left(U_{z}^{(e)}\right)$. We may now finish the proof of (3). Proposition 2.4.1 gives a (topological) linear isomorphism

$$
\mathcal{O}_{\mathscr{B}, z} \# D_{r_{m}}\left(U_{z}^{(e)}\right) \simeq \underset{V}{\lim } \mathcal{A}_{V} \# D_{r_{m}}\left(U_{z}^{(e)}\right) .
$$

By Lemma 6.2.6 we have

$$
\mathscr{I}_{\mathfrak{t}, z}^{\text {an }}=\underset{V}{\lim } \mathcal{I}_{\mathfrak{t}, m}^{\text {an }, z}(V)
$$

for the ideal sheaf $\mathscr{I}_{\mathfrak{t}}^{\text {an }} \subseteq \mathcal{O}_{\mathscr{B}} \# \underline{D}_{r_{m}}$. Consider the diagram of continuous $K$-linear maps

$0 \longrightarrow \underset{V}{\lim } \mathcal{I}_{\mathfrak{t}, m}^{\text {an, } z}(V) \stackrel{\iota}{\longrightarrow} \underset{V}{\lim } \mathcal{A}_{V} \# D_{r_{m}}\left(U_{z}^{(e)}\right) \longrightarrow \underset{V}{\lim } \mathcal{A}_{V} \# D_{r_{m}}\left(U_{z}^{(e)}\right) / \mathcal{I}_{\mathfrak{t}, m}^{\text {an, } z}(V) \longrightarrow 0$,

which is short exact as a diagram of abstract $K$-vector spaces. The right-hand term is Hausdorff, as we have just seen. The injection $\iota$ has therefore closed image which is what we want. The case of the ideal $\mathscr{I}_{\chi, z}^{\text {an }}$ follows similarly by using the sheaf $\mathcal{I}_{\chi}^{\text {an, } z}$ and the isomorphism (2). This finishes the proof of the corollary in the case $K=\mathbb{Q}_{p}$. A base change along the finite field extension $\mathbb{Q}_{p} \subseteq K$ yields the general case.

We emphasize that the top vertical arrows in the commutative diagram ( $\dagger$ ) appearing in the preceding proof are injective and have dense image. Moreover, the top horizontal arrow is multiplicative. In particular, if the target of $\psi_{\mathcal{A}}$ were a ring, i.e., if the right ideal $\mathcal{I}_{\mathfrak{t}}^{\text {an, } z}(\mathscr{M}(\mathcal{A}))$ were two-sided, $\psi_{\mathcal{A}}$ would be a ring homomorphism.

Lemma 7.4.7. Let $r=r_{m}$ for some $m \geq 0$ and keep the previous assumptions and notations. The right ideals $\mathscr{I}_{t}^{\text {an }}$ and $\mathscr{I}_{\chi}^{\text {an }}$ are two-sided ideals. Let $z \in \mathscr{B}$. The isomorphism (1) of the preceding corollary induces an isomorphism of $K$-algebras

$$
\left(\mathcal{O}_{\mathscr{B}, z} \# D_{r_{0}}\left(U_{z}^{(e,-)}\right)\right) \hat{\otimes}_{L} D_{r_{0}}\left(U_{z}^{(e, t)}\right) \stackrel{\sim}{\longrightarrow}\left(\mathcal{O}_{\mathscr{B}, z} \# D_{r_{0}}\left(U_{z}^{(e)}\right)\right) / \mathscr{I}_{\mathbf{t}, z}^{\text {an }} .
$$

Similarly, the isomorphism (2) of the preceding corollary induces an isomorphism of $K$-algebras

$$
\mathcal{O}_{\mathscr{B}, z} \# D_{r_{0}}\left(U_{z}^{(e,-)}\right) \stackrel{\sim}{\longrightarrow}\left(\mathcal{O}_{\mathscr{B}, z} \# D_{r_{0}}\left(U_{z}^{(e)}\right)\right) / \mathscr{I}_{\chi, z}^{\text {an }} .
$$

Proof. According to Section 8.3 the sheaves $\mathcal{O}_{\mathscr{B}} \# U\left(\mathfrak{g}_{K}\right)$ and $\mathcal{O}_{\mathscr{B}} \# \underline{D_{r}}$ have a natural $G$-equivariant structure such that the morphism $\mathcal{O}_{\mathscr{B}} \# U\left(\mathfrak{g}_{K}\right) \rightarrow \mathcal{O}_{\mathscr{B}} \# \underline{D}_{r}$ is equivariant. Moreover, the ideals $\mathcal{I}_{\mathscr{B}, t}^{\text {an }}$ and $\mathcal{I}_{\mathscr{B}, \chi}^{\text {an }}$ are $G$-stable. Hence, so are the 
right ideals $\mathscr{I}_{t}^{\text {an }}$ and $\mathscr{I}_{\chi}^{\text {an }}$. That these ideals are two-sided can be checked stalkwise [Godement 1958, II.1.8]. We give the argument in the case $\mathscr{I}_{t}^{\text {an }}$. The other case follows in the same way. Recall that we have fixed a neighborhood basis of $z$ consisting of strict affinoids $V$ on which $U_{z}^{(e-1)}$ acts analytically. The corresponding inductive limit topology makes $\mathcal{O}_{\mathscr{B}, z} \# D_{r}\left(U_{z}^{(e)}\right)$ a separately continuous $K$-algebra and, hence, the multiplication map $D_{r}\left(U_{z}^{(e)}\right) \rightarrow \mathcal{O}_{\mathscr{B}, z} \# D_{r}\left(U_{z}^{(e)}\right), \lambda \mapsto \lambda \cdot \partial$ is continuous for every $\partial \in \mathcal{O}_{\mathscr{B}, z} \# D_{r}\left(U_{z}^{(e)}\right)$. Fix $\partial \in \mathscr{I}_{\mathfrak{t}, z}^{\text {an }}$. By [Schneider and Teitelbaum 2002, Lemma 3.1] and part (3) of the preceding corollary, we see that it suffices to prove that $\delta_{g} \cdot \partial \in \mathcal{O}_{\mathscr{B}, z} \# D_{r}\left(U_{z}^{(e)}\right)$ lies in the subspace $\mathscr{I}_{\mathrm{t}, z}^{\text {an }}$ for $g \in U_{z}^{(e)}$. Considering $\partial$ as an element of $\mathcal{O}_{\mathscr{B}, z} \# D_{r}\left(U_{z}^{(e)}\right)$ we may choose, by Proposition 2.4.1, an affinoid neighborhood $V$ in our fixed neighborhood basis of $z$, such that $\partial \in \mathcal{A}_{V} \# D_{r}\left(U_{z}^{(e)}\right)$. Using power series expansions for elements of completed distribution algebras (Section 2.2.3) we may write $\partial$ as an infinite sum $\partial=\sum_{\alpha \in \mathbb{N}_{0}^{d}} f_{\alpha} \hat{\otimes} \boldsymbol{b}^{\alpha}$, with $f_{\alpha} \in \mathcal{A}_{V}$ converging in the Banach algebra $\mathcal{A}_{V} \# D_{r}\left(U_{z}^{(e)}\right)$. By definition of the skew multiplication (3.2.2) we have

$$
\begin{aligned}
\delta_{g} \cdot \partial & =\sum_{\alpha \in \mathbb{N}_{0}^{d}}\left(g \cdot f_{\alpha}\right) \hat{\otimes} \delta_{g} \boldsymbol{b}^{\alpha}=\sum_{\alpha \in \mathbb{N}_{0}^{d}}\left(g \cdot f_{\alpha}\right) \hat{\otimes}\left(\delta_{g} \boldsymbol{b}^{\alpha} \delta_{g}^{-1}\right) \delta_{g} \\
& =\sum_{\alpha \in \mathbb{N}_{0}^{d}} g^{*}\left(f_{\alpha} \hat{\otimes} \boldsymbol{b}^{\alpha}\right) \delta_{g}=g^{*}(\partial) \delta_{g},
\end{aligned}
$$

which is an element of $\mathscr{I}_{t}^{\text {an }}(V)$. Here, $g^{*}: \mathscr{I}_{t}^{\text {an }}(V) \stackrel{\sim}{\longrightarrow} \mathscr{I}_{t}^{\text {an }}(V)$ is induced by the equivariant structure on the sheaf $\mathscr{I}_{\mathrm{t}}^{\text {an }}$ (note that $U_{z}^{(e)}$ acts analytically on $V$ ). Passing to the stalk we obtain $\delta_{g} \cdot \partial \in \mathscr{I}_{\mathfrak{t}, z}^{\text {an }}$ Thus, the right ideals $\mathscr{I}_{\mathfrak{t}}^{\text {an }}$ and $\mathscr{I}_{\chi}^{\text {an }}$ are indeed two-sided ideals. Let $z \in \mathscr{B}$. Passing the isomorphisms (1) and (2) of the preceding corollary to the inductive limit over a neighborhood basis of $z$ consisting of affinoids $V$ on which $U_{z}^{(e-1)}$ acts analytically gives linear isomorphisms between $K$-algebras which are actually multiplicative; see the remark directly after the proof of the corollary. Hence, the lemma is proved.

In the following we tacitly restrict to numbers $r$ of the form $r=r_{m}$ for some $m \geq 0$. By the preceding lemma we may form the quotient sheaves

$$
\mathscr{D}_{r, \mathrm{t}}:=\left(\mathcal{O}_{\mathscr{B}} \# \underline{D}_{r}\right) / \mathscr{I}_{\mathrm{t}}^{\text {an }}, \quad \mathscr{D}_{r, \chi}:=\left(\mathcal{O}_{\mathscr{B}} \# \underline{D}_{r}\right) / \mathscr{I}_{\chi}^{\text {an }} .
$$

These are sheaves of (noncommutative) $K$-algebras on $\mathscr{B}$ and, at the same time, $\mathcal{O}_{\mathscr{B}}$-modules. We have a commutative diagram of morphisms

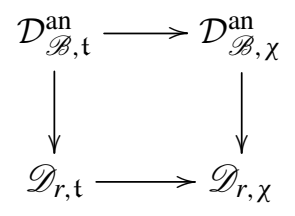


with surjective horizontal arrows. Moreover, it follows from (6.1.4) and the preceding lemma that the lower horizontal arrow induces an isomorphism

$$
\mathscr{D}_{r, t} /(\operatorname{ker} \lambda) \mathscr{D}_{r, \mathfrak{t}} \stackrel{\sim}{\longrightarrow} \mathscr{D}_{r, \chi} .
$$

We have the following extension of the property 2 in [Beilinson and Bernstein 1981, Section 2, Lemme].

Proposition 7.4.10. The morphism $\underline{D}_{r} \rightarrow \mathcal{O}_{\mathscr{B}} \# \underline{D}_{r} \rightarrow \mathscr{D}_{r, \chi}$ factors through $\underline{D}_{r} \rightarrow$ $\underline{D}_{r, \theta}$.

Proof. Letting $\mathscr{K}$ be the kernel of the morphism $\underline{D}_{r} \rightarrow \underline{D}_{r, \theta}$ the claim amounts to

$$
\mathscr{K} \subseteq \operatorname{ker}\left(\underline{D}_{r} \rightarrow \mathscr{D}_{r, \chi}\right) .
$$

This can be checked stalkwise; i.e., we are reduced to showing that, for each $z \in \mathscr{B}$ the natural map $D_{r}\left(U_{z}^{(e)}\right) \rightarrow \mathcal{O}_{\mathscr{B}, z} \# D_{r}\left(U_{z}^{(e)}\right) /\left(\mathscr{I}_{\chi}\right)_{z}$ factors through $D_{r}\left(U_{z}^{(e)}\right)_{\theta}$. The kernel of the map $D_{r}\left(U_{z}^{(e)}\right) \rightarrow D_{r}\left(U_{z}^{(e)}\right)_{\theta}$ is generated by

$$
I_{\theta}:=\operatorname{ker}\left(U\left(\mathfrak{g}_{K}\right) \rightarrow U\left(\mathfrak{g}_{K}\right)_{\theta}\right)
$$

and the ideal $\left(\mathscr{I}_{\chi}\right)_{z}$ is generated by the image of $\mathcal{I}_{\mathscr{B}, \chi, z}^{\text {an }}$. It therefore suffices to show that the natural map $U\left(\mathfrak{g}_{K}\right) \rightarrow \mathcal{O}_{\mathscr{B}, z} \# U\left(\mathfrak{g}_{K}\right)$ maps $I_{\theta}$ into $\mathcal{I}_{\mathscr{B}, \chi, z}^{\text {an }}$. This follows from [loc. cit.].

7.4.11. Let us finally make the structure of the stalks of the sheaves $\mathscr{D}_{r, \mathrm{t}}$ and $\mathscr{D}_{r, \chi}$ at a point $z \in \mathscr{B}$ more explicit. According to Lemma 6.2.2 the local $\operatorname{ring} \mathcal{O}_{\mathscr{B}, z}$ is a field. For simplicity we put $\kappa(z):=\mathcal{O}_{\mathscr{B}, z}$ and view this as a topological field of compact type. Note that the Berkovich point $z \in \mathscr{B} \subset X^{\text {an }}$ canonically induces a norm topology on $\kappa(z)$ which is weaker than our topology. We shall not make use of this norm topology in the following.

By [loc. cit.] together with Section 5.1.1 we furthermore have $\left(\mathfrak{n}^{\circ}\right)_{\pi(z)}=\mathfrak{n}_{\pi(z)}$ and $\left(\mathfrak{b}^{\circ}\right)_{\pi(z)}=\mathfrak{b}_{\pi(z)}$ for the stalks of the sheaves $\mathfrak{n}^{\circ}$ and $\mathfrak{b}^{\circ}$ at $\pi(z)=\eta$ (the generic point of $X$ ). Since passage to the stalk is exact, this proves the following lemma. It gives back the isomorphisms of Lemma 7.4.7 in case $r=r_{0}$.

Lemma 7.4.12. Assume $r=r_{m}$ for some $m \geq 0$. Let $z \in \mathscr{B}$. There is a canonical isomorphism

$$
\mathscr{D}_{r, \mathfrak{t}, z} \stackrel{\sim}{\longrightarrow}\left(\kappa(z) \hat{\otimes}_{L} D_{r}\left(U_{z}^{(e)}\right)\right) / \mathfrak{n}_{\pi(z)}\left(\kappa(z) \hat{\otimes}_{L} D_{r}\left(U_{z}^{(e)}\right)\right) .
$$

This isomorphism induces a canonical isomorphism between $\mathscr{D}_{r, \chi, z}$ and the $\lambda$ coinvariants of the $\mathfrak{t}_{K}$-module $\left(\kappa(z) \hat{\otimes}_{L} D_{r}\left(U_{z}^{(e)}\right)\right) / \mathfrak{n}_{\pi(z)}\left(\kappa(z) \hat{\otimes}_{L} D_{r}\left(U_{z}^{(e)}\right)\right)$. In particular,

$$
\mathscr{D}_{r, \rho, z} \stackrel{\sim}{\longrightarrow}\left(\kappa(z) \hat{\otimes}_{L} D_{r}\left(U_{z}^{(e)}\right)\right) / \mathfrak{b}_{\pi(z)}\left(\kappa(z) \hat{\otimes}_{L} D_{r}\left(U_{z}^{(e)}\right)\right)
$$




\section{From representations to sheaves}

In this section, as well as in Sections 10 and 11, we assume that

$$
L=\mathbb{Q}_{p} \quad \text { and } e>e_{\mathrm{st}} \quad \text { and } \quad r=r_{m}=\sqrt[p^{m}]{1 / p} \text { for some } m \geq 0 .
$$

Our proposed "localization functor" from representations to sheaves associated to the pair $\sigma(\chi)=\theta$ will be a functor

$$
\mathscr{L}_{r, \chi}: M \mapsto \mathscr{D}_{r, \chi} \otimes_{\underline{D}_{r, \theta}} \underline{M}_{r}
$$

from (coadmissible) left $D(G)_{\theta}$-modules $M$ to left $\mathscr{D}_{r, \chi}$-modules satisfying additional properties. Here $\underline{M}_{r}$ is a constructible sheaf replacing the constant sheaf $\underline{M}$ appearing in the Beilinson-Bernstein construction; see Theorem 5.2.2. It is a modest generalization of the sheaf $\underline{D}_{r}$ as follows.

8.1. A constructible sheaf of modules. Suppose we are given any (left) $D(G)$ module $M$. Let $F \subseteq \mathscr{B}$ be a facet. We may regard $M$ as a $D\left(U_{F}^{(e)}\right)$-module via the natural map $D\left(U_{F}^{(e)}\right) \rightarrow D(G)$. We put

$$
M_{r}\left(U_{F}^{(e)}\right):=D_{r}\left(U_{F}^{(e)}\right) \otimes_{D\left(U_{F}^{(e)}\right)} M,
$$

a (left) $D_{r}\left(U_{F}^{(e)}\right)$-module. If $F^{\prime} \subseteq \mathscr{B}$ is another facet such that $F^{\prime} \subset \bar{F}$ the map

$$
\sigma_{r}^{F^{\prime} F} \otimes \mathrm{id}: M_{r}\left(U_{F^{\prime}}^{(e)}\right) \rightarrow M_{r}\left(U_{F}^{(e)}\right), \quad \delta \otimes m \mapsto \sigma_{r}^{F^{\prime} F}(\delta) \otimes m
$$

is a module homomorphism relative to $\sigma_{r}^{F^{\prime} F}$ and inherits the homomorphic properties from $\sigma_{r}^{F^{\prime} F}$ (Lemma 4.3.5). Again, we may define a sheaf of $K$-vector spaces $\underline{M}_{r}$ on $\mathscr{B}$ in a completely analogous way as the sheaf $\underline{D}_{r}$ by replacing each $D_{r}\left(U_{F}^{(\bar{e})}\right)$ and each $D_{r}\left(U_{z}^{(e)}\right)$ by $M_{r}\left(U_{F}^{(e)}\right)$ and $M_{r}\left(U_{z}^{(e)}\right)$ respectively. In particular, $\underline{M}_{r}$ restricted to a facet $F$ is the constant sheaf with value $M_{r}\left(U_{F}^{(e)}\right)$ and therefore $\underline{M}_{r}$ is a constructible sheaf. If $s \in D_{r}\left(U_{z}^{(e)}\right), m \in M_{r}\left(U_{z}^{(e)}\right)$ the "pointwise multiplication" $(s \cdot m)(z):=s(z) m(z)$ makes $\underline{M}_{r}$ a $\underline{D}_{r}$-module.

Lemma 8.1.1. If $M$ is a $D(G)_{\theta}$-module then $\underline{M}_{r}$ is a $\underline{D}_{r, \theta}$-module via the morphism $\underline{D}_{r} \rightarrow \underline{D}_{r, \theta}$.

Proof. This is easy to see.

\subsection{A localization functor.}

8.2.1. As usual, $\mathscr{D}_{r, \chi} \otimes_{\underline{\underline{r}}_{r, \theta}} \underline{M}_{r}$ denotes the sheaf associated to the presheaf $V \mapsto$ $\mathscr{D}_{r, \chi}(V) \otimes_{\underline{D}_{r, \theta}(V)} \underline{M}_{r}(V)$ on $\mathscr{B}$. The construction $M \mapsto \underline{M}_{r}$ is functorial in $M$ and commutes with arbitrary direct sums. Thus the correspondence

$$
\mathscr{L}_{r, \chi}: M \mapsto \mathscr{D}_{r, \chi} \otimes_{\underline{D}_{r, \theta}} \underline{M}_{r}
$$


is a covariant functor from (left) $D(G)_{\theta}$-modules to (left) $\mathscr{D}_{r, \chi}$-modules. It commutes with arbitrary direct sums. We call it tentatively a localization functor associated to $\chi$.

We emphasize that the functor $\mathscr{L}_{r, \chi}$ depends on the choice of the level $e$. As we did before we suppress this dependence in the notation. As a second remark, let $\mathcal{M}$ be an arbitrary $\mathscr{D}_{r, \chi}$-module and $f: \mathscr{L}_{r, \chi}(M) \rightarrow \mathcal{M}$ a morphism. The composite

$$
M \rightarrow \Gamma\left(\mathscr{B}, \underline{M}_{r}\right) \rightarrow \Gamma\left(\mathscr{B}, \mathscr{L}_{r, \chi}(M)\right) \stackrel{f}{\rightarrow} \Gamma(\mathscr{B}, \mathcal{M})
$$

is a $K$-linear map. We therefore have a natural transformation of functors

$$
\operatorname{Hom}_{\mathscr{D}_{r, \chi}}\left(\mathscr{L}_{r, \chi}(\cdot), .\right) \rightarrow \operatorname{Hom}_{K}(\cdot, \Gamma(\mathscr{B}, \cdot)) \text {. }
$$

Generally, it is far from being an equivalence.

We compute the stalks of the localization $\mathscr{L}_{r, \chi}(M)$ for a coadmissible module $M$. In this case $\left(\underline{M}_{r}\right)_{z}$ is finitely generated over the Banach algebra $D_{r}\left(U_{z}^{(e)}\right)_{\theta}$ and therefore has a unique structure as a Banach module over $D_{r}\left(U_{z}^{(e)}\right)_{\theta}$. Let $z \in \mathscr{B} \subset X^{\text {an }}$ with residue field $\kappa(z)$. Recall that $\pi(z)$ equals the generic point of $X$.

Proposition 8.2.2. Let $M$ be a coadmissible left $D(G)_{\theta}$-module and let $z \in \mathscr{B}$. The morphism $\underline{M}_{r} \rightarrow \mathscr{L}_{r, \chi}(M)$ induces an isomorphism between the $\lambda$-coinvariants of the $\mathfrak{t}_{K}$-module

$$
\left(\kappa(z) \hat{\otimes}_{L}\left(\underline{M}_{r}\right)_{z}\right) / \mathfrak{n}_{\pi(z)}\left(\kappa(z) \hat{\otimes}_{L}\left(\underline{M}_{r}\right)_{z}\right)
$$

and the stalk $\mathscr{L}_{r, \chi}(M)_{z}$. In particular, if $\theta=\theta_{0}$ we have

$$
\left(\kappa(z) \hat{\otimes}_{L}\left(\underline{M}_{r}\right)_{z}\right) / \mathfrak{b}_{\pi(z)}\left(\kappa(z) \hat{\otimes}_{L}\left(\underline{M}_{r}\right)_{z}\right) \stackrel{\sim}{\longrightarrow} \mathscr{L}_{r, \rho}(M)_{z} .
$$

Proof. Let $N$ be an arbitrary finitely generated $D_{r}\left(U_{z}^{(e)}\right)$-module. According to Lemma 7.4.12 the space $\mathscr{D}_{r, \mathfrak{t}, z} \otimes_{D_{r}\left(U_{z}^{(e)}\right)} N$ may be written as

$$
\begin{array}{r}
{\left[\left(\left(\kappa(z) \hat{\otimes}_{L} D_{r}\left(U_{z}^{(e)}\right)\right) / \mathfrak{n}_{\pi(z)}(\kappa(z)) \hat{\otimes}_{L} D_{r}\left(U_{z}^{(e)}\right)\right) \otimes_{\kappa(z) \hat{\otimes}_{L} D_{r}\left(U_{z}^{(e)}\right)} \kappa(z) \hat{\otimes}_{L} D_{r}\left(U_{z}^{(e)}\right)\right]} \\
\otimes_{D_{r}\left(U_{z}^{(e)}\right)} N .
\end{array}
$$

Since $N$ is a complete Banach module this may be identified with

$$
\left(\kappa(z) \hat{\otimes}_{L} N\right) / \mathfrak{n}_{\pi(z)}\left(\kappa(z) \hat{\otimes}_{L} N\right)
$$

by associativity of the completed tensor product. The resulting isomorphism

$$
\left(\kappa(z) \hat{\otimes}_{L} N\right) / \mathfrak{n}_{\pi(z)}\left(\kappa(z) \hat{\otimes}_{L} N\right) \stackrel{\sim}{\longrightarrow} \mathscr{D}_{r, \mathfrak{t}, z} \otimes_{D_{r}\left(U_{z}^{(e)}\right)} N
$$

is functorial in $N$. According to the second part of loc.cit. we obtain a functorial homomorphism

$\left(\lambda\right.$-coinvariants of $\left.\left(\kappa(z) \hat{\otimes}_{L} N\right) / \mathfrak{n}_{\pi(z)}\left(\kappa(z) \hat{\otimes}_{L} N\right)\right) \rightarrow \mathscr{D}_{r, \chi, z} \otimes_{D_{r}\left(U_{z}^{(e)}\right)} N$, 
which is an isomorphism in the case $N=D_{r}\left(U_{z}^{(e)}\right)$. Note that the target is a right exact functor in $N$. Similarly, the source is also a right exact functor in $N$. To see this, it suffices to note that the functor which sends $N$ to $\kappa(z) \hat{\otimes}_{L} N$ is exact. Indeed, any short exact sequence of finitely generated $D_{r}\left(U_{z}^{(e)}\right)$-modules is a strict exact sequence relative to the unique Banach topology on such modules (cf. [Schneider and Teitelbaum 2003, Proposition 2.1.iii]) and so the claim follows from a wellknown result of L. Gruson [1966, 3.2, Corollaire 1]. Since the source and the target are both right exact functors in $N$ commuting with finite direct sums, we may use a finite free presentation of $N$ to obtain that it is an isomorphism in general. The assertion of the proposition follows by taking $N=\left(\underline{M}_{r}\right)_{z}$.

Corollary 8.2.3. Let $\chi$ be dominant and regular. The functor $\mathscr{L}_{r, \chi}$, restricted to coadmissible modules, is exact.

Proof. Exactness can be checked at a point $z \in \mathscr{B}$, where the functor in question equals the composite of three functors. The first functor equals

$$
N \mapsto D_{r}\left(U_{z}^{(e)}\right)_{\theta} \otimes_{D\left(U_{z}^{(e)}\right)_{\theta}} N
$$

on the category of coadmissible $D\left(U_{z}^{(e)}\right)_{\theta}$-modules. It is exact by [Schneider and Teitelbaum 2003, Remark 3.2]. The second functor equals $N \mapsto \kappa(z) \hat{\otimes}_{L} N$ on the category of finitely generated $D_{r}\left(U_{z}^{(e)}\right)_{\theta}$-modules. It is exact as we have explained in the proof of the preceding proposition. The natural inclusion $U(\mathfrak{g})_{\theta} \rightarrow D_{r}\left(U_{z}^{(e)}\right)_{\theta}$ allows one to consider $\kappa(z) \hat{\otimes}_{L} N$ as a $U\left(\kappa(z) \otimes_{L} \mathfrak{g}\right)_{\theta}$-module. The BeilinsonBernstein stalk functor at $\pi(z)$ of the corresponding localization on the flag variety $X_{\kappa(z)}$ (note that the natural embedding $k(\pi(z)) \rightarrow \kappa(z)$ gives a canonical lift of $\pi(z)$ to a $\kappa(z)$-rational point of $\left.X_{\kappa(z)}\right)$ is given by the $\lambda$-coinvariants of the $\kappa(z) \otimes_{L} \mathfrak{h}$ module

$$
\left(\kappa(z) \hat{\otimes}_{L} N\right) / \mathfrak{n}_{\pi(z)}\left(\kappa(z) \hat{\otimes}_{L} N\right)
$$

according to Theorem 5.2.2(iii). By part (ii) of the same theorem, this functor is exact if $\chi$ is dominant and regular.

Lemma 8.2.4. Let $z \in \mathscr{B}$. If $N$ is a finitely generated $D_{r}\left(U_{z}^{(e)}\right)_{\theta}$-module which is finite dimensional over $K$, then the natural map

$$
\mathcal{D}_{\mathscr{B}, \chi, z}^{\text {an }} \otimes_{U\left(\mathfrak{g}_{K}\right)_{\theta}} N \stackrel{\sim}{\longrightarrow} \mathscr{D}_{r, \chi, z} \otimes_{D_{r}\left(U_{z}^{(e)}\right)_{\theta}} N
$$

is an isomorphism which is functorial in modules of this kind.

Proof. We adopt the notation of Proposition 6.4.8 and write

$$
\underset{V}{\lim }\left(\mathcal{A}_{V} \# D_{r}\left(U_{z}^{(e)}\right)\right) \stackrel{\sim}{\longrightarrow} \mathcal{O}_{\mathscr{B}, z} \# D_{r}\left(U_{z}^{(e)}\right),
$$

an isomorphism of $K$-algebras according to Proposition 2.4.1. By Proposition 2.1 
of [Schneider and Teitelbaum 2003] the finitely generated module

$$
\left(\mathcal{A}_{V} \# D_{r}\left(U_{z}^{(e)}\right)\right) \otimes_{D_{r}\left(U_{z}^{(e)}\right)} N
$$

has a unique Banach topology. We thus have canonical $\mathcal{A}_{V}$-linear isomorphisms

$$
\left(\mathcal{A}_{V} \hat{\otimes}_{L} D_{r}\left(U_{z}^{(e)}\right)\right) \otimes_{D_{r}\left(U_{z}^{(e)}\right)} N \simeq \mathcal{A}_{V} \hat{\otimes}_{L} N=\mathcal{A}_{V} \otimes_{L} N .
$$

Passage to the inductive limit yields, by Proposition 2.4.1, the $\mathcal{O}_{\mathscr{B}, z}$-linear map

$$
\left(\mathcal{O}_{\mathscr{B}, z} \hat{\otimes} D_{r}\left(U_{z}^{(e)}\right)\right) \otimes_{D_{r}\left(U_{z}^{(e)}\right)} N \simeq \mathcal{O}_{\mathscr{B}, z} \otimes_{L} N=\left(\mathcal{O}_{\mathscr{B}, z} \# U\left(\mathfrak{g}_{K}\right)\right) \otimes_{U\left(\mathfrak{g}_{K}\right)} N .
$$

The target maps canonically to $\mathcal{D}_{\mathscr{B}, \chi, z}^{\text {an }} \otimes_{U\left(\mathfrak{g}_{K}\right)_{\theta}} N$ and the composed map annihilates all elements of the form $\xi \hat{\otimes} n$ with $n \in N$ and $\xi \in \mathcal{I}_{\mathscr{B}, \chi, z}^{\text {an }}$. Since such $\xi$ generate $\mathscr{I}_{\chi, z}^{\text {an }}$ the composed map factors therefore into a map

$$
\mathscr{D}_{r, \chi, z} \otimes_{D_{r}\left(U_{z}^{(e)}\right)_{\theta}} N \rightarrow \mathcal{D}_{\mathscr{B}, \chi, z}^{\mathrm{an}} \otimes_{U\left(\mathfrak{g}_{K}\right)_{\theta}} N
$$

This gives the required inverse map.

Corollary 8.2.5. Let $M$ be a left $D(G)_{\theta}$-module such that $\operatorname{dim}_{K} M_{r}\left(U_{z}^{(e)}\right)<\infty$ for all $z \in \mathscr{B}$. The natural morphism of sheaves

$$
\mathcal{D}_{\mathscr{B}, \chi}^{\mathrm{an}} \otimes_{U\left(\mathfrak{g}_{K}\right)_{\theta}} \underline{M}_{r} \stackrel{\sim}{\longrightarrow} \mathscr{D}_{r, \chi} \otimes_{\underline{D}_{r, \theta}} \underline{M}_{r}=\mathscr{L}_{r, \chi}(M)
$$

induced from (7.4.8) is an isomorphism.

Proof. Let $z \in \mathscr{B}$. Applying the preceding lemma to $N:=M_{r}\left(U_{z}^{(e)}\right)$ we see that the morphism is an isomorphism at the point $z$. This proves the claim.

\subsection{Equivariance.}

8.3.1. Consider for a moment an arbitrary ringed space $(Y, \mathcal{A})$, where $\mathcal{A}$ is a sheaf of (not necessarily commutative) $K$-algebras on $Y$. Let $\Gamma$ be an abstract group acting (from the right) on $(Y, \mathcal{A})$. In other words, for every $g, h \in \Gamma$ and every open subset $U \subseteq Y$ there is an isomorphism of $K$-algebras $g^{*}: \mathcal{A}(U) \stackrel{\sim}{\longrightarrow} \mathcal{A}\left(g^{-1} U\right)$ compatible in an obvious sense with restriction maps and satisfying $(g h)^{*}=h^{*} g^{*}$.

A $\Gamma$-equivariant $\mathcal{A}$-module (see [Jantzen 2003, II.F.5]) is a (left) $\mathcal{A}$-module $\mathcal{M}$ equipped, for any open subset $U \subseteq Y$ and for $g \in G$, with $K$-linear isomorphisms $g^{*}: \mathcal{M}(U) \stackrel{\sim}{\longrightarrow} \mathcal{M}\left(g^{-1} U\right)$ compatible with restriction maps and such that $g^{*}(a m)=$ $g^{*}(a) g^{*}(m)$ for $a \in \mathcal{A}(U), m \in \mathcal{M}(U)$. If $g, h \in G$ we require $(g h)^{*}=h^{*} g^{*}$.

An obvious example is $\mathcal{M}=\mathcal{A}$. If $\mathcal{M}$ is equivariant we have a $K$-linear isomorphism $\mathcal{M}_{z} \stackrel{\sim}{\longrightarrow} \mathcal{M}_{g^{-1} z}$ between the stalks of the sheaf $\mathcal{M}$ at $z$ and $g^{-1} z$ for any $g \in G$. Finally, a morphism of equivariant modules is an $\mathcal{A}$-linear map compatible with the $\Gamma$-actions. The equivariant modules form an abelian category. 
8.3.2. After these preliminaries we go back to the situation discussed in the previous section. We keep all the assumptions from this section. The group $G$ naturally acts on the ringed space $\left(X^{\text {an }}, \mathcal{O}_{X}\right.$ an $)$. Moreover, $G$ acts on $\mathfrak{g}$ and $U(\mathfrak{g})$ via the adjoint action as usual. It follows from the classical argument [Miličić 1993a, Section 3] that the sheaves

$$
\mathcal{O}_{X} \text { an } \# U(\mathfrak{g}), \mathcal{I}_{\chi}^{\text {an }} \quad \text { and } \quad \mathcal{D}_{\chi}^{\text {an }}:=\left(\mathcal{O}_{X} \text { an } \# U(\mathfrak{g})\right) / \mathcal{I}_{\chi}^{\text {an }}
$$

(as defined in Section 6) are equivariant $\mathcal{O}_{X}$ an-modules. Of course, here

$$
g^{*}: \mathcal{D}_{\chi}^{\mathrm{an}}(U) \stackrel{\sim}{\longrightarrow} \mathcal{D}_{\chi}^{\mathrm{an}}\left(g^{-1} U\right)
$$

is even a $K$-algebra isomorphism for all $g \in G$ and open subsets $U \subseteq X^{\text {an }}$.

On the other hand, the group $G$ acts on the ringed space $\left(\mathscr{B}, \mathcal{O}_{\mathscr{B}}\right)$ and the natural map $\vartheta_{\boldsymbol{B}}: \mathscr{B} \rightarrow X^{\text {an }}$ is $G$-equivariant, see Theorem 6.2.1. Since our functor $\vartheta_{\boldsymbol{B}}^{-1}$ preserves $G$-equivariance the $\mathcal{O}_{\mathscr{B}}$-modules

$$
\mathcal{O}_{\mathscr{B}} \# U\left(\mathfrak{g}_{K}\right), \mathcal{I}_{\mathscr{B}, \chi}^{\text {an }} \quad \text { and } \quad \mathcal{D}_{\mathscr{B}, \chi}^{\text {an }}=\left(\mathcal{O}_{\mathscr{B}} \# U\left(\mathfrak{g}_{K}\right)\right) / \mathcal{I}_{\mathscr{B}, \chi}^{\text {an }}
$$

are $G$-equivariant. Again, here $g^{*}: \mathcal{D}_{\mathscr{B}, \chi}^{\text {an }}(U) \stackrel{\sim}{\longrightarrow} \mathcal{D}_{\mathscr{B}, \chi}^{\text {an }}\left(g^{-1} U\right)$ is a $K$-algebra isomorphism for all $g \in G$ and open subsets $U \subseteq \mathscr{B}$. Recall from Definition 7.2.1 the sheaf of $K$-algebras $\mathcal{O}_{\mathscr{B}} \# \underline{D}_{r}$.

Proposition 8.3.3. The $\mathcal{O}_{\mathscr{B}}$-module $\mathcal{O}_{\mathscr{B}} \# \underline{D}_{r}$ is $G$-equivariant. For $g \in G$ the map $g^{*}$ is a K-algebra isomorphism.

Proof. Given $g \in G$ and $z \in \mathscr{B}$ we have the group isomorphism

$$
g^{-1}(\cdot) g: U_{z}^{(e)} \stackrel{\sim}{\longrightarrow} U_{g^{-1} z}^{(e)}
$$

by (4.1.6). Since it is compatible with variation of the level $e$ it is compatible with the $p$-valuations $\stackrel{\circ}{\omega}_{z}$ and $\stackrel{\circ}{\omega}_{g}{ }^{-1} z$. It induces therefore an isometric isomorphism of Banach algebras

$$
g^{-1}(\cdot) g: D_{r}\left(U_{z}^{(e)}\right) \stackrel{\sim}{\longrightarrow} D_{r}\left(U_{g^{-1} z}^{(e)}\right) .
$$

The induced map

$$
\mathcal{O}_{\mathscr{B}, z} \hat{\otimes}_{L} D_{r}\left(U_{z}^{(e)}\right) \stackrel{\sim}{\longrightarrow} \mathcal{O}_{\mathscr{B}, z} \hat{\otimes}_{L} D_{r}\left(U_{g^{-1} z}^{(e)}\right)
$$

is multiplicative with respect to the skew multiplication and we obtain an isomorphism of $K$-algebras

$$
g^{*}:\left(\mathcal{O}_{\mathscr{B}} \# \underline{D}_{r}\right)_{z} \stackrel{\sim}{\longrightarrow}\left(\mathcal{O}_{\mathscr{B}} \# \underline{D}_{r}\right)_{g^{-1} z}
$$

according to Lemma 7.2.3. Since we have the identity $g \mathfrak{x} g^{-1}=\operatorname{Ad}(g)(\mathfrak{x})$ in $D(G)$ 
this isomorphism fits into the commutative diagram

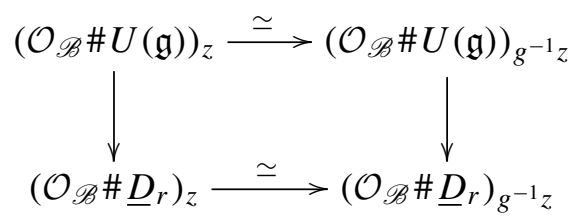

where the vertical arrows are the inclusions from (6.4.12). Recall the sheaf $\mathcal{F}$ appearing in Lemma 7.2.2. Let $\Omega \subseteq \mathscr{B}$ be an open subset. The isomorphisms (8.3.5) for $z \in \Omega$ assemble to a $K$-algebra isomorphism

$$
g^{*}: \mathcal{F}(\Omega) \stackrel{\sim}{\longrightarrow} \mathcal{F}\left(g^{-1} \Omega\right), s \mapsto\left[z \mapsto\left(g^{*}\right)^{-1}(s(g z))\right]
$$

compatible with restriction maps and satisfying $(g h)^{*}=h^{*} g^{*}$ for $g, h \in G$. It now suffices to see that $g^{*}$ maps the subspace $\left(\mathcal{O}_{\mathscr{B}} \# \underline{D}_{r}\right)(\Omega)$ into $\left(\mathcal{O}_{\mathscr{B}} \# \underline{D}_{r}\right)\left(g^{-1} \Omega\right)$. Let $s \in\left(\mathcal{O}_{\mathscr{B}} \# \underline{D}_{r}\right)(\Omega)$. If $F$ is a facet in $\mathscr{B}$ we let $\Omega=\bigcup_{i \in I} \Omega_{i}$ be a datum for $s$ with respect to $F$. If $F \cap \Omega_{i} \neq \varnothing$ we consider $g^{-1} V_{i}$ and $\left(g^{*}\right)^{-1}\left(s_{i}\right)$ and obtain a datum $g^{-1} \Omega=\bigcup_{i \in I} g^{-1} \Omega_{i}$ for the section $\left(g^{*}\right)^{-1} s g \in \mathcal{F}\left(g^{-1} \Omega\right)$ with respect to the facet $g^{-1} F$. Indeed, the axiom (a) of Definition 7.2.1 for the section $\left(g^{*}\right)^{-1} s g$ follows from the commutativity of the diagram

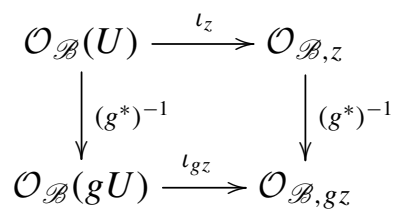

valid for any open subset $U \subseteq \mathscr{B}$ containing $z$. Moreover, we have a commutative diagram

$$
\begin{gathered}
D_{r}\left(U_{F^{\prime}}^{(e)}\right) \stackrel{\sigma_{r}^{F^{\prime} F}}{\longrightarrow} D_{r}\left(U_{F}^{(e)}\right) \\
\quad \downarrow\left(g^{*}\right)^{-1} \underset{g^{*}{ }^{*-1}}{ } \\
D_{r}\left(U_{g F^{\prime}}^{(e)} \stackrel{\sigma_{r}^{g F^{\prime}} g F}{\longrightarrow} D_{r}\left(U_{g F}^{(e)}\right)\right.
\end{gathered}
$$

whenever $F^{\prime}, F$ are two facets in $\mathscr{B}$ with $F^{\prime} \subseteq \bar{F}$. From this the axiom (b) for the section $\left(g^{*}\right)^{-1} s g$ follows easily.

It follows from the preceding proof that the morphism $\mathcal{O}_{\mathscr{B}} \# U(\mathfrak{g}) \rightarrow \mathcal{O}_{\mathscr{B}} \# \underline{D}_{r}$ from Proposition 7.2.6 is equivariant. The equivariant structure of $\mathcal{I}_{\mathscr{B}, \chi}^{\text {an }}$ therefore implies that the ideal sheaf $\mathscr{I}_{\chi}^{\text {an }}$ of $\mathcal{O}_{\mathscr{B}} \# \underline{D}_{r}$ is naturally equivariant. This yields the following corollary.

Corollary 8.3.6. The $\mathcal{O}_{\mathscr{B}}$-module $\mathscr{D}_{r, \chi}$ is equivariant. The map $g^{*}$ is a $K$-algebra isomorphism for any $g \in G$. The morphism $\mathcal{D}_{\mathscr{B}, \chi}^{\mathrm{an}} \rightarrow \mathscr{D}_{r, \chi}$ from (7.4.8) is equivariant. 
The above discussion shows that there is a natural right action of $G$ on the ringed space $\left(\mathscr{B}, \mathscr{D}_{r, \chi}\right)$. We let $\operatorname{Mod}_{G}\left(\mathscr{D}_{r, \chi}\right)$ be the abelian category of $G$-equivariant (left) $\mathscr{D}_{r, \chi}$-modules.

8.3.7. Using very similar arguments we may use the isomorphisms (8.3.4) appearing in the above proof to define an equivariant structure on the sheaves $\underline{D}_{r}$ and $\underline{D}_{r, \theta}$. As before we suppose $\sigma(\chi)=\theta$. If $M$ is a $D(G)$-module (resp. $D(G)_{\theta}$-module) with $m \in M$ and $g \in G$ we put $g . m:=\delta_{g^{-1}} m$. This defines a $K$-linear isomorphism

$$
g^{*}: M_{r}\left(U_{z}^{(e)}\right) \stackrel{\sim}{\longrightarrow} M_{r}\left(U_{g^{-1} z}^{(e)}\right)
$$

via $g^{*}(\delta \otimes m):=g^{*}(\delta) \otimes g m$ for any $\delta \in D_{r}\left(U_{z}^{(e)}\right)$. As in the case of $\underline{D}_{r}$ these isomorphisms lift to an equivariant structure on the sheaf $\underline{M}_{r}$. Since these isomorphisms are compatible with the isomorphisms (8.3.4) we obtain that $\underline{M}_{r}$ is an equivariant $\underline{D}_{r}$-module (resp. $\underline{D}_{r, \theta}$-module). We now define $g^{*}(\partial \otimes m):=g^{*}(\partial) \otimes g^{*}(m)$ for local sections $\partial$ and $m$ of $\mathscr{D}_{r, \chi}$ and $\underline{M}_{r}$ respectively. Since the morphism $\underline{D}_{r, \theta} \rightarrow \mathscr{D}_{r, \chi}$ induced by Proposition 7.2.5 is equivariant this yields an equivariant structure on $\mathscr{L}_{r, \chi}(M)$. If $M \rightarrow N$ is a $D(G)_{\theta}$-linear map the resulting morphism $\mathscr{L}_{r, \chi}(M) \rightarrow \mathscr{L}_{r, \chi}(N)$ is easily seen to be equivariant. This shows

Corollary 8.3.8. The functor $\mathscr{L}_{r, \chi}$ takes values in $\operatorname{Mod}_{G}\left(\mathscr{D}_{r, \chi}\right)$.

\section{Comparison with the Schneider-Stuhler construction}

In this section we assume $L=\mathbb{Q}_{p}, e>e_{\mathrm{st}}, e_{c l}$ and $r \in\left[r_{0}, 1\right)$. We will work in this section with the trivial infinitesimal character, i.e., $\lambda:=\lambda_{0}$ and $\theta:=\theta_{0}$.

\subsection{Preliminaries on smooth distributions.}

9.1.1. Let $M$ be a co-admissible $D(G)$-module such that the associated locally analytic representation $V=M_{b}^{\prime}$ is smooth. In the previous section, we have associated to $M$ a sheaf $\underline{M}_{r}$ on the Bruhat-Tits building $\mathscr{B}$. On the other hand, we also have the sheaf $\underset{\approx}{V}$ on $\mathscr{B}$ constructed in [Schneider and Stuhler 1997, 4.6]. We now show that for $\underset{r}{\approx}<p^{-1 /(p-1)}$, the two sheaves $\underset{\approx}{\check{V}}$ and $\underline{M}_{r}$ are canonically isomorphic. Here, $\check{V}$ denotes the smooth dual. We remark straightaway that $V$ is admissible-smooth [Schneider and Teitelbaum 2003, Theorem 6.6] and hence, so is $\check{V}$ [Cartier 1979, 1.5(c)].

Suppose $H$ is a uniform locally $\mathbb{Q}_{p}$-analytic group with $\mathbb{Q}_{p}$-Lie algebra $\mathfrak{h}$. Let $D^{\infty}(H)$ denote the quotient of $D(H)$ by the ideal generated by $\mathfrak{h}$. Let $\mathcal{C}_{H}^{\infty}$ denote the category of coadmissible $D^{\infty}(H)$-modules. If $U_{r}(\mathfrak{h})$ denotes the closure of $U(\mathfrak{h})$ inside $D_{r}(H)$ we put

$$
H_{(r)}:=H \cap U_{r}(\mathfrak{h})
$$


Lemma 9.1.2. The set $H_{(r)}$ is an open normal subgroup of $H$ constituting, for $r \uparrow 1$, a neighborhood basis of $1 \in H$.

Proof. As the norm $\|\cdot\|_{r}$ on $D_{r}(H)$ does not depend on the choice of ordered basis the inversion map $h \mapsto h^{-1}$ induces an automorphism of $D_{r}(H)$. It induces an automorphism of $U_{r}(\mathfrak{h})$, which implies that $H_{(r)}$ is a subgroup of $H$. A similar argument with the conjugation automorphism $h \mapsto g h g^{-1}$ for a $g \in H$ implies that this subgroup is normal in $H$. For the remaining assertions we choose $m \geq 0$ such that $r_{m}=\sqrt[p m]{r_{0}} \geq r$ and consider $D\left(P_{m+1}(H)\right)$. The inclusion $D\left(P_{m+1}(H)\right) \subseteq D(H)$ gives rise to an isometric embedding

$$
D_{r_{0}}\left(P_{m+1}(H)\right) \hookrightarrow D_{r_{m}}(H)
$$

(final remark in Section 2.2.3). Since $U(\mathfrak{h})$ is norm-dense inside $D_{r_{0}}\left(P_{m+1}(H)\right)$ it follows that $P_{m+1}(H) \subset U_{r_{m}}(\mathfrak{h}) \subseteq U_{r}(\mathfrak{h})$, which implies $P_{m+1}(H) \subseteq H_{(r)}$ and therefore $H_{(r)}$ is open. Finally, if $r \uparrow 1$ then $r_{m} \uparrow 1$ whence $m \uparrow \infty$. Since the lower $p$-series $\left\{P_{m}(H)\right\}_{m}$ constitutes a neighborhood basis of $1 \in H$ the last assertion of the lemma follows.

By the proof of Theorem 6.6 in [Schneider and Teitelbaum 2003], the lemma implies a canonical $K$-algebra isomorphism $D^{\infty}(H) \simeq \lim _{r} K\left[H / H_{(r)}\right]$ coming from restricting distributions to the subspace of $K$-valued locally constant functions on $H$.

Proposition 9.1.3. (i) We have $D_{r}(H) \otimes_{D(H)} D^{\infty}(H) \simeq K\left[H / H_{(r)}\right]$ as right $D^{\infty}(H)$-modules.

(ii) If $M \in \mathcal{C}_{H}^{\infty}$ and $V=M_{b}^{\prime}$ denotes the corresponding smooth representation then $D_{r}(H) \otimes_{D(H)} M \simeq(\check{V})_{H_{(r)}}$ as $K$-vector spaces. Here, $(\cdot)_{H_{(r)}}$ denotes $H_{(r)^{-}}$ coinvariants and $(\cdot)$ denotes the smooth dual.

Proof. The first statement follows from $D_{r}(H)=\bigoplus_{h \in H / H_{(r)}} \delta_{h} U_{r}(\mathfrak{h})$ as right $U_{r}(\mathfrak{h})$ modules by passing to quotients modulo the ideals generated by $\mathfrak{h}$. The second statement follows from (i) by observing the general identities $K[H / N] \otimes_{D^{\infty}(H)} M=$ $\operatorname{Hom}_{K}\left(V^{N}, K\right)=(\check{V})_{N}$ valid for any normal open subgroup $N$ of $H$.

Corollary 9.1.4. If $M \in \mathcal{C}_{H}^{\infty}$ and $r_{0} \leq r<p^{-1 / p-1}$ then $D_{r}(H) \otimes_{D(H)} M \simeq(\check{V})_{H}$. Proof. We have $U_{r}(\mathfrak{h})=D_{r}(H)$ for such an $r$ and therefore $H_{(r)}=H$.

\subsection{The comparison isomorphism.}

9.2.1. Let us return to our sheaf $M \mapsto \underline{M}_{r}$. We assume in the following that

$$
r_{0} \leq r<p^{-1 / p-1} \text {. }
$$


Let $F$ be a facet in $X$. If we apply the above corollary to the uniform group $U_{F}^{(e)}$ we obtain a canonical linear isomorphism

$$
f_{r}^{F}: M\left(U_{F}^{(e)}\right)=D_{r}\left(U_{F}^{(e)}\right) \otimes_{D\left(U_{F}^{(e)}\right)} M \stackrel{\sim}{\longrightarrow}(\check{V})_{U_{F}^{(e)}} .
$$

If $F \subseteq \overline{F^{\prime}}$ for two facets $F, F^{\prime}$ in $X$ it follows that

$$
f_{r}^{F^{\prime}} \circ \sigma_{r}^{F F^{\prime}}=\operatorname{pr}^{F F^{\prime}} \circ f_{r}^{F}
$$

where $\operatorname{pr}^{F F^{\prime}}:(\check{V})_{U_{F}^{(e)}} \rightarrow(\check{V})_{U_{F^{\prime}}^{(e)}}$ denotes the natural projection.

Proposition 9.2.3. Given an open subset $\Omega \subseteq X$, the collection of maps $f_{r}^{z}$ for $z \in \Omega$ induces a $K$-linear isomorphism $\underline{M}_{r}(\Omega) \simeq \underset{\approx}{\check{V}}(\Omega)$ compatible with restriction maps whence a canonical isomorphism of sheaves

$$
\underline{M}_{r} \stackrel{\sim}{\longrightarrow} \underset{\sim}{\check{V}}
$$

which is natural in admissible $V$.

Proof. Given $z \in \mathscr{B}$ we have the isomorphism

$$
f_{r}^{z}: M_{r}\left(U_{z}^{(e)}\right) \stackrel{\sim}{\longrightarrow}(\check{V})_{U_{z}^{(e)}},
$$

as explained above. These maps assemble to a $K$-linear isomorphism, say $f_{r}^{\Omega}$, between the space of maps

$$
s: \Omega \rightarrow \bigcup_{z \in \Omega} M_{r}\left(U_{z}^{(e)}\right)
$$

such that $s(z) \in M_{r}\left(U_{z}^{(e)}\right)$ for all $z \in \mathscr{B}$ and the space of maps

$$
s: \Omega \rightarrow \bigcup_{z \in \Omega}(\check{V})_{U_{z}^{(e)}}
$$

such that $s(z) \in(\check{V})_{U_{z}^{(e)}}$ for all $z \in \mathscr{B}$. It is clearly compatible with restriction. It therefore suffices to show that it descends to an isomorphism between the subspaces $\underline{M}_{r}(\Omega)$ and $\underset{\approx}{\check{V}}(\Omega)$ respectively. Since $\underline{M}_{r}$ and $\underset{\widetilde{V}}{\check{V}}$ are sheaves it suffices to verify this over the open sets $\Omega \cap \operatorname{St}(F)$ for facets $F \subset \mathscr{B}$. We may therefore fix a facet $F \subset \mathscr{B}$ and assume that $\Omega \subseteq \operatorname{St}(F)$. Restricting to members $\Omega_{i}$ with $\Omega_{i} \cap F \neq \varnothing$ of a datum for $s$ with respect to $F$ and using the sheaf property a second time we may assume that the covering $\{\Omega\}$ of $\Omega=\Omega \cap \operatorname{St}(F)$ is a datum for $s$ with respect to $F$ satisfying $\Omega \cap F \neq \varnothing$. Let $s \in M_{r}\left(U_{F}^{(e)}\right)$ be the corresponding element of the datum. We let $\check{v}$ be any preimage in $\check{V}$ of $f_{r}^{F}(s) \in(\check{V})_{U_{F}^{(e)}}$. The value of the function $f_{r}^{\Omega}(s)$ at $z \in \Omega$ is then given by

$$
f_{r}^{z}(s(z))=f_{r}^{F^{\prime}}\left(\sigma_{r}^{F F^{\prime}}(s)\right) \stackrel{(9.2 .2)}{=} \operatorname{pr}^{F F^{\prime}}\left(f_{r}^{F}(s)\right)=\text { class of } \check{v} \in(\check{V})_{U_{F^{\prime}}^{(e)}},
$$

where $F^{\prime} \in \operatorname{St}(F)$ is the unique open facet containing $z$. This means $f_{r}^{\Omega}(s) \in \underset{\approx}{\check{V}}(\Omega)$. 
Conversely, let $\check{s} \in \underset{\sim}{\check{V}}(\Omega)$ and consider $s:=\left(f_{r}^{\Omega}\right)^{-1}(\check{s})$. Let $F \subset \mathscr{B}$ be a facet. Any defining open covering $\Omega=\bigcup_{i \in I} \Omega_{i}$ with vectors $\check{v}_{i} \in \check{V}$ for the section $\check{s}$ induces an open covering $\Omega \cap \operatorname{St}(F)=\bigcup_{i \in I} \Omega_{i, F}$, where $\Omega_{i, F}:=\Omega_{i} \cap \operatorname{St}(F)$. If $F \cap \Omega_{i, F} \neq \varnothing$ we let $s_{i} \in M_{r}\left(U_{F}^{(e)}\right)$ be the inverse image of the class of $\check{v}_{i}$ under $\left(f_{r}^{F}\right)^{-1}$. We claim that this gives a datum for $s$ with respect to $F$. Indeed, for any $z \in \Omega_{i, F} \cap F$ we compute

$$
s(z)=\left(f_{r}^{z}\right)^{-1}(\check{s}(z))=\left(f_{r}^{z}\right)^{-1}\left(\text { class of } \check{v}_{i}\right)=s_{i},
$$

which settles the axiom (a) for $s$. Similarly, for any $z^{\prime} \in \Omega_{i, F}$ the value of $s\left(z^{\prime}\right)$ equals

$$
\begin{aligned}
\left(f_{r}^{z^{\prime}}\right)^{-1}\left(\check{s}\left(z^{\prime}\right)\right) & =\left(f_{r}^{F^{\prime}}\right)^{-1}\left(\operatorname{class} \text { of } \check{v}_{i}\right) \\
& =\left(f_{r}^{F^{\prime}}\right)^{-1}\left(\operatorname{pr}^{F F^{\prime}}\left(\check{v}_{i}\right)\right) \stackrel{(9.2 .2)}{=} \sigma_{r}^{F F^{\prime}}\left(\left(f_{r}^{F}\right)^{-1}\left(\check{v}_{i}\right)\right)=\sigma_{r}^{F F^{\prime}}\left(s_{i}\right)
\end{aligned}
$$

where $F^{\prime}$ denotes the unique open facet of $\operatorname{St}(F)$ containing $z^{\prime}$. This proves (b) for $s$. All in all $s \in \underline{M}_{r}(\Omega)$. This proves the proposition.

Lemma 9.2.4. Let $M$ be a coadmissible $D^{\infty}(G)$-module. Then $M$ is a $D(G)_{\theta_{0}}{ }^{-}$ module.

Proof. We have to show that the canonical map $D(G) \rightarrow D^{\infty}(G)$ factors through $D(G)_{\theta_{0}}$. The kernel of $D(G) \rightarrow D^{\infty}(G)$ is the two sided ideal generated by $\mathfrak{g}$. The intersection of this latter ideal with $Z\left(\mathfrak{g}_{K}\right)$ equals $\operatorname{ker} \theta_{0}$ (see the example on page 1411). It follows that the map $Z\left(\mathfrak{g}_{K}\right) \rightarrow D^{\infty}(G)$ factors through $\theta_{0}$.

Theorem 9.2.5. Let $r=r_{0}$. Suppose $M$ is a coadmissible $D^{\infty}(G)$-module. Then there is a canonical isomorphism of $\mathcal{O}_{\mathscr{B}}$-modules

$$
C^{S S}: \mathcal{O}_{\mathscr{B}} \otimes_{L} \underset{\sim}{\stackrel{\check{V}}{\longrightarrow}} \stackrel{\sim}{\longrightarrow} \mathscr{L}_{r_{0}, \rho}(M)
$$

which is natural in such $M$. Here, as above, $V=M_{b}^{\prime}$.

Proof. Since $\mathfrak{g} M=0$ there is a canonical isomorphism

$$
\mathcal{O}_{\mathscr{B}} \otimes_{L} \underline{M}_{r_{0}} \stackrel{\sim}{\rightarrow} \mathcal{D}_{\mathscr{B} X}^{\text {an }} \otimes_{U\left(\mathfrak{g}_{K}\right)_{\theta}} \underline{M}_{r_{0}} .
$$

Arguing stalkwise the assertion follows from Corollary 8.2.5 and Proposition 9.2.3.

\section{Compatibility with the Beilinson-Bernstein localization}

Throughout this section we suppose that the conditions (8.0.1) are fulfilled.

Let $V$ denote a finite dimensional algebraic representation of $\boldsymbol{G}$. Then $V$ gives rise to a $U(\mathfrak{g})$-module. Let $M=V^{\prime}$ denote the dual of $V$. It is a coadmissible $D(G)$-module. Suppose the $U\left(\mathfrak{g}_{K}\right)$-module underlying $M$ is a $U\left(\mathfrak{g}_{K}\right)_{\theta}$-module. 
Recall that to any $U\left(\mathfrak{g}_{K}\right)_{\theta}$-module $M$, Beilinson and Bernstein associate a $\mathcal{D}_{\chi}$ module which will be denoted $\Delta(M)$ (see Section 5). We can pull this back under the natural map $\pi: X^{\text {an }} \rightarrow X$ to get a $\mathcal{D}_{\chi}^{\text {an }}$-module $\Delta(M)^{\text {an }}$. Finally, we may apply the functor $\vartheta_{\boldsymbol{B}}^{-1}$ to this module. Denote the latter $\mathcal{O}_{\mathscr{B}}$-module by $\Delta(M)_{\mathscr{B}}^{\mathrm{an}}$. One has the following description of $\Delta(M)^{\text {an }}$ and $\Delta(M)_{\mathscr{B}}^{\text {an }}$ :

$$
\begin{aligned}
\Delta(M)^{\mathrm{an}} & =\mathcal{D}_{\chi}^{\mathrm{an}} \otimes_{U\left(\mathfrak{g}_{K}\right)_{\theta}} M, \\
\Delta(M)_{\mathscr{B}}^{\mathrm{an}}=\mathcal{D}_{\mathscr{B}, \chi}^{\mathrm{an}} \otimes_{U\left(\mathfrak{g}_{K}\right)_{\theta}} M . &
\end{aligned}
$$

The second identity follows from the compatibility between tensor products with restriction functors [Kashiwara and Schapira 1990, Proposition 2.3.5]. On the other hand, any finite dimensional algebraic representation $V$ gives rise to a $D(G)$-module $M$, where $M=V^{\prime}$. If $V$ is a $U\left(\mathfrak{g}_{K}\right)_{\theta}$-module, then $M$ is a $D(G)_{\theta}$-module. In particular, the results of Section 8 allow us to associate to $M$ the $\mathscr{D}_{r, \chi}$-module $\mathscr{L}_{r, \chi}(M)$. Recall that this module is given by

$$
\mathscr{L}_{r, \chi}(M)=\mathscr{D}_{r, \chi} \otimes_{\underline{D}_{r, \theta}} \underline{M}_{r}
$$

Now the canonical morphism $\mathcal{D}_{\mathscr{B}, \chi}^{\text {an }} \rightarrow \mathscr{D}_{r, \chi}$ induces a morphism

$$
C^{B B}: \mathcal{D}_{\mathscr{B}, \chi}^{\mathrm{an}} \otimes_{U\left(\mathfrak{g}_{K}\right)_{\theta}} M \rightarrow \mathscr{D}_{r, \chi} \otimes_{\underline{D}_{r, \theta}} \underline{M}_{r}
$$

Recall that $r=r_{m}$ for some $m$.

Theorem 10.1.1. There is $r(M) \in\left[r_{0}, 1\right)$ such that for $r \geq r(M)$ (i.e., $m \gg 0$ sufficiently large) the canonical morphism

$$
C^{B B}: \Delta(M)_{\mathscr{B}}^{\text {an }} \stackrel{\sim}{\longrightarrow} \mathscr{L}_{r, \chi}(M)
$$

is an isomorphism of $\mathcal{D}_{\mathscr{B}, \chi}^{\mathrm{an}}$-modules.

Proof. Let $F$ be a facet in $\mathscr{B}$ such that $F \subseteq \overline{\mathscr{C}}$. By Proposition 4.2.10 of [Emerton 2011] the $D\left(U_{F}^{(e)}\right)$-module $M$ decomposes into a finite direct sum of irreducible $D\left(U_{F}^{(e)}\right)$-modules $M_{i}$. Since all $M_{i}$ are coadmissible $D\left(U_{F}^{(e)}\right)$-modules there exists $r(F) \in\left[r_{0}, 1\right)$ such that

$$
M_{i, r}:=D_{r}\left(U_{F}^{(e)}\right) \otimes_{D\left(U_{F}^{(e)}\right)} M_{i} \neq 0
$$

for all $r \geq r(F)$ and all $i$. By Theorem A in [Schneider and Teitelbaum 2002, Section 3] the $D\left(U_{F}^{(e)}\right)$-equivariant map $M_{i} \rightarrow M_{i, r}, m \mapsto 1 \otimes m$ has dense image and is therefore surjective. Since $M_{i}$ is irreducible the map is therefore bijective whenever $r \geq r(F)$. It follows $M \stackrel{\sim}{\longrightarrow} M_{r}\left(U_{F}^{(e)}\right)$ for $r \geq r(F)$. Given $g \in G$ we can use the $G$-equivariance of the sheaf $\underline{M}_{r}$ to express the canonical map 
$M \rightarrow M_{r}\left(U_{g^{-1} F}^{(e)}\right)$ as the composite

$$
M \stackrel{g .}{\longrightarrow} M \stackrel{\sim}{\longrightarrow} M_{r}\left(U_{F}^{(e)}\right) \stackrel{g^{*}}{\longrightarrow} M_{r}\left(U_{g^{-1} F}^{(e)}\right) .
$$

It is therefore bijective. Put $r(M):=\max _{F \subseteq \bar{C}} r(F)$. Then $M \stackrel{\sim}{\longrightarrow} M_{r}\left(U_{F}^{(e)}\right)$ for all $F \subset \mathscr{B}$ and all $r \geq r(M)$. Identifying $M$ with its constant sheaf on $\mathscr{B}$ the natural morphism $M \stackrel{\sim}{\longrightarrow} \underline{M}_{r}$ is therefore an isomorphism for all $r \geq r(M)$. On the other hand, arguing stalkwise gives, by Lemma 8.2.4, a canonical isomorphism

$$
\mathcal{D}_{\mathscr{B}, \chi}^{\text {an }} \otimes_{U\left(\mathfrak{g}_{K}\right)_{\theta}} \underline{M}_{r} \stackrel{\sim}{\longrightarrow} \mathscr{D}_{r, \chi} \otimes_{\underline{D}_{r, \theta}} \underline{M}_{r} .
$$

\section{A class of examples}

Throughout this section we suppose that the conditions (8.0.1) are fulfilled.

11.1.1. Let $\mathcal{O}$ be the classical BGG-category for the reductive Lie algebra $\mathfrak{g}_{K}$ relative to the choice of Borel subalgebra $\mathfrak{b}_{K}$ [Bernstein et al. 1976]. Since this category was originally defined for complex semisimple Lie algebras only we briefly repeat what we mean by it here. The category $\mathcal{O}$ equals the full subcategory of all (left) $U\left(\mathfrak{g}_{K}\right)$-modules consisting of modules $M$ such that

(i) $M$ is finitely generated as $U\left(\mathfrak{g}_{K}\right)$-module;

(ii) the action of $\mathfrak{t}_{K}$ on $M$ is semisimple and locally finite;

(iii) the action of $\mathfrak{n}_{K}$ on $M$ is locally finite.

Recall here that $\mathfrak{t}_{K}$ acts locally finitely on some module $M$ if $U\left(\mathfrak{t}_{K}\right) . m$ is finite dimensional for all $m \in M$ (similar for $\mathfrak{n}_{K}$ ).

Let $\mathcal{O}_{\text {alg }}$ be the full abelian subcategory of $\mathcal{O}$ consisting of those $U\left(\mathfrak{g}_{K}\right)$-modules whose $\mathfrak{t}_{K}$-weights are integral, i.e., are contained in the lattice $X^{*}(\boldsymbol{T}) \subset \mathfrak{t}_{K}^{*}$.

11.1.2. In [Orlik and Strauch 2010a] the authors study an exact functor

$$
M \mapsto \mathcal{F}_{B}^{G}(M)
$$

from $\mathcal{O}_{\text {alg }}$ to admissible locally analytic $G$-representations. It maps irreducible modules to (topologically) irreducible representations. The image of $\mathcal{F}_{B}^{G}$ comprises a wide class of interesting representations containing all principal series representations and many representations arising from homogeneous vector bundles on $p$-adic symmetric spaces. In this final section we wish to study the localizations of representations in this class. We restrict our attention to modules $M \in \mathcal{O}_{\text {alg, } \theta}$ having fixed central character $\theta$. Let $\chi \in \mathfrak{t}_{K}^{*}$ be such that $\sigma(\chi)=\theta$.

11.1.3. To start with let $U\left(\mathfrak{g}_{K}, B\right)$ be the smallest subring of $D(G)$ containing $U\left(\mathfrak{g}_{K}\right)$ and $D(B)$. The $\mathfrak{b}$-action on any $M \in \mathcal{O}_{\text {alg }}$ integrates to an algebraic, and 
hence, locally analytic $B$-action on $M$ and one has a canonical $D(G)$-module isomorphism

$$
\mathcal{F}_{B}^{G}(M)_{b}^{\prime} \stackrel{\sim}{\longrightarrow} D(G) \otimes_{U\left(\mathfrak{g}_{K}, B\right)} M=: N
$$

[Orlik and Strauch 2010a, Proposition 3.6]. Of course, $N$ is a $D(G)_{\theta}$-module. We may therefore consider its localization $\mathscr{L}_{r, \chi}(N)$ on $\mathscr{B}$. We recall that the stalk $\mathscr{L}_{r, \chi}(N)_{z}$ at a point $z$ is a quotient of $\kappa(z) \hat{\otimes}\left(\underline{N}_{r}\right)_{z}$ (see Proposition 8.2.2) and therefore has its quotient topology. We finally say a morphism of sheaves to $\mathscr{L}_{r, \chi}(N)$ has dense image if this holds stalkwise at all points.

On the other hand, we may form

$$
G M:=K[G] \otimes_{K[B]} M .
$$

It may be viewed as a $U\left(\mathfrak{g}_{K}\right)$-module via $x .(g \otimes m):=g \otimes \operatorname{Ad}\left(g^{-1}\right)(x) . m$ for $g \in G, m \in M, x \in \mathfrak{g}_{K}$. Since $K[G]$ is a free right $K[B]$-module, $G M$ equals the direct sum of $U\left(\mathfrak{g}_{K}\right)$-submodules $g M:=g \otimes M$ indexed by a system of coset representatives $g$ for $G / B$. Since the group $G$ is connected, the adjoint action of $G=\boldsymbol{G}(L)$ fixes the center $Z\left(\mathfrak{g}_{K}\right) \subset U\left(\mathfrak{g}_{K}\right)$ [Demazure and Gabriel 1970, II, Section 6.1.5] and therefore $G M$ still has central character $\theta$. Let us consider its Beilinson-Bernstein module $\Delta(G M)$ over $X$. The linear map $g M \stackrel{\sim}{\longrightarrow} M$ given by $g \otimes m \mapsto m$ is an isomorphism and equivariant with respect to the automorphism $\operatorname{Ad}\left(g^{-1}\right)$ of $U\left(\mathfrak{g}_{K}\right)$. It follows that, given an open subset $V \subseteq X$, we have a linear isomorphism $\Delta(g M)(V) \stackrel{\sim}{\longrightarrow} \Delta(M)\left(g^{-1} V\right)$ given by $\delta \otimes(g \otimes m) \mapsto g^{*}(\delta) \otimes m$ for a local section $\delta$ of $\mathcal{D}_{\chi}$ and $m \in M$. Here $g^{*}$ refers to the $G$-equivariant structure on $\mathcal{D}_{\chi}$ 8.3.2. The same argument works for the analytifications $\Delta^{\text {an }}(g M)$ and $\Delta^{\text {an }}(M)$. In particular, the stalks $\Delta^{\mathrm{an}}(g M)_{z} \simeq \Delta^{\mathrm{an}}(M)_{g^{-1} z}$ are isomorphic vector spaces for any $z \in \mathscr{B}$ and any $g \in G$.

Lemma 11.1.4. $\left.\quad \Delta^{\mathrm{an}}(M)\right|_{\mathscr{B}}=\left.0 \Longleftrightarrow \Delta^{\mathrm{an}}(G M)\right|_{\mathscr{B}}=0$.

Proof. Suppose $\left.\Delta^{\text {an }}(M)\right|_{\mathscr{B}}=0$. Let $g \in G$. For any $z \in \mathscr{B}$ we compute $\Delta^{\text {an }}(g M)_{z} \simeq$ $\Delta^{\text {an }}(M)_{g^{-1} z}=0$, whence $\left.\Delta^{\text {an }}(g M)\right|_{\mathscr{B}}=0$. This yields $\left.\Delta^{\text {an }}(G M)\right|_{\mathscr{B}}=0$, since $\Delta^{\mathrm{an}}(\cdot)_{\mathscr{B}}$ commutes with arbitrary direct sums. The converse is clear.

Lemma 11.1.5. There is a canonical morphism of $\mathscr{D}_{r, \chi}$-modules

$$
\mathscr{D}_{r, \chi} \otimes_{\mathcal{D}_{\mathscr{B}, \chi}^{\mathrm{an}}} \Delta(G M)_{\mathscr{B}}^{\mathrm{an}} \rightarrow \mathscr{L}_{r, \chi}\left(\mathcal{F}_{B}^{G}(M)^{\prime}\right)
$$

functorial in $M$ and with dense image.

Proof. The morphism is induced from the functorial map

$$
P: G M \rightarrow D(G) \otimes_{U\left(\mathfrak{g}_{K}, B\right)} M=N
$$

via the inclusions $K[B] \subset D(B)$ and $K[G] \subset D(G)$. Let us show that the morphism has dense image. We claim first that the map $P$ has dense image with respect to 
the canonical topology on the coadmissible module $N$. Let $G_{0}$ be the (hyper-) special maximal compact open subgroup of $G$ equal to the stabilizer of the origin $x_{0} \in A$. Let $B_{0}:=B \cap G_{0}$. The Iwasawa decomposition $G=G_{0} \cdot B$ implies $K[G]=K\left[G_{0}\right] \otimes_{K\left[B_{0}\right]} K[B]$ and similarly for distributions $D(\cdot)$. Let $G_{0} M:=$ $K\left[G_{0}\right] \otimes_{K\left[B_{0}\right]} M$ and $N_{0}:=D\left(G_{0}\right) \otimes_{U\left(\mathfrak{g}_{K}, B_{0}\right)} M$. Then $G_{0} M \simeq G M$ as $K\left[G_{0}\right]-$ modules and $N \simeq N_{0}$ as $D\left(G_{0}\right)$-modules via the obvious maps. Write $D\left(G_{0}\right)=$ $\lim _{r} D_{r}\left(G_{0}\right)$ with some Banach algebra completions $D_{r}\left(G_{0}\right)$. The map $P$ induces maps $P_{r}: G_{0} M \rightarrow D_{r}\left(G_{0}\right) \otimes_{U\left(\mathfrak{g}_{K}, B_{0}\right)} M$. Since $K\left[G_{0}\right] \subset D_{r}\left(G_{0}\right)$ is dense, the definition of the Banach topology on the target implies that $P_{r}$ has dense image. Passing to the limit over $r$ shows that $P$ has dense image. Let $z \in \mathscr{B}$. Then the map $P$ composed with the map $N \rightarrow \underline{N}_{r, z}$ has dense image [Schneider and Teitelbaum 2003, Section 3, Theorem A]. Now we are done: the map

$$
\mathscr{D}_{r, \chi, z} \otimes_{\mathcal{D}_{\mathscr{B}, \chi, z}^{\mathrm{an}}} \Delta(G M)_{\mathscr{B}, z}^{\mathrm{an}} \rightarrow \mathscr{L}_{r, \chi}(N)_{z},
$$

pulled back to $\Delta(G M)_{\mathscr{B}, z}^{\mathrm{an}}$, may be written as

$$
\begin{aligned}
&\left(\left(\kappa(z) \hat{\otimes}_{L} G M\right) / \mathfrak{n}_{\pi(z)}\left(\kappa(z) \hat{\otimes}_{L} G M\right)\right)_{\lambda \text {-coinv }} \rightarrow \\
& \quad\left(\left(\kappa(z) \hat{\otimes}_{L} \underline{N}_{r, z}\right) / \mathfrak{n}_{\pi(z)}\left(\kappa(z) \hat{\otimes}_{L} \underline{N}_{r, z}\right)\right)_{\lambda \text {-coinv }}
\end{aligned}
$$

by Theorem 5.2.2 and Proposition 8.2.2. Consequently, it has dense image.

11.1.6. We now look closer at the case $\theta=\theta_{0}$ and $\chi=\rho$. Let $V:=\operatorname{ind}_{B}^{G}(1)$ be the smooth induction of the trivial character of $B$. Its smooth dual $\check{V}$ equals the smooth induction $\operatorname{ind}_{B}^{G}\left(\delta_{B}^{-1}\right)$, where $\delta_{B}: B \rightarrow \mathbb{Q}^{\times} \subseteq K^{\times}$is the modulus character of the locally compact group $B$. We choose $e$ large enough so that the Schneider-Stuhler sheaf $\underset{\approx}{\check{V}}$ of $\check{V}$ is nonzero [Schneider and Stuhler 1997, Theorem IV.4.1].

The finitely many irreducible modules in $\mathcal{O}_{\mathrm{alg}, \theta_{0}}$ are given by the irreducible quotients $L_{w}$ of the Verma modules $M_{w}$ of highest weight $-w(\rho)-\rho$ for $w \in W$. The cardinality of the latter set of weights is $|W|$. As usual, $w_{0}$ denotes the longest element in $W$. Let $w \in W$. Let $\mathcal{M}_{w}$ and $\mathcal{L}_{w}$ be the Beilinson-Bernstein localizations over $X$ of $M_{w}$ and $L_{w}$ respectively. Let $\iota_{w}: X_{w} \hookrightarrow X$ be the inclusion of the Bruhat cell $\boldsymbol{B} w \boldsymbol{B} / \boldsymbol{B}$ into $X$ and let $\mathcal{O}_{X_{w}}$ be its structure sheaf with its natural (left) $D_{X_{w}}{ }^{-}$ module structure. Let $\mathcal{N}_{w}=\iota_{w *} \mathcal{O}_{w}$ be its $D$-module push-forward to $X$. Since $\mathcal{O}_{X_{w}}$ is a holonomic module and $\iota_{w}$ is an affine morphism, $\mathcal{N}_{w}$ may be viewed as an $D_{X}$-module (rather than just a complex of such) [Hotta et al. 2008, 3.4].

Proposition 11.1.7. Let $w \in W$ and $\mathcal{L}_{w}^{\mathrm{an}}$ be the analytification of $\mathcal{L}_{w}$. Then $\left.\mathcal{L}_{w_{0}}^{\mathrm{an}}\right|_{\mathscr{B}}=$ $\mathcal{O}_{\mathscr{B}}$ and $\left.\mathcal{L}_{w}^{\text {an }}\right|_{\mathscr{B}}=0$ for $w \neq w_{0}$.

Proof. By [loc. cit., Lemma 12.3.1] the sheaf $\mathcal{N}_{w}$ has support contained in $X_{w}$. By [loc. cit., Proposition 12.3.2(i)] the module $\mathcal{L}_{w}$ injects into $\mathcal{N}_{w}$. Now let $w \neq w_{0}$. Let $\eta \in X$ be the generic point of $X$ and $X_{\eta}^{\text {an }}$ the fiber of $\pi: X^{\text {an }} \rightarrow X$ over $\eta$. Since 
$\eta \notin X_{w}$ one has $\left(\mathcal{N}_{w}\right)_{\eta}=0$ and therefore $\left.\mathcal{N}_{w}^{\mathrm{an}}\right|_{X_{\eta}^{\mathrm{an}}}=0$. Lemma 6.2.2 states that $\mathscr{B} \subset X_{\eta}^{\text {an }}$ whence $\left.\mathcal{L}_{w}^{\text {an }}\right|_{\mathscr{B}}=0$. The converse is clear: the module $L_{w_{0}}$ equals the trivial one-dimensional $U(\mathfrak{g})$-module having localization $\mathcal{L}_{w_{0}}=\mathcal{O}_{X}$ (e.g., by the Borel-Weil theorem). Hence, $\left.\mathcal{L}_{w_{0}}^{\text {an }}\right|_{\mathscr{B}}=\mathcal{O}_{\mathscr{B}}$.

Corollary 11.1.8. Let $w \in W$. Then $\mathscr{L}_{r, \rho}\left(\mathcal{F}_{B}^{G}\left(L_{w}\right)^{\prime}\right) \neq 0$ if and only if $w=w_{0}$.

Proof. Let $w \neq w_{0}$. The preceding proposition together with the first lemma above yields $\left.\Delta^{\text {an }}\left(\mathrm{GL}_{w}\right)\right|_{\mathscr{B}}=0$. The second lemma then yields $\mathscr{L}_{r, \rho}\left(\mathcal{F}_{B}^{G}\left(L_{w}\right)^{\prime}\right)=0$. Conversely, let $w=w_{0}$. We have $\mathcal{F}_{B}^{G}\left(L_{w_{0}}\right)=\operatorname{ind}_{B}^{G}(1)=V$, the smooth induction of the trivial $B$-representation [Orlik and Strauch 2010a]. By the choice of $e$ we have $\underset{\approx}{\check{V}} \neq 0$. Let $z \in \mathscr{B}$ be a point such that $\check{V}_{U_{z}^{(e)}} \neq 0$. With $N:=V^{\prime}$ and $\left(U_{z}^{(e)}\right)_{(r)}:=U_{z}^{(e)} \cap U_{r}\left(U_{z}^{(e)}\right)$, Proposition 9.1.3 yields a surjection

$$
\left(\underline{N}_{r}\right)_{z}=D_{r}\left(U_{z}^{(e)}\right) \otimes_{D\left(U_{z}^{(e)}\right)} N=\check{V}_{\left(U_{z}^{(e)}\right)_{(r)}} \rightarrow \check{V}_{U_{z}^{(e)}}
$$

between the two spaces of coinvariants which implies $\left(\underline{N}_{r}\right)_{z} \neq 0$. It follows that $\mathscr{L}_{r, \rho}(N)_{z}=\kappa(z) \otimes_{L}\left(\underline{N}_{r}\right)_{z} \neq 0$ (Proposition 8.2.2), which means $\left.\mathscr{L}_{r, \rho}(N)\right|_{\mathscr{B}} \neq 0$.

Recall that any $U\left(\mathfrak{g}_{K}\right)$-module $M \in \mathcal{O}$ is of finite length.

Proposition 11.1.9. Let $M \in \mathcal{O}_{\mathrm{alg}, \theta_{0}}$. Let $n \geq 0$ be the Jordan-Hölder multiplicity of the trivial representation in the module $M$ and let $V=\operatorname{ind}_{B}^{G}(1)$. There is a (noncanonical) isomorphism of $\mathcal{O}_{\mathscr{B}}$-modules

$$
\mathscr{L}_{\rho, r}\left(\mathcal{F}_{B}^{G}(M)^{\prime}\right) \stackrel{\sim}{\longrightarrow} \mathscr{L}_{\rho, r}\left(V^{\oplus \oplus n}\right)
$$

with both sides equal to zero in case $n=0$.

Proof. Let $\underset{\approx}{\check{V}}, r$ be the constructible sheaf of $K$-vector spaces on $\mathscr{B}$ which is constructed in the same way as $\underset{\sim}{\mathscr{V}}$ but using the groups $\left(U_{F}^{(e)}\right)_{(r)}$ instead of $U_{F}^{(e)}$ for all facets $F$. The very same arguments as in the case $r=r_{0}$ (Theorem 9.2.5) show that the $\mathcal{O}_{\mathscr{B}}$-module $\mathscr{L}_{\rho, r}\left(V^{\prime}\right)$ is isomorphic to the module $\mathcal{O}_{\mathscr{B}} \otimes_{L} \check{\sim}_{, r}$. In particular, it is a free $\mathcal{O}_{\mathscr{B}}$-module.

We now prove the claim of the proposition by induction on $n$. Let $n=0$. By exactness of the functors $\mathcal{F}_{B}^{G}(\cdot)^{\prime}$ and $\mathscr{L}_{\rho, r}$ a Jordan-Hölder filtration of $M$ induce a filtration of $\mathscr{L}_{\rho, r}\left(\mathcal{F}_{B}^{G}(M)^{\prime}\right)$ whose graded pieces vanish by the preceding corollary. Thus $\mathscr{L}_{\rho, r}\left(\mathcal{F}_{B}^{G}(M)^{\prime}\right)=0$. Let $n=1$. Using a Jordan-Hölder filtration of $M$ and the case $n=0$ we may assume that the trivial representation sits in the top graded piece of $M$. Applying the case $n=0$ a second time gives the claim. Assume now $n \geq 2$. Using again a Jordan-Hölder filtration of $M$ we have an exact sequence

$$
0 \rightarrow M_{1} \rightarrow M \rightarrow M_{2} \rightarrow 0
$$


in $\mathcal{O}_{\text {alg, } \theta_{0}}$, where $M_{i}$ has multiplicity $n_{i} \geq 1$. Applying the induction hypothesis to $M_{1}$ and $M_{2}$ yields an exact sequence of $\mathcal{O}_{\mathscr{B}}$-modules

$$
0 \rightarrow \mathscr{L}_{\rho, r}\left(V^{\prime \oplus n_{1}}\right) \rightarrow \mathscr{L}_{\rho, r}\left(\mathcal{F}_{B}^{G}(M)^{\prime}\right) \rightarrow \mathscr{L}_{\rho, r}\left(V^{\prime \oplus n_{2}}\right) \rightarrow 0 .
$$

By our first remark this sequence is (noncanonically) split. Since $\mathscr{L}_{\rho, r}$ commutes with direct sums, this completes the induction.

When $r=r_{0}$ the statement of the preceding Proposition 11.1.9 can be made more concrete, because in this case the sheaf $\mathscr{L}_{\rho, r_{0}}\left(V^{\prime \oplus n}\right)$ equals the sum over $n$ copies of $\mathcal{O}_{\mathscr{B}} \otimes_{L} \underset{\approx}{\check{V}}$ with $\check{V}=\operatorname{ind}_{B}^{G}\left(\delta_{B}^{-1}\right)$; see Theorem 9.2.5.

\section{Appendix: Analyticity of group actions near points on the building}

In this appendix we give a proof of Lemma 6.2.6 about the analyticity of group actions near points on the building. Before doing so we would like to remark that we have not used anything special about these points, except that they correspond to supremum norms on affinoid subdomains. It is certainly possible to prove more general statements in similar settings.

Proof of Lemma 6.2.6. Step 1. Recall that $G$ acts transitively on the set of apartments in $\mathscr{B}$, and that we denote by $A=\left(X_{*}(\boldsymbol{T}) / X_{*}(\boldsymbol{C})\right) \otimes_{\mathbb{Z}} \mathbb{R}$ the apartment which corresponds to the torus $\boldsymbol{T}$; see 4.1.2. The affine Weyl group determined by $\boldsymbol{T}$ acts on $A$, and this action has a relatively compact fundamental domain, which we denote by $D$. Because of the identity $g U_{z}^{(e)} g^{-1}=U_{g z}^{(e)}$ in (4.1.6), it suffices to prove the assertion of Lemma 6.2.6 for those $z$ which lie in the closure $\bar{D}$ of $D$. For any fixed $e$, the set of groups $\left\{U_{z}^{(e)} \mid z \in \bar{D}\right\}$ is finite, as $\bar{D}$ is compact. Recall that for fixed $z$ the groups $U_{z}^{(e)}$ form a fundamental system of neighborhoods of 1 in $G$; see Proposition 4.1.7. Hence, given any affinoid subgroup $\mathbb{U} \subset G^{\text {an }}$, there is $e_{\mathrm{st}} \geq e_{\mathrm{uni}}$ such that $\mathbb{U}_{z}^{(e)} \subset \mathbb{U}(L)$ for all $z \in \bar{D}$ and all $e \geq e_{\mathrm{st}}$. In Step 3 below we exhibit a certain condition for an affinoid subgroup $\mathbb{U} \subset \boldsymbol{G}^{\text {an }}$. This condition is fulfilled by any sufficiently small affinoid subgroup $\mathbb{U}$. We will then show that there is a fundamental system $\left\{V_{n}\right\}_{n \geq 0}$ as in Lemma 6.2.4 such that $\mathbb{U}$ acts analytically on every $V_{n}$, in the sense of Section 6.2.5.

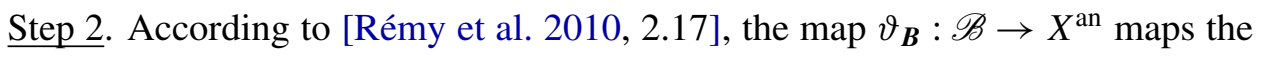
apartment $A$ into the analytification of the open subscheme $U_{1}=N^{-} \boldsymbol{B} / \boldsymbol{B} \subset X$, which is isomorphic to $\boldsymbol{N}^{-}$(notation as in 5.1.3, 5.1.5). Put $\Psi=-\Phi^{+}(\boldsymbol{G}, \boldsymbol{T})$. The choice of a Chevalley basis for $\mathfrak{g}$ gives coordinates $\left(X_{\alpha}\right)_{\alpha \in \Psi}$ on $N^{-}$, hence on $U_{1}$. The points of the apartment $A$, considered as a subset of $U_{1}^{\text {an }}$, can then be described as norms on the algebra $L\left[\left(X_{\alpha}\right)_{\alpha \in \Psi}\right]=\mathcal{O}_{X}\left(U_{1}\right)$ as follows. To $z \in A$ there corresponds the norm 


$$
L\left[\left(X_{\alpha}\right)_{\alpha \in \Psi}\right] \ni \sum_{\nu \in \mathbb{N}^{\Psi}} a_{\nu} X^{\nu} \mapsto \sup _{\nu}\left|a_{\nu}\right| \prod_{\alpha \in \Psi} e^{\nu(\alpha)\langle z, \alpha\rangle} ;
$$

see [Rémy et al. 2010, 2.17]. Here $\langle z, \alpha\rangle$ is the canonical pairing between cocharacters and characters. The norm just described is the supremum norm on the polydisc $\mathbb{D}(r)$ with polyradius $r=\left(e^{\langle z, \alpha\rangle}\right)_{\alpha \in \Psi} . \mathbb{D}(r)$ is an affinoid domain in $U_{1}^{\text {an }}$, and it is strictly affinoid if (and only if) all $e^{\langle z, \alpha\rangle}$ are in the extended value group $\sqrt{\left|L^{*}\right|}$. As $\bar{D}$ is a compact subset of $A$, there are numbers $R_{0}>1>r_{0}$ in $\left|L^{*}\right|$ such that

$$
r_{0}<\inf _{\substack{z \in \bar{D} \\ \alpha \in \Psi}} e^{\langle z, \alpha\rangle} \quad \text { and } \quad \sup _{\substack{z \in \bar{D} \\ \alpha \in \Psi}} e^{\langle z, \alpha\rangle}<R_{0} .
$$

In particular, $\bar{D}$ lies in the interior of the (strictly) affinoid polydisc $\mathbb{D}\left(R_{0}\right)$ with polyradius $\left(R_{0}, \ldots, R_{0}\right)$. By [Berkovich $\left.1990,3.4 .6\right], U_{1}^{\text {an }}$ is an open subset of $X^{\text {an }}$. The polydisc $\mathbb{D}\left(R_{0}\right)$ is thus a neighborhood of $\bar{D}$. Because of this we will henceforth work on $\mathbb{D}\left(R_{0}\right)$.

$\underline{\text { Step 3 }}$. Let $\mathbb{C}_{p}$ be the completion of an algebraic closure of $L$. Let $\|\cdot\|$ be the maximum norm on $U_{1}^{\text {an }}\left(\mathbb{C}_{p}\right)=\mathbb{C}_{p}^{\Psi}$, i.e., $\left\|\left(x_{\alpha}\right)_{\alpha \in \Psi}\right\|=\max _{\alpha}\left|x_{\alpha}\right|$. Fix $r_{1} \in\left(0, r_{0}\right)$. We claim that there is a connected strictly affinoid subgroup $\mathbb{U} \subset G^{\text {an }}$ which leaves $\mathbb{D}\left(R_{0}\right)$ stable, and such that

for all $g \in \mathbb{U}\left(\mathbb{C}_{p}\right)$ and all $x \in \mathbb{D}\left(R_{0}\right)\left(\mathbb{C}_{p}\right)$ one has $\|g(x)-x\| \leq r_{1}$.

To see this, let $\Lambda^{-} \subset \operatorname{Lie}\left(\boldsymbol{N}^{-}\right)$and $\Lambda^{+} \subset \mathfrak{b}$ be $o_{L}$-lattices, and put $\Lambda_{m}^{-}=p^{m} \Lambda^{-}$ and $\Lambda_{m}^{+}=p^{m} \Lambda^{+}$. For $m$ large enough $\Lambda_{m}^{-}, \Lambda_{m}^{+}$, and $\Lambda_{m}:=\Lambda_{m}^{-} \oplus \Lambda_{m}^{+}$will be $o_{L}$-Lie subalgebras. After possibly increasing $m$, these lattices can be exponentiated to give good analytic subgroups ${ }^{8} \mathbb{U}_{m}^{-}=\exp _{G}\left(\Lambda_{m}^{-}\right)^{\text {an }} \subset N^{- \text {an }}, \mathbb{U}_{m}^{+}=\exp _{G}\left(\Lambda_{m}^{+}\right)^{\text {an }} \subset \boldsymbol{B}^{\text {an }}$, and $\mathbb{U}_{m}=\exp _{G}\left(\Lambda_{m}\right)^{\text {an }} \subset \boldsymbol{G}^{\text {an }}$. Increasing $m$ further (if necessary) ensures that $\mathbb{U}_{m}$ has an Iwahori decomposition, i.e., $\mathbb{U}_{m}=\mathbb{U}_{m}^{-} \times \mathbb{U}_{m}^{+}$(this follows from the existence of "coordinates of the second kind"). Next consider $\mathbb{D}\left(R_{0}\right)$ as an affinoid subdomain in the group $N^{- \text {,an }}$, the analytification of $N^{-}$. Then, for any given positive integer $m_{1}$ there will be $m_{2} \gg m_{1}$ such that $x^{-1} \mathbb{U}_{m_{2}} x \subset \mathbb{U}_{m_{1}}$ for all $x \in \mathbb{D}\left(R_{0}\right)$, because $\mathbb{D}\left(R_{0}\right) \subset \boldsymbol{N}^{- \text {,an }}$ is bounded. Furthermore, because $\mathbb{D}\left(R_{0}\right)$ is defined in terms of the Chevalley basis, we can find $m_{1}$ such that $\mathbb{D}\left(R_{0}\right)$ (as a subset in $N^{- \text {an }}$ ) is stable by right multiplication by $\mathbb{U}_{m_{1}}^{-}$. Enlarging $m_{1}$ if necessary we even have $\|x h-x\| \leq r_{1}$ for all $x \in \mathbb{D}\left(R_{0}\right)$ and $h \in \mathbb{U}_{m_{1}}^{-}$(each coordinate $X_{\alpha}$ of $x h$ will be very close to that of $x$ if $h$ is close to the identity). Now fix $g \in \mathbb{U}_{m_{2}}$ and $x \in \mathbb{D}\left(R_{0}\right)$, considered as an element of $\boldsymbol{N}^{- \text {an }}$. Write $x^{-1} g x=u^{-} u^{+}$with $u^{-} \in \mathbb{U}_{m_{1}}^{-}$and $u^{+} \in \mathbb{U}_{m_{1}}^{+} \subset \boldsymbol{B}^{\text {an }}$. Then, as elements of $U_{1}^{\text {an }} \subset X^{\text {an }}$ we have $g(x)=g x \boldsymbol{B}^{\text {an }}=x u^{-} u^{+} \boldsymbol{B}^{\text {an }}=x u^{-} \boldsymbol{B}^{\text {an }}$. This shows that $\mathbb{D}\left(R_{0}\right)$ is stable under the left action of $\mathbb{U}_{m_{2}}$ and the inequality in

\footnotetext{
${ }^{8}$ In the sense of [Emerton 2011].
} 
(11.1.10) is satisfied. With this choice of $m_{2}$ we let $\mathbb{U}=\mathbb{U}_{m_{2}}$. We put

$$
\delta=\frac{r_{1}}{\inf _{z \in \bar{D}, \alpha \in \Psi} e^{\langle z, \alpha\rangle}},
$$

which is less than 1 .

Step 4. From now on we fix a point $z \in \bar{D}$, which we think of as a supremum norm on the polydisc $\mathbb{D}(r)$ with polyradius $r=\left(e^{\langle z, \alpha\rangle}\right)_{\alpha \in \Psi}$. We remark that $\left|X_{\alpha}(z)\right|=e^{\langle z, \alpha\rangle}$. It follows from the very definition of the topology on the affinoid space $\mathbb{D}\left(R_{0}\right)$ that a fundamental system of neighborhoods of $z$ is given by finite intersections of sets of the form

$$
V_{f, c, C}=\left\{x \in \mathbb{D}\left(R_{0}\right)|c \leq| f(x) \mid \leq C\right\},
$$

where $f \in \mathcal{O}\left(\mathbb{D}\left(R_{0}\right)\right)=L\left\langle R_{0}^{-1} X\right\rangle$ and $c<|f(z)|<C$ [Berkovich 1990, 2.2.3(iii)]. A particular example of such a neighborhood is the annulus

$$
\mathbb{A}_{s, t}=\left\{x \in \mathbb{D}\left(R_{0}\right)\left|\forall \alpha \in \Psi: s_{\alpha} \leq\right| X_{\alpha}(x) \mid \leq t_{\alpha}\right\}=\bigcap_{\alpha} V_{X_{\alpha}, s_{\alpha}, t_{\alpha}},
$$

where $s=\left(s_{\alpha}\right)_{\alpha \in \Psi}$ and $t=\left(t_{\alpha}\right)_{\alpha \in \Psi}$ are such that $s_{\alpha}<e^{\langle z, \alpha\rangle}<t_{\alpha}$ for all $\alpha \in \Psi$.

Let $\underline{r}_{0}=\left(r_{0}, \ldots, r_{0}\right)$ be the tuple indexed by $\Psi$ which has all components equal to $r_{0}$. Given a neighborhood $V_{f, c, C}$, we are now going to find real numbers $c^{\prime}<C^{\prime}$ in $\sqrt{\left|L^{*}\right|}$, and a tuple $r^{\prime}=\left(r_{\alpha}^{\prime}\right)_{\alpha \in \Psi} \in \sqrt{\left|L^{*}\right|} \Psi$ such that

$$
V^{\prime}=V_{f, c^{\prime}, C^{\prime}} \cap \mathbb{A}_{\underline{r}_{0}, r^{\prime}}
$$

(i) is a neighborhood of $z$,

(ii) is contained in $V_{f, c, C}$, and

(iii) is stable under the action of $\mathbb{U}$.

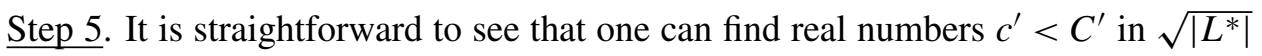
with the properties

$$
c<c^{\prime}<|f(z)|<C^{\prime}<C \text { and } C^{\prime} \delta<c^{\prime},
$$

where $\delta$ is as in Step 3. Furthermore, as $f$ has supremum norm less than $C^{\prime}$ on the disk $\mathbb{D}(r)$, we can find $r^{\prime}=\left(r_{\alpha}^{\prime}\right)_{\alpha \in \Psi} \in{\sqrt{\left|L^{*}\right|}}^{\Psi}$ such that

- for all $\alpha \in \Psi: r_{\alpha}^{\prime}>e^{\langle z, \alpha\rangle}$,

- $f$ has supremum norm less or equal to $C^{\prime}$ on the $\operatorname{disc} \mathbb{D}\left(r^{\prime}\right)$.

We remark that the affinoid group $\mathbb{U}$ acts on the strictly affinoid annulus $\mathbb{A}_{\underline{r}_{0}, r^{\prime}}$, because $r_{1}<r_{0}$. Moreover, the strictly affinoid domain $V^{\prime}=V_{f, c^{\prime}, C^{\prime}} \cap \mathbb{A}_{\underline{r}_{0}, r^{\prime}}$ is a neighborhood of $z$. Our aim is to show that $\mathbb{U}$ also acts on $V^{\prime}$. To see this, it is enough to work with $\mathbb{C}_{p}$-valued points. Write $f$ as a power series, $f(X)=\sum_{\nu \in \mathbb{N}^{\Psi}} a_{\nu} X^{v}$. 
Then we have $\left|a_{v}\right|\left(r^{\prime}\right)^{v} \leq C^{\prime}$. Consider $x \in V^{\prime}\left(\mathbb{C}_{p}\right)$ and $g \in \mathbb{U}\left(\mathbb{C}_{p}\right)$. Expand $f$ around $x$

$$
f\left(x^{\prime}\right)=f(x)+\sum_{\nu \neq 0} b_{v}\left(x^{\prime}-x\right)^{\nu} .
$$

Then we also have $\left|b_{\nu}\right|\left(r^{\prime}\right)^{\nu} \leq C^{\prime}$ for all $\nu$. Put $x^{\prime}=g(x)$ and get $f(g(x))=$ $f(x)+\sum_{\nu \neq 0} b_{\nu}(g(x)-x)^{v}$. Using the inequality $\|g(x)-x\| \leq r_{1}$ we find

$$
\left|b_{\nu}(g(x)-x)^{\nu}\right|=\left|b_{\nu}\right|\left(r^{\prime}\right)^{\nu} \frac{|g(x)-x|^{\nu}}{\left(r^{\prime}\right)^{\nu}} \leq C^{\prime} \cdot \frac{r_{1}^{|\nu|}}{\left(r^{\prime}\right)^{\nu}}<C^{\prime} \delta<c^{\prime} .
$$

We conclude that $|f(g(x))-f(x)|<c^{\prime}$ and thus

$$
|f(g(x))|=|f(x)+f(g(x))-f(x)|=|f(x)| .
$$

This shows that $\mathbb{U}$ acts on the (strictly) affinoid neighborhood $V^{\prime}$.

Step 6. In the general case, consider a neighborhood of $z$ of the form $V=V_{1} \cap \cdots \cap$ $V_{m}$ with $V_{i}=V_{f_{i}, c_{i}, C_{i}}$. Then we find for each $V_{i}$ a neighborhood $V_{i}^{\prime}$ stable under $\mathbb{U}$, as in Step 5. The intersection $V^{\prime}=V_{1}^{\prime} \cap \cdots \cap V_{m}^{\prime}$ will then be a neighborhood which is stable by the action of $\mathbb{U}$.

Step 7. Now let $W_{1} \supset W_{2} \supset \cdots$ be a sequence of neighborhoods of $z$ as in Lemma 6.2.4. Use Step 6 to find an strictly affinoid neighborhood $W_{1}^{\prime} \subset W_{1}$ of $z$ on which $\mathbb{U}$ acts. $W_{1}^{\prime}$ is not necessarily irreducible. But irreducible and connected components coincide here [Berkovich 1990, 3.1.8] (use that $X^{\text {an }}$ is a normal space, by [loc. cit., 3.4.3]), and $\mathbb{U}$, being connected, will stabilize the connected component of $W_{1}^{\prime}$ containing $z$. Call this connected component $V_{1}$. It is again a strictly affinoid neighborhood of $z$. Then choose $n$ such that $W_{n}$ is contained in the topological interior of $V_{1}$, and let $W_{n}^{\prime} \subset W_{n}$ be a neighborhood of $z$ on which $\mathbb{U}$ acts (by Step 6). Let $V_{2}$ be the connected component of $W_{n}^{\prime}$ containing $z$. Continuing this way we construct from $\left(W_{n}\right)_{n}$ a descending sequence of irreducible strictly affinoid neighborhoods $\left(V_{n}\right)_{n}$ with the same properties as that in Lemma 6.2.4, but with the additional property that $\mathbb{U}$ acts on each $V_{n}$. This finishes the proof of Lemma 6.2.6.

\section{Acknowledgments}

We thank Vladimir Berkovich for helpful correspondence on $p$-adic symmetric spaces and buildings. Furthermore, we thank an anonymous referee for pointing out inaccuracies in an earlier version and many comments which helped improve the paper in several places. Schmidt gratefully acknowledges travel support by the SFB 878 "Groups, Geometry \& Actions" at the University of Münster. Patel would like to thank Indiana University, Bloomington, for its support and hospitality. 


\section{References}

[Ardakov and Wadsley 2013] K. Ardakov and S. Wadsley, "On irreducible representations of compact p-adic analytic groups", Ann. of Math. (2) 178:2 (2013), 453-557. MR 3071505 Zbl 1273.22014

[Beilinson and Bernstein 1981] A. Beilinson and J. Bernstein, "Localisation de $g$-modules", C. R. Acad. Sci. Paris Sér. I Math. 292:1 (1981), 15-18. MR 82k:14015 Zbl 0476.14019

[Berkovich 1990] V. G. Berkovich, Spectral theory and analytic geometry over non-Archimedean fields, Mathematical Surveys and Monographs 33, Amer. Math. Soc., Providence, RI, 1990. MR 91k:32038 Zbl 0715.14013

[Berkovich 1993] V. G. Berkovich, "Étale cohomology for non-Archimedean analytic spaces", Inst. Hautes Études Sci. Publ. Math. 78 (1993), 5-161. MR 95c:14017 Zbl 0804.32019

[Bernstein et al. 1976] I. N. Bernstein, I. M. Gelfand, and S. I. Gelfand, "A certain category of $\mathfrak{g}$-modules”, Funkcional. Anal. i Priložen. 10:2 (1976), 1-8. In Russian; translated in Funct. Anal. Appl. 10:1 (1976), 87-92. MR 53 \#10880 Zbl 0353.18013

[Bezrukavnikov et al. 2008] R. Bezrukavnikov, I. Mirković, and D. Rumynin, "Localization of modules for a semisimple Lie algebra in prime characteristic", Ann. of Math. (2) 167:3 (2008), 945-991. MR 2009e:17031 Zbl 1220.17009

[Borel et al. 1987] A. Borel, P.-P. Grivel, B. Kaup, A. Haefliger, B. Malgrange, and F. Ehlers, Algebraic D-modules, Perspectives in Mathematics 2, Academic Press, Boston, 1987. MR 89g:32014 Zbl 0642.32001

[Bourbaki 1972] N. Bourbaki, Groupes et algèbres de Lie, Chapitres II/III, Actualités Scientifiques et Industrielles 1349, Hermann, Paris, 1972. MR 58 \#28083a Zbl 0244.22007

[Bourbaki 1987] N. Bourbaki, Topological vector spaces, Chapters 1-5, Springer, Berlin, 1987. MR 88g:46002 Zbl 0622.46001

[Bruhat and Tits 1972] F. Bruhat and J. Tits, "Groupes réductifs sur un corps local", Inst. Hautes Études Sci. Publ. Math. 41 (1972), 5-251. MR 48 \#6265 Zbl 0254.14017

[Bruhat and Tits 1984] F. Bruhat and J. Tits, "Groupes réductifs sur un corps local, II: Schémas en groupes: Existence d'une donnée radicielle valuée”, Inst. Hautes Études Sci. Publ. Math. 60 (1984), 197-376. MR 86c:20042 Zbl 0597.14041

[Brylinski and Kashiwara 1980] J.-L. Brylinski and M. Kashiwara, "Démonstration de la conjecture de Kazhdan-Lusztig sur les modules de Verma”, C. R. Acad. Sci. Paris Sér. A-B 291:6 (1980), A373-A376. MR 81k:17004 Zbl 0457.22012

[Brylinski and Kashiwara 1981] J.-L. Brylinski and M. Kashiwara, "Kazhdan-Lusztig conjecture and holonomic systems", Invent. Math. 64:3 (1981), 387-410. MR 83e:22020 Zbl 0473.22009

[Cartier 1979] P. Cartier, "Representations of p-adic groups: A survey", pp. 111-155 in Automorphic forms, representations and L-functions (Corvallis, OR, 1977), edited by A. Borel and W. Casselman, Proc. Sympos. Pure Math. 33, Amer. Math. Soc., Providence, RI, 1979. MR 81e:22029 Zbl 0421.22010

[Chevalley 1955] C. Chevalley, "Sur certains groupes simples", Tôhoku Math. J. (2) 7 (1955), 14-66. MR 17,457c Zbl 0066.01503

[Demazure and Gabriel 1970] M. Demazure and P. Gabriel, Groupes algébriques,I: Géométrie algébrique, généralités, groupes commutatifs, Masson \& Cie, Éditeur, Paris, 1970. MR 46 \#1800 Zbl 0203.23401

[Dixmier 1996] J. Dixmier, Enveloping algebras, Graduate Studies in Mathematics 11, Amer. Math. Soc., Providence, RI, 1996. MR 97c:17010 Zbl 0867.17001 
[Dixon et al. 1999] J. D. Dixon, M. P. F. du Sautoy, A. Mann, and D. Segal, Analytic pro-p groups, 2nd ed., Cambridge Studies in Advanced Mathematics 61, Cambridge University Press, 1999. MR 2000m:20039 Zbl 0934.20001

[Dospinescu and Schraen 2013] G. Dospinescu and B. Schraen, "Endomorphism algebras of admissible $p$-adic representations of p-adic Lie groups", Represent. Theory 17 (2013), 237-246. MR 3053464 Zbl 06183359

[Emerton 2011] M. Emerton, "Locally analytic vectors in representations of locally $p$-adic analytic groups", preprint, 2011, http://www.math.uchicago.edu/ emerton/pdffiles/analytic.pdf. To appear in Mem. Amer. Math. Soc.

[Frommer 2003] H. Frommer, "The locally analytic principal series of split reductive groups", Heft 265 of Preprintreihe SFB 478, Mathematischen Instituts der Westfälischen Wilhelms-Universität Münster, 2003, http://www.math.uni-muenster.de/sfb/about/publ/heft265.ps.

[Godement 1958] R. Godement, Topologie algébrique et théorie des faisceaux, I, Actualités scientifiques et industrielles 1252, Hermann, Paris, 1958. MR 21 \#1583 Zbl 0080.16201

[Grothendieck 1967] A. Grothendieck, "Éléments de géométrie algébrique, IV: Étude locale des schémas et des morphismes de schémas, IV", Inst. Hautes Études Sci. Publ. Math. 32 (1967), 361. MR 39 \#220 Zbl 0153.22301

[Gruson 1966] L. Gruson, "Théorie de Fredholm p-adique", Bull. Soc. Math. France 94 (1966), 67-95. MR 37\#1971 Zbl 0149.34702

[Hotta et al. 2008] R. Hotta, K. Takeuchi, and T. Tanisaki, D-modules, perverse sheaves, and representation theory, Progress in Mathematics 236, Birkhäuser, Boston, 2008. MR 2008k:32022 Zbl 1136.14009

[Huber et al. 2011] A. Huber, G. Kings, and N. Naumann, "Some complements to the Lazard isomorphism”, Compos. Math. 147:1 (2011), 235-262. MR 2012d:22016 Zbl 1268.20051

[Jantzen 2003] J. C. Jantzen, Representations of algebraic groups, 2nd ed., Mathematical Surveys and Monographs 107, Amer. Math. Soc., Providence, RI, 2003. MR 2004h:20061 Zbl 1034.20041

[Kashiwara and Schapira 1990] M. Kashiwara and P. Schapira, Sheaves on manifolds, Grundlehren der Mathematischen Wissenschaften 292, Springer, Berlin, 1990. MR 92a:58132 Zbl 0709.18001

[Kohlhaase 2007] J. Kohlhaase, "Invariant distributions on $p$-adic analytic groups", Duke Math. J. 137:1 (2007), 19-62. MR 2008j:22024 Zbl 1133.11066

[Lazard 1965] M. Lazard, “Groupes analytiques p-adiques”, Inst. Hautes Études Sci. Publ. Math. 26 (1965), 389-603. MR 35 \#188 Zbl 0139.02302

[McConnell and Robson 1987] J. C. McConnell and J. C. Robson, Noncommutative Noetherian rings, Wiley, Chichester, 1987. MR 89j:16023 Zbl 0644.16008

[Miličić 1993a] D. Miličić, "Algebraic $\mathcal{D}$-modules and representation theory of semisimple Lie groups", pp. 133-168 in The Penrose transform and analytic cohomology in representation theory (South Hadley, MA, 1992), edited by M. Eastwood et al., Contemp. Math. 154, Amer. Math. Soc., Providence, RI, 1993. MR 94i:22035 Zbl 0821.22005

[Miličić 1993b] D. Miličić, "Localization and representation theory of reductive Lie groups", preprint, 1993, http://www.math.utah.edu/ milicic/Eprints/book.pdf.

[Moy and Prasad 1994] A. Moy and G. Prasad, "Unrefined minimal $K$-types for $p$-adic groups", Invent. Math. 116:1-3 (1994), 393-408. MR 95f:22023 Zbl 0804.22008

[Noot-Huyghe 2009] C. Noot-Huyghe, "Un théorème de Beilinson-Bernstein pour les D-modules arithmétiques", Bull. Soc. Math. France 137:2 (2009), 159-183. MR 2011e:14038 Zbl 1171.14014

[Orlik and Strauch 2010a] S. Orlik and M. Strauch, "On Jordan-Hölder series of some locally analytic representations", preprint, 2010. To appear in J. Amer. Math. Soc. (article electronically published on July 2, 2014). arXiv 1001.0323 
[Orlik and Strauch 2010b] S. Orlik and M. Strauch, "On the irreducibility of locally analytic principal series representations”, Represent. Theory 14 (2010), 713-746. MR 2012e:22025 Zbl 1247.22018

[Patel et al. 2013] D. Patel, T. Schmidt, and M. Strauch, " $p$-adic analytic representations and semistable models of flag varieties: the case of GL(2)", preprint, 2013. To appear in Münster J. Math. arXiv 1310.3537

[Rémy et al. 2010] B. Rémy, A. Thuillier, and A. Werner, "Bruhat-Tits theory from Berkovich's point of view, I: Realizations and compactifications of buildings", Ann. Sci. Éc. Norm. Supér. (4) 43:3 (2010), 461-554. MR 2011j:20075 Zbl 1198.51006

[Robert 2000] A. M. Robert, A course in p-adic analysis, Graduate Texts in Mathematics 198, Springer, New York, 2000. MR 2001g:11182 Zbl 0947.11035

[Schmidt 2008] T. Schmidt, "Auslander regularity of $p$-adic distribution algebras", Represent. Theory 12 (2008), 37-57. MR 2009b:22018 Zbl 1142.22010

[Schmidt 2013] T. Schmidt, "On locally analytic Beilinson-Bernstein localization and the canonical dimension”, Math. Z. 275:3-4 (2013), 793-833. MR 3127038 Zbl 06254165

[Schneider 2002] P. Schneider, Nonarchimedean functional analysis, Springer, Berlin, 2002. MR 2003a:46106 Zbl 0998.46044

[Schneider and Stuhler 1997] P. Schneider and U. Stuhler, "Representation theory and sheaves on the Bruhat-Tits building", Inst. Hautes Études Sci. Publ. Math. 85 (1997), 97-191. MR 98m:22023 Zbl 0892.22012

[Schneider and Teitelbaum 2002] P. Schneider and J. Teitelbaum, "Locally analytic distributions and p-adic representation theory, with applications to GL 2 ", J. Amer. Math. Soc. 15:2 (2002), 443-468. MR 2003b:11132 Zbl 1028.11071

[Schneider and Teitelbaum 2003] P. Schneider and J. Teitelbaum, "Algebras of p-adic distributions and admissible representations", Invent. Math. 153:1 (2003), 145-196. MR 2004g:22015 Zbl 1028.11070

[Schneider and Teitelbaum 2005] P. Schneider and J. Teitelbaum, "Duality for admissible locally analytic representations", Represent. Theory 9 (2005), 297-326. MR 2006a:22016 Zbl 1146.22301

[Tits 1979] J. Tits, "Reductive groups over local fields", pp. 29-69 in Automorphic forms, representations and L-functions (Corvallis, OR, 1977), edited by A. Borel and W. Casselman, Proc. Sympos. Pure Math. 33, Amer. Math. Soc., Providence, RI, 1979. MR 80h:20064 Zbl 0415.20035

[Vignéras 1997] M.-F. Vignéras, "Cohomology of sheaves on the building and $R$-representations", Invent. Math. 127:2 (1997), 349-373. MR 98k:20079 Zbl 0872.20042

[Washington 1997] L. C. Washington, Introduction to cyclotomic fields, 2nd ed., Graduate Texts in Mathematics 83, Springer, New York, 1997. MR 97h:11130 Zbl 0966.11047

Communicated by Marie-France Vignéras

Received 2012-11-27 Revised 2014-02-20 Accepted 2014-05-23

patel471@purdue.edu Department of Mathematics, Purdue University, 150 North University Street, West Lafayette, IN 47907, United States

Tobias.Schmidt@math.hu-berlin.de

Institut fuer Mathematik, Humboldt-Universität zu Berlin, Rudower Chaussee 25, D-12489 Berlin, Germany

mstrauch@indiana.edu

Department of Mathematics, Indiana University, Rawles Hall, Bloomington, IN 47405, United States 


\section{Algebra \& Number Theory}

msp.org/ant

\section{EDITORS}

MANAGING EDITOR

Bjorn Poonen

Massachusetts Institute of Technology

Cambridge, USA

\author{
EDITORIAL BOARD CHAIR \\ David Eisenbud \\ University of California \\ Berkeley, USA
}

BOARD OF EDITORS

Georgia Benkart

Dave Benson

Richard E. Borcherds

John H. Coates

J-L. Colliot-Thélène

Brian D. Conrad

Hélène Esnault

Hubert Flenner

Edward Frenkel

Andrew Granville

Joseph Gubeladze

Roger Heath-Brown

Craig Huneke

Yujiro Kawamata

János Kollár

Yuri Manin

Barry Mazur

Philippe Michel

Susan Montgomery
University of Wisconsin, Madison, USA

University of Aberdeen, Scotland

University of California, Berkeley, USA

University of Cambridge, UK

CNRS, Université Paris-Sud, France

University of Michigan, USA

Freie Universität Berlin, Germany

Ruhr-Universität, Germany

University of California, Berkeley, USA

Université de Montréal, Canada

San Francisco State University, USA

Oxford University, UK

University of Virginia, USA

University of Tokyo, Japan

Princeton University, USA

Northwestern University, USA

Harvard University, USA

École Polytechnique Fédérale de Lausanne

University of Southern California, USA
Shigefumi Mori

Raman Parimala

Jonathan Pila

Anand Pillay

Victor Reiner

Peter Sarnak

Joseph H. Silverman

Michael Singer

Vasudevan Srinivas

J. Toby Stafford

Bernd Sturmfels

Richard Taylor

Ravi Vakil

Michel van den Bergh

Marie-France Vignéras

Kei-Ichi Watanabe

Efim Zelmanov

Shou-Wu Zhang
RIMS, Kyoto University, Japan

Emory University, USA

University of Oxford, UK

University of Notre Dame, USA

University of Minnesota, USA

Princeton University, USA

Brown University, USA

North Carolina State University, USA

Tata Inst. of Fund. Research, India

University of Michigan, USA

University of California, Berkeley, USA

Harvard University, USA

Stanford University, USA

Hasselt University, Belgium

Université Paris VII, France

Nihon University, Japan

University of California, San Diego, USA

Princeton University, USA

\section{PRODUCTION}

production@msp.org

Silvio Levy, Scientific Editor

See inside back cover or msp.org/ant for submission instructions.

The subscription price for 2014 is US $\$ 225 /$ year for the electronic version, and $\$ 400 /$ year ( $\$ 55$, if shipping outside the US) for print and electronic. Subscriptions, requests for back issues and changes of subscribers address should be sent to MSP.

Algebra \& Number Theory (ISSN 1944-7833 electronic, 1937-0652 printed) at Mathematical Sciences Publishers, 798 Evans Hall \#3840, c/o University of California, Berkeley, CA 94720-3840 is published continuously online. Periodical rate postage paid at Berkeley, CA 94704, and additional mailing offices.

ANT peer review and production are managed by EditFLOW ${ }^{\circledR}$ from MSP.

\section{PUBLISHED BY}

- mathematical sciences publishers

nonprofit scientific publishing

http://msp.org/

(C) 2014 Mathematical Sciences Publishers 


\section{Algebra \& Number Theory}

Volume $8 \quad$ No. $6 \quad 2014$

Decompositions of commutative monoid congruences and binomial ideals

ThOMAS KAHLE and EZRA MiLLER

Locally analytic representations and sheaves on the Bruhat-Tits building

DeEPam Patel, Tobias Schmidt and Matthias Strauch

Complétés universels de représentations de $\mathrm{GL}_{2}\left(\mathbb{Q}_{p}\right)$

Pierre Colmez and Gabriel Dospinescu

On moduli spaces for quasitilted algebras

GRZEGORZ BOBIŃSKI 